\title{
Um método de Lagrangianos aumentados e sua aplicação em otimização de malhas
}

\author{
Ana Paula Mazzini
}





\title{
Um método de Lagrangianos aumentados e sua aplicação em otimização de malhas ${ }^{1}$
}

\author{
Ana Paula Mazzini
}

Orientadora: Profa. Dra. Marina Andretta

Dissertação apresentada ao Instituto de Ciências Matemáticas e de Computação - ICMC-USP, como parte dos requisitos para obtenção do título de Mestre em Ciências - Ciências de Computação e Matemática Computacional. VERSÃO REVISADA

USP - São Carlos

Março de 2012

\footnotetext{
${ }^{1}$ Este trabalho foi financiado pelo $\mathrm{CNPq}$.
} 
Ficha catalográfica elaborada pela Biblioteca Prof. Achille Bassi e Seção Técnica de Informática, ICMC/USP, com os dados fornecidos pelo(a) autor(a)

Mazzini, Ana Paula

M477m Um método de Lagrangianos aumentados e sua aplicação em otimização de malhas / Ana Paula Mazzini; orientadora Marina Andretta. -- São Carlos, 2012.

$95 \mathrm{p}$.

Dissertação (Mestrado - Programa de Pós-Graduação en Ciências de Computação e Matemática Computacional) - Instituto de Ciências Matemáticas e de Computação, Universidade de São Paulo, 2012.

1. Lagrangianos aumentados. 2. Programação nãolinear. 3. Otimização de malhas. I. Andretta, Marina, orient. II. Título. 
Aos meus pais Adair e Marlete e a meus irmãos Carlos e Natalia 


\section{Agradecimentos}

Agradeço primeiramente a Deus, que sempre atendeu minhas preces e acalmou meu coração.

Agradeço aos meus pais, Marlete e Adair, pelo amor, carinho, dedicação, compreensão e apoio, sem os quais eu não estaria concluindo mais esta etapa de minha vida. Agradeço a meu irmão Carlos e minha irmã Natalia, sinto falta de vocês.

Agradeço também aos meus familiares, principalmente à minha querida madrinha Maria, e ao meu querido primo Vagner. Todos vocês formam os pilares dessa minha caminhada.

Agradeço a meu orientador de iniciação científica Prof. Dr. Antônio Roberto Balbo, pela amizade, pelo incentivo e por todo o apoio nesta longa caminhada.

Agradeço especialmente a minha orientadora Profa. Dra. Marina Andretta, pela confiança e por todas as horas dedicadas a me guiar. Agradeço também aos professores Luis Gustavo Nonato, Mário A. S. Liziér e Marcelo F. Siqueira pelas grandiosas contribuições neste trabalho.

Agradeço aos meus amigos, colegas e professores do LOT, pela acolhida em São Carlos, pela ajuda, pelos conselhos, pelas horas de confraternização, em especial à minha amiga Gabriela, por todas as horas que esteve ao meu lado, me aconselhando, me ajudando e me fazendo rir.

Agradeço também aos meus amigos do "Hand", por todas as horas, sendo diversão ou não. Afinal o "Hand rende".

Agradeço também as minhas amigas de longe, que quase não vejo, mas que sempre estão presentes. Geisa, Fernanda e Gisele.

Muitos nomes faltaram. E, a todos, que não citei, muito obrigada! 

Métodos de Lagrangianos aumentados são muito utilizados para resolver problemas de minimização de funções sujeitas a restrições gerais. Em particular, estudamos um método de Lagrangianos aumentados que utiliza a função PHR, implementado em ALGENCAN, e observamos seu comportamento quando o aplicamos na resolução de um problema encontrado na área de Computação Gráfica.

O problema estudado é um problema encontrado na geração de malhas de superfícies, na etapa de pós-processamento, para o qual propomos uma técnica de otimização visando a melhoria dos elementos da malha. Quando se trata de geração de malhas de superfícies em $\mathbb{R}^{3}$, parametrizações de malhas triângulares que representam superfícies são usadas em muitas aplicações de processamento de malhas para vários fins. Muitas vezes é necessário preservar a métrica da superfície e, assim, minimizar a deformação do ângulo e da área. A técnica que propomos de otimização visa melhorar as distorções de ângulos e áreas impostas por uma parametrização.

Para verificar o comportamento da técnica proposta, implementamo-na em C++ e utilizamos algumas malhas de modelos clássicos da literatura para realizar os experimentos numéricos. Os resultados obtidos foram promissores. 

Augmented Lagrangian methods are frequently used to solve minimization problems subject to general constraints. In particular, we study an augmented Lagrangian method that uses the PHR function, implemented in ALGENCAN, and observe its behavior when applied to solve a problem found in the field of Computer Graphics.

The problem we will study and solve is found in the post-processing stage of the surface mesh generation, for which we propose an optimization technique to improve the mesh elements. When it comes to meshing surfaces in $\mathbb{R}^{3}$, triangular meshes parametrizations are widely used in applications of mesh processing. It is often necessary to preserve the surface metric and, thus, minimize the angle and area deformation. The optimization technique we propose aims to improve the distortions imposed by a parametrization onto angles and areas.

To assert the efectiveness of the proposed technique, we implemented it in $\mathrm{C}++$ language and used some classic mesh models from the literature to performe numerical experiments. The results were promising. 

1 Lagrangianos aumentados 3

1.1 Lagrangianos aumentados com formulação PHR . . . . . . . . . . . . . 3

1.1.1 Algoritmo interno . . . . . . . . . . . . . 7

2 Otimização de malhas 13

2.1 Malhas . . . . . . . . . . . . . . . . . . . . 13

2.2 Otimização de malhas . . . . . . . . . . . . . . . . . . . . . . . . 16

2.2.1 Modelo de otimização não-linear . . . . . . . . . . . . . . . . . . . 18

3 Implementação $\mathbf{2 5}$

3.1 Estrutura de dados . . . . . . . . . . . . . . . . . . . 25

3.1 .1 Estrutura de dados da malha grossa . . . . . . . . . . . 26

3.1 .2 Estrutura de dados da malha fina . . . . . . . . . . . . . . 27

3.2 Arquivos de entrada e saída . . . . . . . . . . . . . . . . . 28

3.3 Método para otimização de malhas . . . . . . . . . . . . . . . . . 35

4 Resultados numéricos 39

4.1 Modelos de malhas utilizadas . . . . . . . . . . . . . . . . . . . 39

4.2 Experimentos computacionais . . . . . . . . . . . . . . . 39

4.3 Critério de qualificação . . . . . . . . . . . . . . . . . . . . . 41

4.3 .1 Distorção angular . . . . . . . . . . . . . . . . 41

4.3 .2 Bimba Con Nastrino . . . . . . . . . . . . . . . 41

4.3 .3 Botijo ............................ 41

4.3 .4 Distorção da área . . . . . . . . . . . . . . . . . . . . 45

4.3.5 Bimba Con Nastrino . . . . . . . . . . . . . . . . . 45

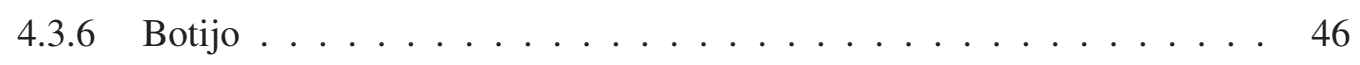

4.4 Consequências da otimização na malha grossa . . . . . . . . . . . . . . . 46 
4.4 .1 Bimba Con Nastrino . . . . . . . . . . . . . . . . . 46

4.4 Botijo ............................... 49

5 Conclusões e trabalhos futuros 53

$\begin{array}{lll}\text { A Algoritmos } & 55\end{array}$

B Superfícies e parametrizações $\quad 57$

B.1 Superfícies . . . . . . . . . . . . . . . . . . . 57

B.2 Primeira Forma Fundamental . . . . . . . . . . . . . . . . . . . . . 59

B.2.1 Comprimento . . . . . . . . . . . . . . . . 62

B.2.2 Ângulo . . . . . . . . . . . . . . . . . . . . 62

B.2.3 Área ........................... 63

B.3 Mapas conformais . . . . . . . . . . . . . . . . . . . . 64

B.4 Mapas equiareais . . . . . . . . . . . . . . . . . . . 66

B.5 Mapas isométricos . . . . . . . . . . . . . . . . . . 67

B.6 Distorções métricas . . . . . . . . . . . . . . . . . . . . . . . . 67

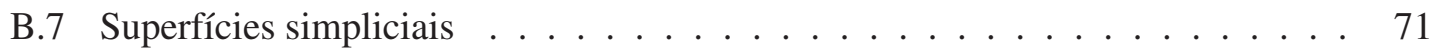

B.8 Mudança de variáveis . . . . . . . . . . . . . . . . . . . . . . . . . . . . . . . 74

C Demonstrações dos teoremas do Apêndice B 79 


\section{Introdução}

Estamos interessados em problemas práticos que podem ser modelados na forma

$$
\begin{array}{ll}
\text { Minimizar } & f(x) \\
\text { sujeito a } & x \in \Omega_{1} \cap \Omega_{2},
\end{array}
$$

onde subproblemas da forma

$$
\begin{array}{ll}
\text { Minimizar } & F(x) \\
\text { sujeito a } & x \in \Omega_{2},
\end{array}
$$

são mais simples de serem resolvidos do que o problema (1), ou seja, são conhecidos algoritmos eficientes para resolvê-los.

Métodos de Lagrangianos aumentados são ferramentas muito populares e eficientes para resolver problemas de programação não-linear escritos na forma (1). Em cada iteração desses métodos, um problema de otimização da forma (2) mais simples é resolvido, para o qual existem algoritmos eficientes.

Em (Birgin (2005)), é realizada uma comparação numérica entre muitos destes métodos de Lagrangianos aumentados, utilizando os problemas adequados da coleção CUTE (Constrained and Unconstrained Testing Environment, Bongartz (1995)) e, como resultado, tem-se que o método de Lagrangianos aumentados usando a formulação PHR, proposta em (Powell (1969); Hestenes (1969); Rockafellar (1973)), é superior em diversas situações.

(Andreani (2007)) introduz um método de Lagrangianos aumentados usando a formulação PHR que não utiliza variáveis de folga e pode ter restrições arbitrárias no conjunto de restrições $\Omega_{2}$. Este método foi implementado em ALGENCAN (Andreani (2007)), está disponível pelo projeto TANGO (TANGO (2005)), e considera $\Omega_{2}$ como um conjunto de restrições de caixa.

Estudamos o método de Lagrangianos aumentados implementado em ALGENCAN e observamos seu comportamento quando aplicamos a um problema encontrado na área de Computação Gráfica. 
A Computação Gráfica é a área da computação designada à geração de imagens a partir de um conjunto de dados. Este dados podem ser gerados de forma interativa ou por modelos que simulem fenômenos reais. Possui uma infinidade de aplicações para diversas áreas.

O problema estudado é um problema encontrado na geração de malhas de superfícies, na etapa de pós-processamento, para o qual propomos uma técnica de otimização visando a melhoria dos elementos da malha.

Construir uma malha não é simplesmente uma questão de criar uma malha do domínio de interesse, mas a obtenção de uma malha de boa qualidade. Existem algumas técnicas de otimização para melhorar a qualidade de malhas de superfícies. Em (Degener (2003)) é proposta uma fórmula de energia que mede simultaneamente as deformações de ângulo e área impostas por uma parametrização.

Quando se trata de geração de malhas de superfícies em $\mathbb{R}^{3}$, parametrizações de malhas triângulares que representam superfícies são usadas em muitas aplicações de processamento de malhas para vários fins. Muitas vezes é necessário preservar a métrica da superfície e, assim, minimizar a deformação do ângulo e da área. A técnica de otimização que propomos visa melhorar as distorções de ângulos e áreas impostas por uma parametrização.

Procuramos, dada uma parametrização conhecida, mover a malha grossa de maneira que minimizemos as distorções de área e ângulo impostas por esta parametrização.

Utilizamos dois modelos de uma mesma superfície para descrever o processo de otimização, o que chamamos de malha grossa e malha fina e todo o processo de otimização que vamos apresentar ocorrerá nas coordenadas bidimensionais das estrelas da malha fina contidas em cada uma das estrelas de cada vértice da malha grossa.

No Capítulo 1, apresentamos um estudo sobre Lagrangianos aumentados e a formulação PHR, que utilizamos para otimização de malhas de superfícies. No Capítulo 2, descrevemos as principais características sobre malhas e a modelagem do problema de otimização não-linear que resolvemos. No Capítulo 3, descrevemos detalhes de implementação do método de otimização proposto, que utiliza ALGENCAN. No Capítulo 4 descrevemos os resultados numéricos obtidos quando utilizamos uma implementação do método proposto para a resolução do problema de otimização em malhas de modelos clássicos da literatura. No Capítulo 5, apresentamos as conclusões deste trabalho e propostas para trabalhos futuros. 


7

\section{Lagrangianos aumentados}

Neste capítulo vamos descrever um método de Lagrangianos aumentados, implementado em AlgENCAN (Andreani (2007)), suas principais características e quais tipos de problema podemos resolver com este método.

\subsection{Lagrangianos aumentados com formulação PHR}

Um problema de programação não-linear pode ser modelado como a minimização (ou maximização) de uma função não-linear sujeita a restrições arbitrárias.

Estamos interessados em problemas práticos que podem ser modelados na forma

$$
\begin{array}{ll}
\text { Minimizar } & f(x) \\
\text { sujeito a } & x \in \Omega_{1} \cap \Omega_{2},
\end{array}
$$

onde subproblemas da forma

$$
\begin{array}{ll}
\text { Minimizar } & F(x) \\
\text { sujeito a } & x \in \Omega_{2},
\end{array}
$$

são mais simples de serem resolvidos do que o problema (1.1), ou seja, são conhecidos algoritmos eficientes para resolvê-los.

Para o caso mais geral, considere o problema de programação não-linear 


$$
\begin{array}{ll}
\text { Minimizar } & f(x) \\
\text { sujeito a } & h_{1}(x)=0 \\
& g_{1}(x) \leq 0 \\
& h_{2}(x)=0 \\
& g_{2}(x) \leq 0
\end{array}
$$

onde $h_{1}: \mathbb{R}^{n} \rightarrow \mathbb{R}^{m_{1}}, g_{1}: \mathbb{R}^{n} \rightarrow \mathbb{R}^{p_{1}}, h_{2}: \mathbb{R}^{n} \rightarrow \mathbb{R}^{m_{2}}, g_{2}: \mathbb{R}^{n} \rightarrow \mathbb{R}^{p_{2}}$ e $f: \mathbb{R}^{n} \rightarrow \mathbb{R}$ são suaves.

Definimos $\Omega_{1}=\left\{x \in \mathbb{R}^{n} \mid h_{1}(x)=0\right.$ e $\left.g_{1}(x) \leq 0\right\}$ e $\Omega_{2}=\left\{x \in \mathbb{R}^{n} \mid h_{2}(x)=0\right.$ e $\left.g_{2}(x) \leq 0\right\}$. Métodos de Lagrangianos aumentados são muito populares e eficientes para resolver este problema usando a formulação (1.2), sendo sua função mais conhecida dada pela fórmula PHR (Powell (1969); Hestenes (1969); Rockafellar (1973)):

$$
\mathcal{L}_{\rho}(x, \chi, \mu)=f(x)+\frac{\rho}{2}\left\{\sum_{i=1}^{m_{1}}\left[h_{1 i}(x)+\frac{\chi_{i}}{\rho}\right]^{2}+\sum_{i=1}^{p_{1}}\left[\max \left(0, g_{1 i}(x)+\frac{\mu_{i}}{\rho}\right)\right]^{2}\right\}
$$

para todo $x \in \mathbb{R}^{n}, \chi \in \mathbb{R}^{m_{1}}, \mu \in \mathbb{R}_{+}^{p_{1}}, \rho>0$.

Métodos de Lagrangianos aumentados que usam tal formulação são baseados na minimização iterativa de $\mathcal{L}_{\rho}$ respeitando $\Omega_{2}$, seguida da atualização do parâmetro de penalidade $\rho$ e dos multiplicadores de Lagrange $\chi$ e $\mu$.

(Andreani (2007)) introduz um método de Lagrangiano aumentado usando a formulação PHR que pode ter restrições arbitrárias no conjunto $\Omega_{2}$. Sua convergência é baseada nas condições CPLD (Constant Positive Linear Dependence) e a limitação dos parâmetros de penalidade foram demonstrados em (Andreani (2007)).

A condição CPLD para os pontos possíveis de problemas de programação não-lineares foi introduzida em (Qi \& Wei (2000)) e utilizado para a análise dos métodos de programação quadrática seqüencial (SQP). No artigo onde o CPLD foi introduzida, os autores presumiram que esta condição poderia ser uma restrição de qualificação. Em (Andreani (2004)) chega-se a conclusão de que CPLD é realmente um critério de qualificação válido.

A forma geral do método de Lagrangiano aumentado baseado na formulação PHR é apresentada pelo Algoritmo 1 .

Na implementação deste algoritmo, são calculados $\chi_{i}^{k+1}=\min \left\{\max \left\{\chi_{\min }, \chi_{i}^{k}+\rho_{k} h_{1_{i}}\left(x_{k}\right)\right\}\right.$, $\left.\chi_{\max }\right\}$ e $\mu_{i}^{k+1}=\min \left\{\max \left\{0, \mu_{i}^{k}+\rho_{k} g_{1_{i}}\left(x_{k}\right)\right\}, \mu_{\max }\right\}$ como definidos em (Hestenes (1969)), sendo estas as estimativas de primeira ordem dos multiplicadores de Lagrange com salvaguardas. As salvaguardas definidas por $\chi_{\min }, \chi_{\max }$ e $\mu_{\max }$ são necessárias para mostrar resultados de convergência global, não necessitando de hipóteses fortes sobre o problema para garantir a limitação dos parâmetros de penalidade. Na prática o intervalo $\left[\chi_{\min }, \chi_{\max }\right]$ não pode ser definido, sendo usada uma estimativa. 
Algoritmo 1 Método de Lagrangiano aumentado usando a formulação PHR para resolver (1.3)

Sejam $\chi_{\min }<\chi_{\max }, \mu_{\max }>0, \gamma>1,0<\rho<1$. Seja $\left\{\varepsilon_{k}\right\}$ uma sequência de números não negativos tal que $\lim _{k \rightarrow \infty} \varepsilon_{k}=0$. Sejam $\chi_{i}^{1} \in\left[\chi_{\min }, \chi_{\max }\right], i=1, \ldots, m_{1}, \mu_{i}^{1} \in\left[0, \mu_{\max }\right]$, $i=1, \ldots, p_{1}$ e $\rho_{1}>0$. Faça $k \leftarrow 1$.

Passo 1. Critério de convergência.

Defina

$$
V_{i}^{k}=\max \left\{g_{i}\left(x_{k}\right),-\frac{\mu_{i}^{k}}{\rho_{k}}\right\}, \quad i=1, \ldots, p
$$

$\mathrm{Se}$

$$
\max \left\{\left\|h_{i}\left(x_{k}\right)\right\|_{\infty},\left\|V_{i}^{k}\right\|_{\infty}\right\} \leq \varepsilon_{k}
$$

e

$$
\left\|P_{\Omega_{2}}\left(x_{k}-\nabla \mathcal{L}_{\rho_{k}}\left(x_{k}, \chi^{k}, \mu^{k}\right)\right)-x_{k}\right\| \leq \varepsilon_{k}
$$

então PARE e devolva $x_{k-1}$ como solução.

Passo 2. Resolução do subproblema.

Calcule $x_{k} \in \mathbb{R}^{n}$ uma solução aproximada de

$$
\text { Minimizar } \quad \mathcal{L}_{\rho_{k}}\left(x_{k}, \chi^{k}, \mu^{k}\right) \quad \text { sujeito a } \quad x_{k} \in \Omega_{2} \text {. }
$$

Passo 3. Atualização do parâmetro de penalidade.

Defina

$$
V_{i}^{k}=\max \left\{g_{1_{i}}\left(x_{k}\right),-\frac{\mu_{i}^{k}}{\rho_{k}}\right\}, \quad i=1, \ldots, p_{1} .
$$

Se $k=1$ ou $\max \left\{\left\|h_{1}\left(x_{k}\right)\right\|_{\infty},\left\|V_{i}^{k}\right\| \infty\right\} \leq \tau \max \left\{\left\|h_{1}\left(x_{k-1}\right)\right\|_{\infty},\left\|V_{i}^{k-1}\right\|_{\infty}\right\}$ então defina $\rho_{k+1}=\rho_{k}$.

Senão, defina $\rho_{k+1}=\gamma \rho_{k}$.

Passo 4. Atualização dos multiplicadores de Lagrange.

Calcule $\chi_{i}^{k+1} \in\left[\chi_{\min }, \chi_{\max }\right], i=1, \ldots, m_{1}$, e $\mu_{i}^{k_{1}} \in\left[0, \mu_{\max }\right], i=1, \ldots, p_{1}$. Faça $k \leftarrow k+1$ e volte ao Passo 1; 
Supondo que $f, g$ e $h$ admitem primeira derivada contínua, precisamos de uma definição precisa para a solução aproximada de (1.4). Isto é feito no Algoritmo 2, que é definido como o Algoritmo 1, trocando apenas o Passo 2.

Algoritmo 2 Algoritmo 1 trocando apenas o Passo 2

Passo 2. Resolução do subproblema.

Calcule (se possível) $x_{k} \in \mathbb{R}^{n}$ tal que existam $v^{k} \in \mathbb{R}^{m_{2}}$ e $u^{k} \in \mathbb{R}^{p_{2}}$ que satisfaçam

$$
\begin{aligned}
& \left\|\mathcal{L}_{\rho_{k}}\left(x_{k}, \chi^{k}, \mu^{k}\right)+\sum_{i=1}^{m_{2}} v^{k} \nabla h_{2_{i}}\left(x_{k}\right)+\sum_{i=1}^{p_{2}} u^{k} \nabla g_{2_{i}}\left(x_{k}\right)\right\| \leq \varepsilon_{k}, \\
& u_{i}^{k} \geq 0, \quad g_{2_{i}}\left(x_{k}\right) \leq \varepsilon_{k}, \quad \forall i=1, \ldots, p_{2}, \\
& g_{2_{i}}\left(x_{k}\right)<-\varepsilon_{k} \Rightarrow u_{i}^{k}=0, \quad \forall i=1, \ldots, p_{2}, \quad\left\|h_{2}\left(x_{k}\right)\right\| \leq \varepsilon_{k} .
\end{aligned}
$$

A seguir temos os principais teoremas de convergência para o Algoritmo 2 demonstrados em (Andreani (2007)).

Teorema 1.1. Seja $\left\{x_{k}\right\}$ a sequência gerada pelo Algoritmo 2. Suponha que $x^{*}$ seja um ponto limite de $\left\{x_{k}\right\}$. Então, se a sequência de parâmetros de penalidade $\left\{\rho_{k}\right\}$ for limitada, o ponto limite $x^{*}$ é viável. Caso contrário, vale ao menos uma das seguintes possibilidades:

1. $x^{*}$ é um ponto KKT do problema

$$
\begin{array}{ll}
\text { Minimizar } & \frac{1}{2}\left[\sum_{i=1}^{m_{1}}\left[h_{1}(x)\right]_{i}^{2}+\sum_{i=1}^{p_{1}} \max \left\{0,\left[g_{1}(x)_{i}^{2}\right]\right\}\right] \\
\text { sujeito a } & x \in \Omega_{2}
\end{array}
$$

2. $x^{*}$ não satisfaz a condição de qualificação de restrição CPLD associada a $\Omega_{2}$.

Teorema 1.2. Seja $\left\{x_{K}\right\}$ a sequência gerada pelo Algoritmo 2. Suponha que $x^{*}$ seja um ponto limite viável do problema (1.3) que satisfaz a condição de qualificação de restrição CPLD relacionada ao conjunto de todas as retrições. Então, $x^{*}$ é um ponto KKT do problema (1.3).

Em (Andreani (2007)), encontra-se também a demonstração de que a sequência de parâmetros de penalidade $\left\{\rho_{k}\right\}$ é limitada. Interessa-nos o caso em que $\Omega_{2}$ é um conjunto de restrições de caixa, ou seja, $\Omega_{2}=\left\{x \in \mathbb{R}^{n} \mid \ell \leq x \leq u\right\}$. Por continuidade da função Lagrangiano aumentado e compacidade de $\Omega_{2}$, esta definição garante que existe um minimizador global. Para resolver este problema, reescrevemos o Algoritmo 1 trocando apenas o Passo 2, gerando o Algoritmo 3. 
Algoritmo 3 Algoritmo 1 trocando apenas o Passo 2

Passo 2. Resolução do subproblema.

Calcule $x_{k} \in \mathbb{R}^{n}$ tal que

$$
x_{k} \in \Omega_{2} \quad \text { e } \quad\left\|P_{\Omega_{2}}\left(x_{k}-\nabla \mathcal{L}_{\rho_{k}}\left(x_{k}, \chi^{k}, \mu^{k}\right)\right)-x_{k}\right\| \leq \varepsilon_{k},
$$

onde $P_{\Omega_{2}}(\cdot)$ é a projeção ortogonal em $\Omega_{2}$.

É simples verificar que a condição (1.6) é equivalente as condições (1.5). Os Teoremas 1.1 e 1.2 obviamente se aplicam ao Algoritmo 3. Como todos os pontos de $\Omega_{2}$ satisfazem CPLD, o Teorema 1.1 garante que os pontos limite da sequência gerada $\left\{x_{k}\right\}$ são pontos KKT do problema

$$
\begin{array}{ll}
\text { Minimizar } & \frac{1}{2}\left[\sum_{i=1}^{m_{1}}\left[h_{1}(x)\right]_{i}^{2}+\sum_{i=1}^{p_{1}} \max \left\{0,\left[g_{1}(x)_{i}^{2}\right]\right\}\right] \\
\text { sujeito a } & x \in \Omega_{2}
\end{array}
$$

AlgEnCAN é uma implementação do Algoritmo 3, que faz parte do projeto TANGO (TANGO (2005)), que utiliza GENCAN (Birgin \& Martínez (2002)) ou BETRA (Andretta (2005)) para resolver o Passo 2. GENCAN é um método de restrições ativas de minimização de problemas de grande porte com restrições de caixa, que combina um método irrestrito com Gradiente Espectral Projetado (SPG, Birgin $(2005,2000)$ ) para eliminar restrições do conjunto de variáveis livres. O algoritmo irrestrito inclui uma busca linear e um método do tipo Newton truncado. BETRA é um método de restrições ativas para problemas de minimização de pequeno porte em caixas, onde o algoritmo interno é um método de regiões de confiança euclidiano clássico e as iterações para deixar as faces são do tipo SPG, que também é utilizado quando algumas restrições inativas são "quase" ativas.

AlGENCAN tem sido muito utilizado e constantemente melhorado. Por se tratar de um método de Lagrangianos aumentados, a incorporação de novos algoritmos internos para resolver o Passo 2 é quase imediata.

Na Seção 1.1.1 apresentamos os algoritmos GENCAN e BETRA.

\subsubsection{Algoritmo interno}

O subproblema a ser resolvido em cada iteração do método de Lagrangiano aumentado é

$$
\begin{array}{ll}
\text { Minimizar } & \bar{f}(x) \\
\text { sujeito a } & x \in \Omega,
\end{array}
$$

onde $\Omega=\left\{x \in \mathbb{R}^{n} \mid \ell \leq x \leq u\right\}, \ell, u \in \mathbb{R}^{n}, \ell \leq u$ e $\bar{f}(x): \mathbb{R}^{n} \rightarrow \mathbb{R} \subset \mathcal{C}^{2}$.

Para resolver este problema, tanto BETRA como GENCAN utilizam um método de restrições ativas. Sabendo qual é o conjunto de restrições ativas na solução, o ponto $x^{*}$ solução do 
problema é facilmente calculado, já que o problema se torna "irrestrito". Métodos de restrições ativas tentam, a cada iteração, descobrir qual o conjunto de restrições ativas na solução. Se detectam que o conjunto $\mathrm{W}$ de restrições que supunham ser ativas na solução está incorreto, utilizam algum critério para acrescentar ou remover restrições de W.

Em (Andretta (2005); Birgin \& Martínez (2002)), a região viável $\Omega$ é dividida em faces abertas disjuntas. Para todo $I \subset\{1,2, \ldots, n, n+1, n+2, \ldots, 2 n\}$ definimos

$$
F_{I}=\left\{x \in \Omega \mid x_{i}=\ell_{i} \text { se } i \in I, x_{i}=u_{i} \text { se } n+1 \in I, \ell_{i}<x_{i}<u_{i} \text { caso contrário }\right\} .
$$

O conjunto $\Omega$ é a união das faces abertas. Definimos $V_{I}$ o menor espaço afim que contém $F_{I}$ e $S_{I}$ o subespaço linear paralelo a $V_{I}$. O gradiente projetado contínuo é dado por

$$
g_{P}(x)=P_{\Omega}(x-\nabla f(x))-x
$$

e, para todo $x \in F_{I}$, o gradiente interno é dado por

$$
g_{I}(x)=P_{S_{I}}\left[g_{P}(x)\right] .
$$

No método de restrições ativas implementado em BETRA e GENCAN, o conjunto viável é dividido em faces abertas e, a cada iteração $k$, tem-se um ponto $x_{k}$ pertencente a uma dessas faces. No início de cada iteração, verifica-se qual a relação entre a norma do gradiente interno e a norma do gradiente projetado contínuo. Se a norma do gradiente projetado contínuo é "muito maior" que a norma do gradiente interno, decide-se mudar de face e utiliza-se uma iteração do método SPG. Em SPG, na iteração $k$ é calculada uma direção de descida $d^{k}$ e feita uma busca linear para que $x^{k}+t_{k} d^{k}, t_{k}>0$, satisfaça a condição de Armijo, definindo assim o novo ponto $x_{k+1}$, pertencente a uma nova face aberta. Se a norma do gradiente projetado contínuo não é "muito maior" do que a norma do gradiente interno, decide-se continuar na mesma face e utiliza-se uma iteração de um algoritmo "irrestrito" para obter um ponto $x_{k+1}$ no fecho da face atual, no qual a função tem um valor menor do que em $x_{k}$. Este algoritmo "irrestrito" deve ter o cuidado de manter o ponto $x_{k+1}$ viável.

No Passo 2 do Algoritmo 4, quando decide-se permanecer na face atual, utiliza-se um algoritmo "irrestrito". Em BETRA, utiliza-se um método de regiões de confiança com o algoritmo Moré-Sorensen (Moré \& Sorensen (1983)) para minimização exata de quadráticas em bolas a fim de resolver cada subproblema. No Algoritmo 5 temos uma iteração do algoritmo "irrestrito" utilizado em BETRA.

No Passo 3, $p_{k}$ é calculado usando o algoritmo Moré-Sorensem para minimização exata de quadrática em bolas (usando o parâmetro $\sigma$ ). Desta forma, obtém-se um ponto $p_{k}$ tal que $\left\|x_{k}+p_{k}\right\| \leq(1+\sigma) \Delta_{k}$. Assim, quando $\Delta_{k}$ é recalculado no Passo 5, o próximo ponto $x_{k}+p_{k}$ necessariamente pertence ao conjunto viável. Mais detalhes podem ser vistos em (Andretta (2005)). 
Algoritmo 4 Método de restrições ativas implementado em BETRA e GENCAN

$\operatorname{Dados} x_{0} \in \Omega$ e $\eta \in(0,1)$. Faça $k \leftarrow 0$.

Passo 1. Critério de Convergência

Se $g_{P}\left(x_{k}\right)=0$ então PARE e devolva $x_{k}$ como solução

Passo 2. Decisão de permanecer ou não na mesma face

$\mathrm{Se}$

$$
\left\|g_{I}\left(x_{k}\right)\right\| \geq \eta\left\|g_{P}\left(x_{k}\right)\right\|
$$

então considere o problema (1.7) restrito às variáveis livres e use uma iteração de um algoritmo "irrestrito" para calcular $x_{k+1} \in \bar{F}_{I}$. Se o algoritmo "irrestrito" terminar sua execução com $x_{k}$ declarando "Ponto estacionário de primeira ordem" ou "Ponto estacionário de segunda ordem", PARE e devolva $x_{k}$ como solução, declarando o mesmo que o algoritmo "irrestrito".

Senão, faça uma iteração do SPG para calcular $x_{k+1}$.

Passo 3. Faça $k \leftarrow k+1$ e volte ao Passo 1 .

Em GenCAN, utiliza-se um método de Newton truncado com busca linear. No Algoritmo 6 apresentamos uma iteração do algoritmo "irrestrito" utilizado em GENCAN.

No Passo 2, $p_{k}$ é calculado utilizando um método de gradientes conjugados para resolver o sistema Newtoniano

$$
B_{k} p_{k}=-\nabla \bar{f}\left(x_{k}\right),
$$

onde $B_{k}$ é uma aproximação da Hessiana de $\bar{f}$ no ponto $x_{k}$. A cada iteração de gradientes conjugados, toma-se o cuidado de satisfazer a condição (1.10). Quando está não é valida, toma-se o ponto calculado na iteração anterior. No Passo 4, utiliza-se "backtracking" com interpolação quadrática uni-dimensional para calcular $\alpha_{k}$. Esse algoritmo "backtracking" pode ser encontrado no Apêndice A. Mais detalhes podem ser vistos em (Birgin \& Martínez (2002)).

Em Betra e GenCAN, após calcular $x_{k+1}$, utiliza-se a técnica de extrapolação para o cálculo de um ponto com valor de função melhor do que $x_{k+1}$ e, possivelmente, com mais restrições ativas. $\mathrm{O}$ algoritmo de extrapolação pode ser encontrado no Apêndice A.

A seguir apresentamos os teoremas de convergência dos algoritmos internos, BETRA e GENCAN. Primeiramente, vejamos os algoritmos relacionados ao BETRA.

Teorema 1.3. Seja $A \subset \mathbb{R}^{m}$ um conjunto fechado, limitado e convexo, não vazio. Na execução do Algoritmo Interno, ocorre uma e somente uma das seguintes possibilidades:

1. o algoritmo termina em um número finito de iterações num ponto $x_{k}$ tal que a distância de $x_{k}$ à borda é menor que $2 \Delta_{\min } e \nabla \bar{f}\left(x_{k}\right)=0$; 


\section{Algoritmo 5 Iteração do algoritmo "irrestrito" de BETRA}

Seja $k$ a iteração atual. Seja $F_{I}$ a face atual. Dados $x_{k}$ viável, $\Delta_{\min }>0, \Delta_{k} \geq \Delta_{\min }$ e $\sigma>0$.

Passo 1. Se $\nabla \bar{f}\left(x_{k}\right)=0$ então PARE e devolva $x_{k}$ como solução declarando "Ponto estacionário de primeira ordem".

Passo 2. Calcule $\Delta_{b o r d a}$ a distância de $x_{k}$ à borda da face.

Se $\Delta_{b o r d a}<2 \Delta_{\text {min }}$ então faça uma iteração de SPG restrito a $F_{I}$ para calcular $x_{k+1}$ e PARE.

Passo 3. Calcule $p_{k}$ uma solução aproximada de

$$
\begin{array}{ll}
\text { Minimizar } & \varphi(w) \equiv \frac{1}{2} w^{T} \nabla^{2} \bar{f}\left(x_{k}\right) w+\left(\nabla \bar{f}\left(x_{k}\right)\right)^{T} w \\
\text { sujeito a } & \|w\| \leq \Delta_{k}
\end{array} .
$$

Passo 4. Se $\varphi\left(p_{k}\right)=0$ então PARE e devolva $x_{k}$ como solução declarando "Ponto estacionário de segunda ordem".

Passo 5. Se $x_{k}+p_{k}$ não pertence à face atual calcule

$$
\alpha_{\max }=\max \left\{\alpha \in[0,1] \mid\left[x_{k}, x_{k}+\alpha p_{k}\right] \subset \Omega\right\} .
$$

Se $\bar{f}\left(x_{k}+\alpha_{\max } p_{k}\right)<\bar{f}\left(x_{k}\right)$ então faça $x_{k+1} \leftarrow x_{k}+\alpha p_{k}$ e PARE declarando "Iterando na borda". Senão, faça

$$
\Delta_{k} \leftarrow \Delta_{\min }+0.9\left\{\frac{\Delta_{\text {borda }}}{1+\sigma}-\Delta_{\min }\right\}
$$

e volte ao Passo 3.

Passo 6. Calcule $\rho_{k}=\frac{\bar{f}\left(x_{k}+p_{k}\right)-\bar{f}\left(x_{k}\right)}{\varphi\left(p_{k}\right)}$.

Passo 7. Se $\rho \leq 0.1$ então escolha $\Delta_{k} \leftarrow \Delta \in\left[0.1\left\|p_{k}\right\|, 0.9\left\|p_{k}\right\|\right]$ e volte para o Passo 2 .

Passo 8. Faça $x_{k+1} \leftarrow x_{k}+p_{k}$.

Passo 9. Escolha $\Delta_{k+1} \geq \Delta_{\min }$. 
Algoritmo 6 Iteração do algoritmo "irrestrito" de GENCAN

Seja $k$ a iteração atual. Dados $x_{k}$ viável, $\theta \in(0,1)$, e $\vartheta \in(0,1)$

Passo 1. Se $\nabla \bar{f}\left(x_{k}\right)=0$ então PARE e devolva $x_{k}$ como solução declarando "Ponto estacionário de primeira ordem".

Passo 2. Calcule um vetor não-nulo $p_{k} \in \mathbb{R}^{n}$ que satisfaça

$$
\left(\nabla \bar{f}\left(x_{k}\right)\right)^{T} p_{k} \leq-\theta\left\|\nabla \bar{f}\left(x_{k}\right)\right\|\left\|p_{k}\right\| \text {. }
$$

Passo 3. Calcule $\alpha_{\max }=\max \left\{\alpha \in[0,1] \mid x_{k}+\alpha p_{k} \in \Omega\right\}$.

Se $\alpha_{\max }<1$ e $\bar{f}\left(x_{k}+\alpha_{\max } p_{k}\right)<\bar{f}\left(x_{k}\right)$ então faça $x_{k+1}=x_{k}+\alpha_{\max } p_{k}$ e PARE declarando "Iterando na borda".

Passo 4. Calcule $\alpha_{k} \leq \alpha_{\max }$ para o qual valha $\bar{f}\left(x_{k}+\alpha_{k} p_{k}\right) \leq \bar{f}\left(x_{k}\right)+\vartheta \alpha_{k}\left(\nabla \bar{f}\left(x_{k}\right)\right)^{T} p_{k}$.

Passo 5. Faça $x_{k+1}=x_{k}+\alpha_{k} p_{k}$.

2. o Algoritmo Interno termina em um número finito de iterações num ponto estacionário de segunda ordem $x_{k}$ tal que sua distância $\Delta_{b o r d a} \geq 2 \Delta_{\text {min }}$;

3. o Algoritmo Interno termina depois de um número finito de iterações num ponto $x_{k+1}$ na borda de A e

$$
\bar{f}\left(x_{k+1}\right)<\bar{f}\left(x_{k}\right)<\ldots<\bar{f}\left(x_{0}\right)
$$

4. o Algoritmo Interno gera uma sequência infinita de iterandos. Neste caso: (a) todo ponto limite $x^{*}$ da sequência gerada satisfaz $g_{I}\left(x^{*}\right)=0 ;(b)$ se um ponto limite $x^{*}$ é tal que sua distância até a borda é maior que $2 \Delta_{\min }$ então $\nabla^{2} \bar{f}\left(x^{*}\right)$ é semidefinida positiva.

Teorema 1.4. (Teorema de convergência do BETRA) Vale uma das seguintes afirmações:

1. A execução do BETRA termina numa iteração onde o Algoritmo Interno termina com $g_{p}\left(x_{k}\right)=0$. Neste caso, se $x_{k} \in F_{I}$ e a distância entre $x_{k}$ e a borda de $F_{I}$ é maior que $2 \Delta_{\min }$, a matriz $\nabla^{2} f\left(x_{k}\right)$ é semidefinida positiva quando restrita a $F_{I}$;

2. BETRA gera uma sequência infinita de iterandos e pelo menos um ponto limite dessa sequência é um ponto estacionário de primeira ordem.

No segundo caso, se todos os pontos limite são não-degenerados, todos os iterandos pertencerão, em algum momento, à mesma face aberta $F_{I}$. Neste caso, se a distância de um ponto limite $x^{*}$ à borda de $F_{I}$ for maior que $2 \Delta_{\min }$, então $\nabla^{2} f\left(x^{*}\right)$ é semidefinida positiva quando restrita a $F_{I}$. Mais ainda, se esta matriz reduzida for definida positiva, a sequência converge quadraticamente a $x^{*}$. 
As provas dos Teorema 1.3 e 1.4 pode ser encontradas em (Andretta (2005)). A seguir temos os principais teoremas de convergência para o GENCAN. Podemos encontrar suas provas em (Birgin \& Martínez (2002)).

Teorema 1.5. O algoritmo GENCAN é bem definido.

Teorema 1.6. Suponha que $\left\{x^{k}\right\}$ é gerada pelo Algoritmo GENCAN. Suponha que existe $\bar{k} \in$ $\{0,1,2, \ldots\}$ tal que $x^{k} \in F_{I}$ para todos os $k \geq \bar{k}$. Então, cada ponto limite de $\left\{x_{k}\right\}$ é ponto estacionário de primeira ordem.

Teorema 1.7. Suponha que, para todos os $k \in\{0,1,2, \ldots\}, x^{k} \in F_{I}$, existe $k^{\prime}>k$ tal que $x_{k^{\prime}} \notin F_{I}$. Então, existe um ponto limite de $\left\{x_{k}\right\}$ que é ponto estacionário de primeira ordem.

Teorema 1.8. Suponha que todos os pontos fixos de (1.2) são não-degenerados $\left(\frac{\partial f}{\partial x_{i}}(x)=0\right.$ somente se $\ell_{i} \leq x_{i} \leq u_{i}$ ). Então, a hipótese do Teorema 1.6 (e, portanto, a sua tese) é mantida.

Teorema 1.9. Suponha que $\left\{x_{k}\right\}$ é uma seqüência gerada pelo algoritmo GENCAN e seja $\epsilon$ um número positivo arbitrário. Então, existe $k \in\{0,1,2, \ldots\}$ de tal forma que $\left\|g_{P}\left(x_{k}\right)\right\| \leq \epsilon$.

No próximo capítulo, apresentamos um problema que surge durante a geração de malhas para representar figuras em três dimensões e que pode ser modelado como um problema de programação não-linear. 


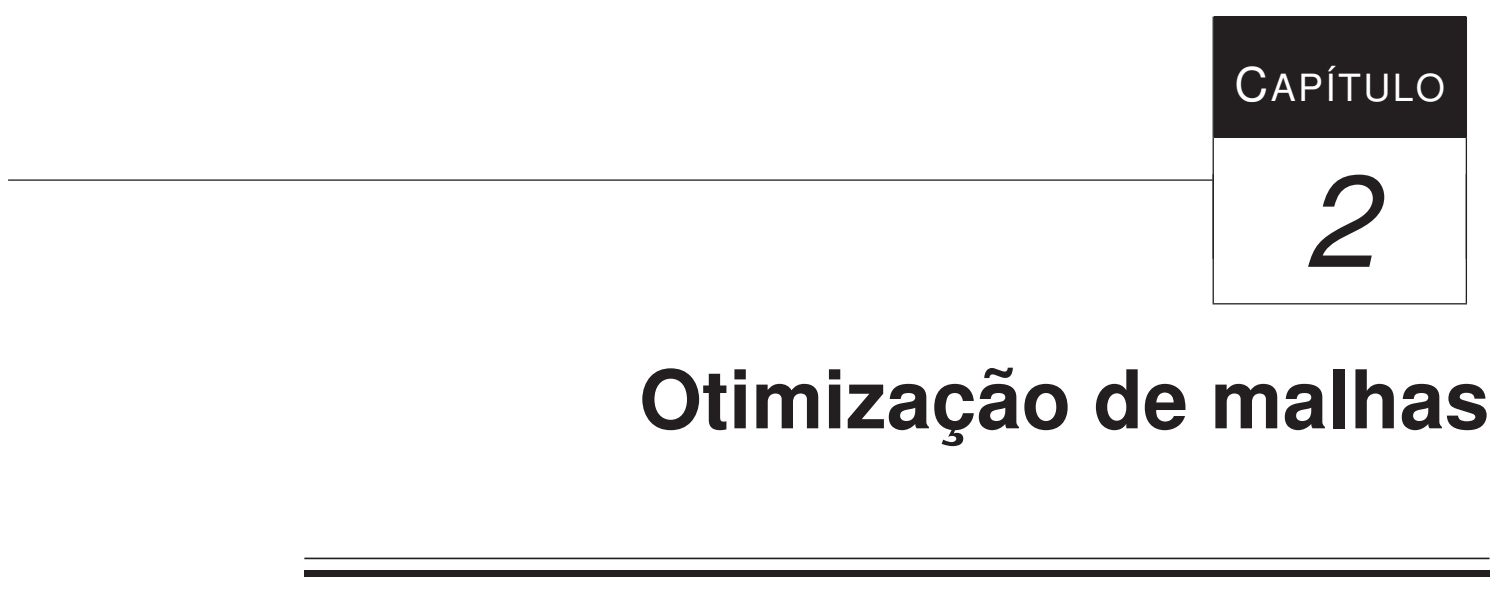

Neste capítulo definimos o que são malhas, que características são importantes e qual modelo de programação não-linear usamos para minimizar uma distorção imposta em uma parametrização de uma malha de superfície em $\mathbb{R}^{3}$ para o plano.

\subsection{Malhas}

As definições 2.1 e 2.2 apresentam uma formalização dos conceitos usados aqui e podem ser encontradas em (Guibas \& Stolfi (1985)) e (Gross \& Tucker (2001)). Considere $\mathbb{E}^{n}$ o espaço Euclidiano afim dotado do produto escalar como produto interno.

Definição 2.1. Uma subdivisão de uma superfície $S \in \mathbb{E}^{n}$ é uma partição $\mathcal{P}$ de $S$ em três coleções finitas de partes disjuntas: vértices, arestas e faces, que denotamos por $V_{S}(\mathcal{P}), E_{S}(\mathcal{P})$ e $F_{S}(\mathcal{P})$, respectivamente, e que satisfazem as condições enumeradas a seguir:

1. Todo vértice é um ponto de $S$.

2. Toda aresta é uma curva em $S$.

3. Toda face é um subconjunto de $S$ homeomorfo ao disco unitário aberto $\mathbb{B}^{2}$ em $\mathbb{E}^{2}$.

4. A fronteira de toda face é um caminho fechado em $S$ formado por vértices e arestas.

Os vértices, arestas e faces de uma subdivisão são chamados de elementos da subdivisão. 
Definição 2.2. Uma triangulação de uma superfície $S \subset \mathbb{E}^{n}$ é uma subdivisão de $S$ na qual a fronteira de cada face consiste de exatamente três arestas e três vértices distintos.

O termo malha é usado para representar triangulações, quadrilaterizações, entre outros, que são casos particulares e importantes de subdivisão de um objeto. Tais subdivisões nos permitem obter informações locais sobre a topologia e a geometria de um objeto. Estamos interessados, neste trabalho, apenas em triangulações. A Figura 2.1 nos mostra uma possível triangulação de um objeto bidimensional.

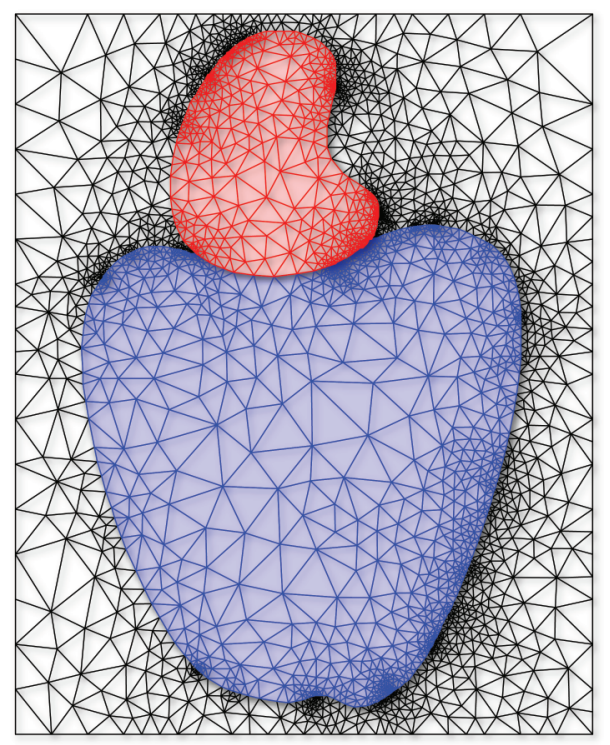

Figura 2.1: Malha de triângulos ou triangulação (veja (Lizier (2010))).

Técnicas de geração de malhas são amplamente empregadas em vários campos da engenharia, computação gráfica, entre outros, e fazem uso de modelos físicos e matemáticos. A simulação do fluxo sanguíneo e simulação do comportamento mecânico do pulmão são exemplos de problemas que precisam de malhas adequadas para solução numérica de fenômenos físicos.

Um dos métodos mais utilizados na geração de malhas é o de elementos finitos (Frey \& George (2000)), no qual um problema contínuo é substituído por um problema discreto que pode realmente ser computado. A solução para este problema discreto é, então, uma solução aproximada para o problema original, cuja precisão é baseada em diversas opções feitas no processo numérico.

A geração de malhas de elementos finitos consiste, de uma forma geral, na divisão de um determinado domínio de interesse em sub-domínios. Domínios bidimensionais podem ser divididos em quadriláteros ou triângulos, enquanto que domínios tridimensionais podem ser divididos em tetraedros, pentaedros ou hexaedros. 
Na geração de malhas de superfícies em três dimensões, problemas como a garantia de topologia e continuidade para os vértices de uma malha poligonal de topologia arbitrária tem sido de grande interesse de pesquisa ao longo dos anos.

Antes de definir a modelagem do problema de otimização, precisamos ainda descrever o que consideramos uma malha fina (densa) e uma malha grossa (simplificada). Informalmente, dada uma triangulação de uma superfície, a qual chamamos de malha fina (veja a Figura 2.2 ), escolhemos alguns pontos desta triangulação para gerar uma nova malha da mesma superfície, porém, menos densa, a qual chamamos de malha grossa (veja a Figura 2.3). Como podemos observar nas Figuras 2.2 e 2.3, a malha grossa contém menos detalhes sobre a superfície do que a malha fina. No entanto, a malha grossa necessita que uma quantidade muito menor de dados seja armazenada.

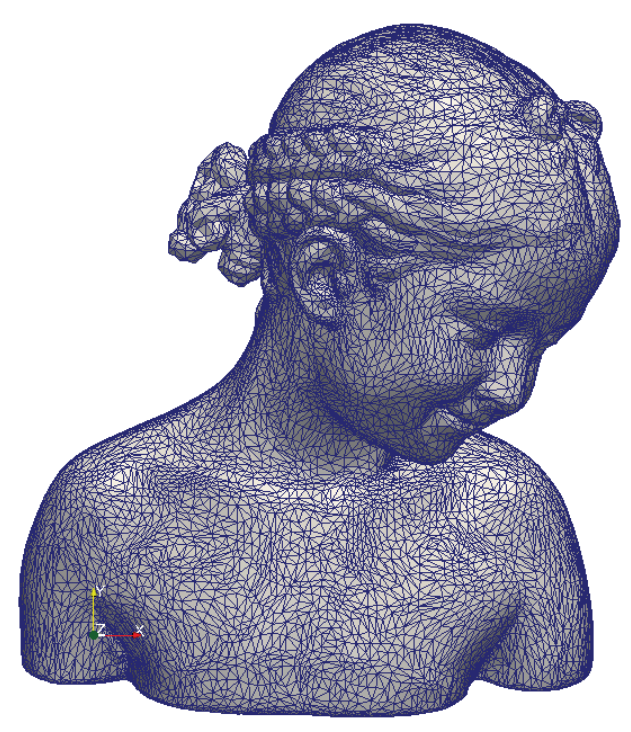

Figura 2.2: Exemplo de malha fina.

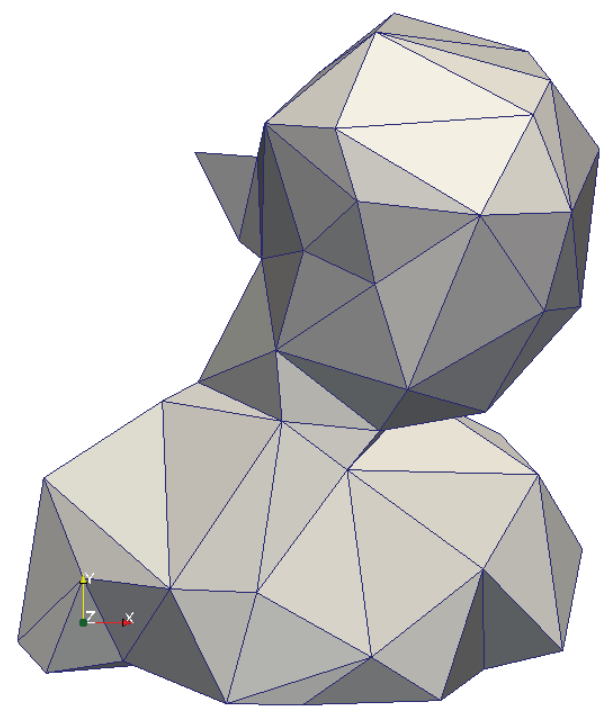

Figura 2.3: Exemplo de malha grossa.

Daqui em diante usaremos o conceito de estrela de um vértice. Vejamos a seguir sua definição formal.

Definição 2.3. Seja $\mathcal{K}$ um complexo simplicial em $\mathbb{E}^{n}$. Então, para qualquer simplexo $\sigma$ em $\mathcal{K}$, definimos os conjuntos

$$
s t(\sigma, \mathcal{K})=\{\tau \in \mathcal{K} \mid \exists \eta \text { em } \mathcal{K} \text { tal que } \sigma \text { é uma face de } \eta \text { e } \tau \text { é uma face de } \eta\}
$$

$e$

$$
l k(\sigma, \mathcal{K})=\{\tau \in \mathcal{K} \mid \tau \text { está em } s t(\sigma, \mathcal{K}) \text { e } \tau \text { e } \sigma \text { não tem faces em comum }\}
$$

chamados estrela e vizinhança de $\sigma$ em $\mathcal{K}$, respectivamente. Note que o conjunto $s t(\sigma, \mathcal{K})$ é composto por $\sigma$, todos os simplexos de $\mathcal{K}$ que tem $\sigma$ como uma face, e todos os simplexos de $\mathcal{K}$ 
que são faces de algum simplexo que tem $\sigma$ como uma face. Por sua vez, o conjunto $l k(\sigma, K)$ é composto de todos os simplexos em st $(\sigma, K)$ que não contêm $\sigma$ como uma face. Além disso, ambos conjuntos $l k(\sigma, K)$ e st $(\sigma, K)$ são complexos simpliciais e st $(\sigma, K)$ é sempre não vazio.

A Figura 2.4 ilustra os conjuntos $\operatorname{st}(\sigma, K)$ e $1 \mathrm{k}(\sigma, K)$ da Definição 2.3.

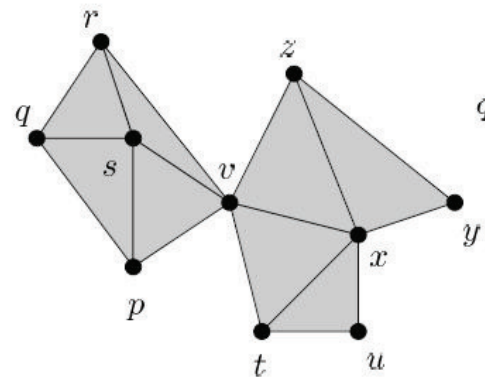

(a)

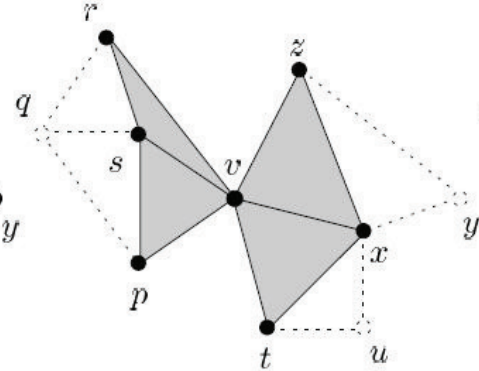

(b)

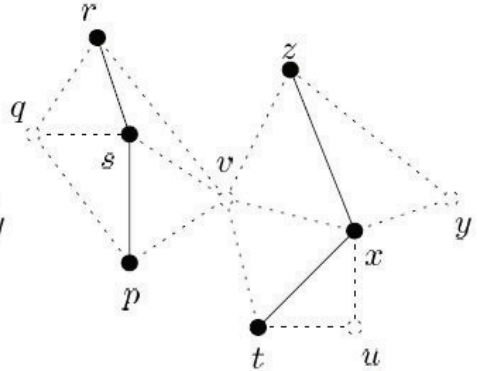

(c)

Figura 2.4: Um complexo simplicial $K$ (a), uma estrela do vértice $v$, st $(\sigma, K)$ (b) e uma vizinhança do vértice $v, \operatorname{lk}(\sigma, K)$ (c).

Informalmente, a estrela de um vértice $v$ é definida como o conjunto de vértices que possuem alguma aresta em comum com $v$. A Figura 2.5 é uma ilustração de uma estrela de um vértice $v$ (central) de uma malha.

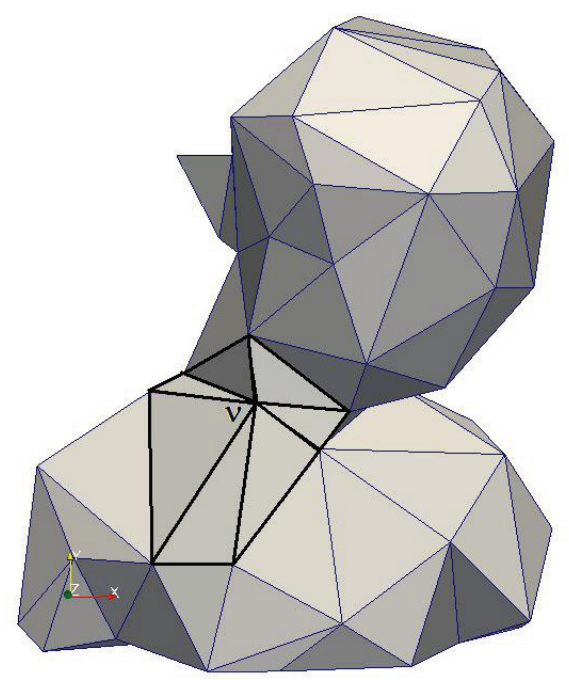

Figura 2.5: Exemplo de estrela de um vértice $v$ da malha grossa apresentada na Figura 2.3.

\subsection{Otimização de malhas}

Agora que já definimos o que é uma malha, queremos alguma maneira de medir sua qualidade e melhorá-la. Optamos por melhorar malhas grossas, já que para muitas aplicações, a 
malha grossa se comporta de maneira eficiente, contendo informações da malha fina e necessitando de muito menos vértices, arestas e faces em sua representação.

As técnicas voltadas para a modelagem de imagem buscam gerar malhas que minimizem um erro de aproximação entre a imagem original e a imagem representada pela malha. Estas técnicas podem ser descritas basicamente por duas etapas: pré-processamento e geração da malha. Muitas vezes, a malha gerada é de má qualidade, fazendo com que essas técnicas proponham uma etapa de pós-processamento, visando a melhoria dos elementos desta malha. A suavização Laplaciana (Field (1988)) e o uso de molas (Lohner (1987)) são abordagens muito utilizadas na etapa de pós-processamento (melhoria da malha). Estudamos aqui um meio de utilizar um método clássico de otimização não-linear para melhoria dos elementos (triângulos) parametrizados de uma malha grossa, incluindo-o na etapa de pós-processamento.

Para este fim, necessitamos descrever o problema de programação não-linear a ser abordado na etapa de melhoria de malhas, a qual chamamos de otimização de malhas de superfícies. Mais especificamente, consideramos malhas de superfícies em três dimensões e o processo de otimização é descrito para retalhos parametrizados destas malhas.

Parametrizações de superfícies de malhas de triângulos são usadas em muitas aplicações de processamento de malhas como, por exemplo, simulações gráficas. Muitas vezes, é necessário preservar a métrica da superfície e, assim, minimizar a deformação do ângulo e da área dos triângulos parametrizados da malha. Muitas técnicas privilegiam apenas a preservação do ângulo ou a preservação da área. Em (Degener (2003)) é proposta uma fórmula de energia que mede simultaneamente as deformações de ângulo e área global imposta por uma parametrização. Estamos interessados neste tipo de abordagem.

Todo o processo de otimização que vamos apresentar ocorrerá nas coordenadas bidimensionais das estrelas da malha fina contidas em cada uma das estrelas de cada vértice da malha grossa. Para cada vértice $v$ da malha grossa, determinamos sua estrela e o conjuto $V$ de vértices da malha fina que pertencem a esta estrela. Este conjunto $V$ de vértices será parametrizado e, para cada vértice $v p$ da parametrização, utilizamos algum solver de programação não-linear para resolver um problema otimização não-linear que determina uma nova posição para o vértice $v p$ de forma que a distorção da malha obtida seja diminuida. Após todos os vértices da parametrização serem otimizados (movidos), atualizamos o vértice da malha grossa, na superfíce, a partir do seu vértice correspondente na malha fina. Note que apenas a malha grossa será modificada, mas isso será feito com base na melhora da distorção da parametrização dos vértices da malha fina.

Na Seção 2.2.1 descrevemos um modelo matemático para o problema de otimização a ser resolvido para cada vértice da parametrização, o qual podemos resolver utilizando algum solver de programação não-linear. Para mais informações sobre os conceitos utilizados na Seção 2.2.1, consulte o Apêndice B. 


\subsubsection{Modelo de otimização não-linear}

Para cada vértice $v p$ parametrizado da malha fina, consideramos sua estrela e definimos um problema de otimização não-linear que, quando resolvido, gera uma nova posição para o vértice $v p$. Após a movimentação de todos os vértices da malha fina, os vértices da malha grossa serão movimentados de maneira correspondente. Nesta seção, descrevemos o problema de programação não-linear a ser resolvido para cada vértice da malha fina parametrizada.

Dada uma superfície $S \subset \mathbb{R}^{k}$, uma parametrização é definida como um homeomorfismo

$$
\begin{aligned}
\phi: \Omega \subset \mathbb{R}^{2} & \rightarrow S \\
(u, v) & \mapsto \phi(u, v),
\end{aligned}
$$

a partir do espaço de parâmetros $\Omega$ em $S$. Em seguida, considera-se o problema de encontrar uma parametrização de um conjunto $S$ que tem uma triangulação

$$
\mathcal{M}^{\prime}=\left\{[1 \ldots n], \mathcal{T},\left(p_{i}\right)_{i=1 \ldots n}\right\}
$$

onde $[1 \ldots n]$ indica os vértices, $\mathcal{T} \subset[1 \ldots n]^{3}$ representa os triângulos e $p_{i}$ é a localização do vértice $i$ em $S$. Além disso, exige-se uma parametrização inversa $\psi:=\phi^{-1}$ linear nos triângulos de $\mathcal{M}^{\prime}$. Esse mapeamento $\psi$ é unicamente determinado pelos seus valores $\left(\left(u_{i}, v_{i}\right)\right)_{i=1 \ldots n}:=$ $\left(\psi\left(p_{i}\right)\right)_{i=1 \ldots n}$ nos vértices da malha e

$$
\mathcal{M}=\left\{[1 \ldots n], \mathcal{T},\left(\left(u_{i}, v_{i}\right)\right)_{i=1 \ldots n}\right\}
$$

é um parâmetro da triangulação do domínio para a imagem $\psi(S)$. A parametrização inversa $\psi$ mapeia vértices e faces de $\mathcal{M}^{\prime}$ em vértices e faces de $\mathcal{M}$, respectivamente.

Dada uma parametrização diferenciável

$$
\phi: \Omega \subset \mathbb{R}^{2} \rightarrow S \subset \mathbb{R}^{k}
$$

a primeira forma fundamental $\mathbf{I}_{\phi}$, que capta a estrutura métrica de $S$, é definida como

$$
\mathbf{I}_{\phi}=\nabla^{t} \phi \cdot \nabla \phi=\left(\begin{array}{ll}
a & b \\
b & c
\end{array}\right)
$$

com $a=\left\|\frac{\partial \phi}{\partial u}\right\|^{2}, b=\left\langle\frac{\partial \phi}{\partial u}, \frac{\partial \phi}{\partial v}\right\rangle$ e $c=\left\|\frac{\partial \phi}{\partial v}\right\|^{2}$. A matriz $\mathbf{I}_{\phi} \in \mathbb{R}^{2 \times 2}$ é simétrica, definida positiva $\mathrm{e}$, em cada $\omega \in \Omega$, induz um produto escalar em $\mathbb{R}^{2}$, que descreve os comprimentos e ângulos dos vetores em $\mathbb{R}^{2}$ depois de ser mapeado por $\mathbf{I}_{\phi}$.

A parametrização é dita conformal se, para todo $\omega \in \Omega$,

$$
\mathbf{I}_{\phi}(\omega)=\lambda(\omega) \cdot \mathbf{I},
$$


onde I denota a matriz identidade em $\mathbb{R}^{2 \times 2}$.

Em outras palavras, as derivadas das $u$-curvas e $v$-curvas passando por $\phi(\omega)$ são ortogonais e tem a mesma magnitude, que é consistente com o fato de que $\phi$ é uma parametrização conformal. Isso é equivalente a dizer que os dois autovalores da matriz associada de $\mathbf{I}_{\phi(\omega)}$ são ambos iguais para $\lambda(\omega)$. Em geral, se $\phi$ é uma parametrização de $S$, e se $\lambda_{\max }$ e $\lambda_{\min }$ são os autovalores máximo e mínimo de $\mathbf{I}_{\phi(\omega)}$, então a conformidade de $X$ pode ser expressada por

$$
\frac{\lambda_{\max }}{\lambda_{\min }}
$$

Como $\mathbf{I}_{\phi}(\omega)$ é definida positiva, temos $\lambda_{\max } \geq \lambda_{\min }>0$. Então, o menor valor que a razão $\frac{\lambda_{\max }}{\lambda_{\min }}$ pode assumir é 1. Dai, $\frac{\lambda_{\max }}{\lambda_{\min }}=1$.

A condição de conformalidade permite que as derivadas direcionais sejam uniformemente escaladas por um fator $\lambda(\omega)$ (autovalor do mapa $\mathbf{I}_{\phi}(\omega)$ ), que pode variar deslocando-se de um ponto a outro na superfície. Se este fator não é igual a 1, uma forma no domínio aparece esticada ou encolhida quando é mapeada para a superfície e sua área é distorcida.

Como $\lambda(\omega)$ é contínuo, em torno de cada $\omega \in \Omega$ existe uma vizinhança suficientemente pequena na qual a variação de $\lambda(\omega)$ é arbitrariamente pequena. Assim, a área é localmente, mas não globalmente, preservada por um mapa conformal. Mapas conformais são, portanto, adequados para aplicações onde a preservação do ângulo é obrigatória, mas a preservação da área global é menos importante. Se, além de ângulos, a área deve ser preservada globalmente, a magnitude das derivadas direcionais deve ser fixada, levando à noção de isometria.

A parametrização é dita isométrica se

$$
\lambda(\omega)=1
$$

para todos $\omega \in \Omega$. Ou seja, a primeira forma fundamental é igual à matriz identidade em cada ponto.

Para encontrar a área de deformação imposta por um mapa $\phi$, consideramos um eixo suficientemente pequeno com quadrados alinhados em $\Omega$ com área $A$. A imagem deste quadrado é um trapézio estendido entre as derivadas direcionais em $u$ e $v$, cuja área é dada por $A \cdot \sqrt{\operatorname{det} \mathbf{I}_{\phi}}$. Assim, $\phi$ preserva a área se, e somente se,

$$
\sqrt{\operatorname{det} \mathbf{I}_{\phi}}=1
$$

Gostariamos, então, de encontrar uma parametrização $\phi$ que satisfaça (2.4) e (2.5). Para isso, movemos os vértices da malha fina (o que irá gerar um movimento dos vértices na malha grossa) de forma que a parametrização usada, proposta em (Siqueira (2009)), gere uma distorção menor dos vértices da malha grossa em relação aos vértices da superfície. 
Para impor a condição de preservação de área, (Degener (2003)) propõe $f(x)=x+\frac{1}{x}$ como função objetivo, uma vez que é convexa, para $x>0$, e alcança o seu valor mínimo em 1 . Além disso, ela cresce para o infinito quando $x \rightarrow 0$ ou $x \rightarrow \infty$. Usamos $f(x)$ para definir a função objetivo que é uma combinação das energias de deformação de área e de ângulo. Para o caso da energia de deformação da área, temos

$$
E_{\text {area }}(\omega)=f\left(\sqrt{\operatorname{det} \mathbf{I}_{\phi}}(\omega)\right)=\sqrt{\operatorname{det} \mathbf{I}_{\phi}}(\omega)+\frac{1}{\sqrt{\operatorname{det} \mathbf{I}_{\phi}}(\omega)}
$$

obtida substituindo $x$ por $\sqrt{\operatorname{det} \mathbf{I}_{\phi}}(\omega)$. Esta propriedade garante que a orientação de todas as faces é preservada durante a minimização e, assim, não é possível inverter faces. Usando a mesma função para a deformação angular, temos a energia conformal

$$
E_{\text {angulo }}(\omega)=f\left(\sqrt{\frac{\lambda_{\max }}{\lambda_{\min }}}\right)=\sqrt{\frac{\lambda_{\max }}{\lambda_{\min }}}+\sqrt{\frac{\lambda_{\min }}{\lambda_{\max }}} .
$$

Sabemos que a minimização da distorção da área só é possível em teoria, já que, na prática, causa muitos problemas numéricos. Assim, a energia combinada introduzida em (Degener (2003)) é dada por

$$
E_{\text {combinada }}(\omega)=E_{\text {angulo }}(\omega) \cdot\left(E_{\text {area }}(\omega)\right)^{\theta}
$$

onde o parâmetro $\theta$ varia entre 0 e $\infty$ e controla a importância relativa da preservação da área e ângulo.

Atribuindo $\theta=1$, temos

$$
\begin{aligned}
E_{\text {angulo }}(\omega) \cdot E_{\text {area }}(\omega) & =f\left(\sqrt{\frac{\lambda_{\max }}{\lambda_{\min }}}\right) \cdot f\left(\sqrt{\operatorname{det} \mathbf{I}_{\phi}}(\omega)\right) \\
& =f\left(\lambda_{\max }\right)+f\left(\lambda_{\min }\right) .
\end{aligned}
$$

Podemos definir uma parametrização $\phi$ como uma combinação de distorção de área e ângulo, se integrarmos os retalhos sobre a superfície $S$

$$
E(\phi)=\int_{S} E_{\text {combinada }}\left(\phi^{-1}(p)\right) d p
$$

Para o caso especial de uma parametrização linear por partes sobre uma triangulação, $\nabla \phi$ e $\mathbf{I}_{\phi}$ são constantes dentro de cada triângulo de $\mathcal{M}$ que, por sua vez, faz com que as energias $E_{\text {area }}(\omega), E_{\text {angulo }}(\omega)$ e $E_{\text {combinada }}(\omega)$ sejam constantes dentro de cada triângulo.

A energia linear do mapa $\left.\phi\right|_{\triangle_{\mathcal{M}}(T)}$, ou seja, a energia angular, pode ser escrita como

$$
E_{\text {angulo }}(T)=\frac{\cot \alpha|a|^{2}+\cot \beta|b|^{2}+\cot \gamma|c|^{2}}{2 \operatorname{area}\left(\triangle_{\mathcal{M}}(T)\right)},
$$




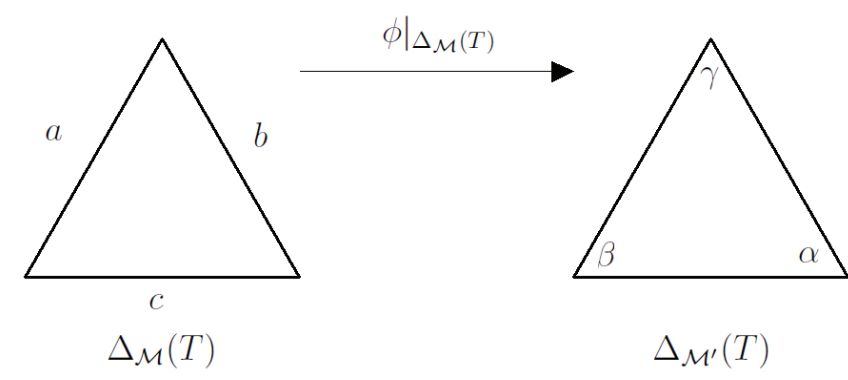

Figura 2.6: A restrição de $\phi$ a um triângulo $\triangle_{\mathcal{M}^{\prime}}(T)$.

onde

$$
\begin{aligned}
& a=\sqrt{\left(x_{2}-x_{1}\right)^{2}+\left(y_{2}-y_{1}\right)^{2}}, \\
& b=\sqrt{\left(x_{2}-x\right)^{2}+\left(y_{2}-y\right)^{2}} \\
& c=\sqrt{\left(x-x_{1}\right)^{2}+\left(y-y_{1}\right)^{2}}
\end{aligned}
$$

$\alpha$ é o ângulo oposto ao lado $a, \beta$ é o ângulo oposto ao lado $b, \gamma$ é o ângulo oposto ao lado $c \mathrm{e}$ area $\left(\triangle_{\mathcal{M}}(T)\right)$ é a área do triângulo $T$ sem ser parametrizado (veja a figura 2.7).

A energia da área pode ser escrita como

$$
E_{\text {area }}(T)=\frac{\operatorname{area}\left(\triangle_{\mathcal{M}^{\prime}}(T)\right)}{\operatorname{area}\left(\triangle_{\mathcal{M}}(T)\right)}+\frac{\operatorname{area}\left(\triangle_{\mathcal{M}}(T)\right)}{\operatorname{area}\left(\triangle_{\mathcal{M}^{\prime}}(T)\right)}
$$

onde area $\left(\triangle_{\mathcal{M}^{\prime}}(T)\right)$ é a área do triângulo $T$ da parametrização $\mathcal{M}^{\prime}$ e area $\left(\triangle_{\mathcal{M}}(T)\right)$ é a área do triângulo $T$ sem ser parametrizado.

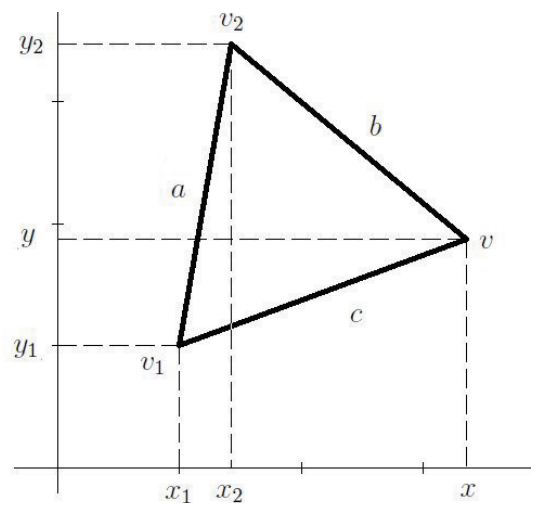

Figura 2.7: Um triângulo de uma estrela.

Até agora consideramos triângulos da malha. A partir deste momento, consideramos a estrela de cada vértice $v$, que é o vértice que será movido. Calculamos então a energia $E(\phi)$ de 
um mapa $\phi$ considerando todos os triângulos de uma estrela que tem como variável seu vértice central.

Finalmente, a integral torna-se a soma finita

$$
E(\phi)=\sum_{T \in \mathcal{T}} E_{T} \cdot \operatorname{area}\left(\triangle_{\mathcal{M}^{\prime}}(T)\right)
$$

onde

$$
E_{T}=E_{\text {angulo }}(T) \cdot\left(E_{\text {area }}(T)\right)^{\theta}
$$

só depende das coordenadas da parametrização e das coordenadas da superfície dos três vértices em $T$.

Perceba que as únicas variáveis do problema são as coordenadas $(x, y)$ do vértice central da estrela que será movido, pois $E_{\text {angulo }}(T)$ tem como variáveis $(x, y)$ e podemos ainda deduzir as áreas area $\left(\triangle_{\mathcal{M}}(T)\right)$ e area $\left(\triangle_{\mathcal{M}^{\prime}}(T)\right)$ em função de $a, b$ e $c$, ou seja, $E_{\text {area }}(T)$ também possui como variáveis $(x, y)$.

Logo, as variáveis da energia $E(\phi)$ são $x$ e $y$ a denotaremos por $E_{\phi}(x, y)$, já que é a energia relacionada ao mapa $\phi$, quando variamos $(x, y)$. Agora que encontramos a energia $E_{\phi}(x, y)$ que vamos minimizar, precisamos encontrar as restrições do problema.

Considerando uma estrela da malha fina, queremos que a nova posição do vértice esteja dentro da estrela (veja a Figura 2.8). Considerando $v=(x, y)$ como a variável do problema, o mesmo não pode sair da região definida pelo polígono $\left(v_{1}, v_{2}, \ldots, v_{n}\right)$, onde $n$ é o grau da estrela. Assim, precisamos escrever as retas que passam por $v_{1} v_{2}, v_{2} v_{3}, \ldots, v_{n} v_{1}$.

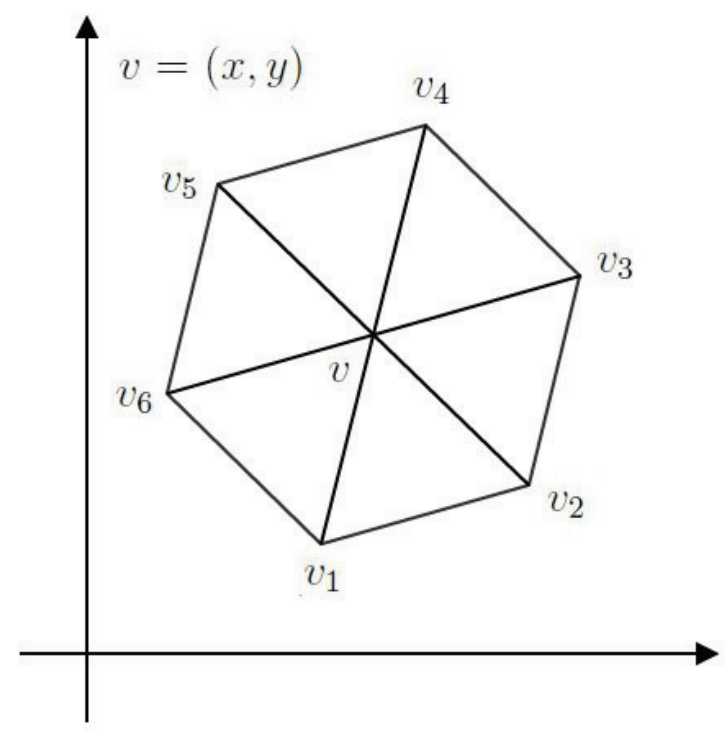

Figura 2.8: Restrições impostas a uma estrela. 
Para o exemplo da Figura 2.8, temos:

$$
\begin{aligned}
& \left(\left(y_{2}-y_{1}\right)^{2} \cdot x\right)+\left(\left(x_{1}-x_{2}\right)^{2} \cdot y\right)+\left(x_{2} \cdot y_{1}\right)-\left(y_{2} \cdot x_{1}\right) \leq 0, \\
& \left(\left(y_{3}-y_{2}\right)^{2} \cdot x\right)+\left(\left(x_{2}-x_{3}\right)^{2} \cdot y\right)+\left(x_{3} \cdot y_{2}\right)-\left(y_{3} \cdot x_{2}\right) \leq 0, \\
& \left(\left(y_{4}-y_{3}\right)^{2} \cdot x\right)+\left(\left(x_{3}-x_{4}\right)^{2} \cdot y\right)+\left(x_{4} \cdot y_{3}\right)-\left(y_{4} \cdot x_{3}\right) \leq 0, \\
& \left(\left(y_{5}-y_{4}\right)^{2} \cdot x\right)+\left(\left(x_{4}-x_{5}\right)^{2} \cdot y\right)+\left(x_{5} \cdot y_{4}\right)-\left(y_{5} \cdot x_{4}\right) \leq 0, \\
& \left(\left(y_{6}-y_{5}\right)^{2} \cdot x\right)+\left(\left(x_{5}-x_{6}\right)^{2} \cdot y\right)+\left(x_{6} \cdot y_{5}\right)-\left(y_{6} \cdot x_{5}\right) \leq 0, \\
& \left(\left(y_{1}-y_{6}\right)^{2} \cdot x\right)+\left(\left(x_{6}-x_{1}\right)^{2} \cdot y\right)+\left(x_{1} \cdot y_{6}\right)-\left(y_{1} \cdot x_{6}\right) \leq 0 .
\end{aligned}
$$

Generalizando, como temos $v_{i}=\left(x_{i}, y_{i}\right)$ conhecidos, podemos escrever todas as restrições em função dp vértice central da estrela $v=(x, y)$ como sendo

$$
\begin{gathered}
\left(\left(y_{i+1}-y_{i}\right)^{2} \cdot x\right)+\left(\left(x_{i}-x_{i+1}\right)^{2} \cdot y\right) \\
\quad+\left(x_{i+1} \cdot y_{i}\right)-\left(y_{i+1} \cdot x_{i}\right) \leq 0
\end{gathered}
$$

onde $i=1, \ldots, n, n$ é o grau da uma estrela e o índice $n+1$ corresponde ao índice 1 .

Para simplificar essa restrição definimos

$$
\begin{aligned}
& \left(y_{i+1}-y_{i}\right)=\Lambda_{i}, \\
& \left(x_{i}-x_{i+1}\right)=\Gamma_{i} \\
& \left(\left(x_{i+1} \cdot y_{i}\right)-\left(y_{i+} \cdot x_{i}\right)\right)=\Upsilon_{i} .
\end{aligned}
$$

Assim, as restrições podem ser escritas como

$$
\Lambda_{i}^{2} \cdot x+\Gamma_{i}^{2} \cdot y+\Upsilon_{i} \leq 0
$$

Desta maneira, para cada estrela de uma malha fina, temos que resolver um problema do tipo

$$
\begin{array}{ll}
\text { Minimizar } & F(x, y)=E(\phi)=\sum_{T \in \mathcal{T}} E_{T} \cdot \operatorname{area}\left(\triangle_{\mathcal{M}^{\prime}}(T)\right) \\
\text { sujeito a } & \Lambda_{i}^{2} \cdot x+\Gamma_{i}^{2} \cdot y+\Upsilon_{i} \leq 0, \quad i=1, \ldots, n
\end{array}
$$

onde:

- $x$ e $y$ são as variáveis do problema;

- $\mathcal{T}$ é o conjunto de todos os triângulos da estrela dada;

- $E_{T}=E_{\text {angulo }}(T) \cdot\left(E_{\text {area }}(T)\right)^{\theta}$, onde $E_{\text {angulo }}(T)$ pode ser encontrada em (2.7) e $E_{\text {area }}(T)$ pode ser encontrada em (2.9);

- $\operatorname{area}\left(\triangle_{\mathcal{M}^{\prime}}(T)\right)$ é a área de cada triângulo da estrela na parametrização; 
- $\Lambda_{i}, \Gamma_{i}$ e $\Upsilon_{i}$ são definidos em (2.10);

- $n$ é o grau de uma estrela.

No próximo capítulo, apresentamos detalhes de implementação da estrutura de dados utilizada para auxiliar na resolução do problema de otimização de malhas e da implementação da rotina de otimização. 


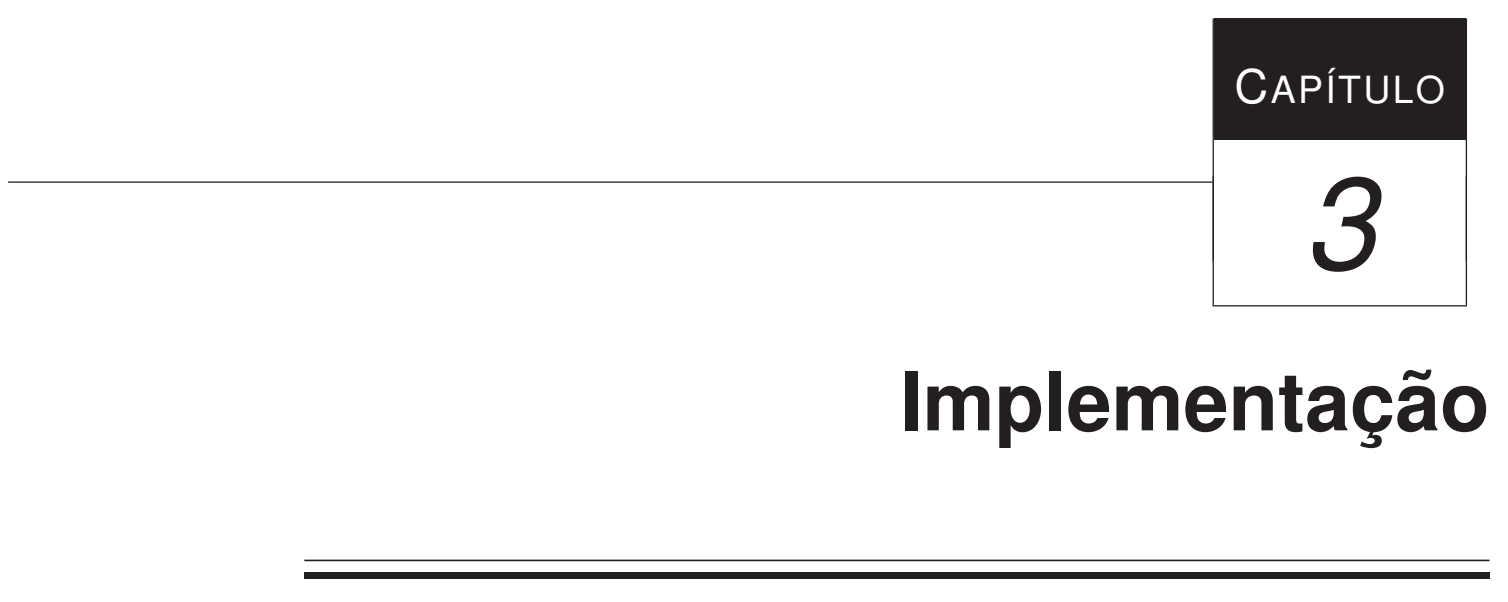

Neste capítulo apresentamos detalhes da implementação do método de otimização descrito no Capítulo 2.

\subsection{Estrutura de dados}

Para armazenar uma malha computacionalmente, precisamos ter em mente quais informações serão necessárias, como, por exemplo: dado um vértice, a quais faces ele pertence? Dada uma face, quais vértices pertencem a ela? A estrutura de dados deve armazenar essas informações, e muitas mais que vamos descrever, de maneira que não haja ambiguidades, que o acesso e a alteração destas informações sejam o mais eficiente possível.

Para a execução da otimização em uma malha, precisamos de uma estrutura de dados adequada que armazene uma malha de triângulos grossa e uma malha de triângulos fina. A estrutura de dados que será descrita deve conter informações de faces, arestas, vértices, entre outras. Usamos a estrutura de dados baseada em halfedge (McGuire (2000)), que está apta a representar malhas que não possuem pontos ou vértices complexos, nem superfícies que possuam aberturas.

Esta estrutura de dados transforma cada aresta da malha em um par de "meias arestas"(halfedge, em inglês). Uma halfedge do par tem orientação oposta à outra halfedge. A Figura 3.1 mostra uma malha triangular formada por quatro vértices. Cada seta é uma halfedge que liga um vértice a outro. Nesta estrutura, as halfedges são orientadas consistentemente, no sentido anti-horário, ao redor da face e ao longo de cada fronteira. 


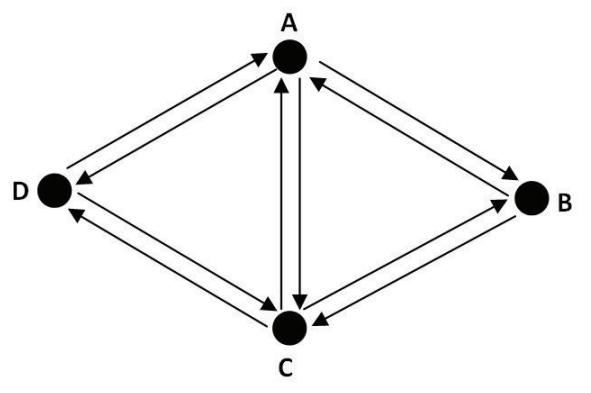

Figura 3.1: Representação da estrutura de dados halfedge.

Para cada halfedge, é possível encontrar seu vértice inicial, qual é a face adjacente, a halfedge anterior, a halfedge posterior e a halfedge oposta. Também necessitamos que cada vértice faça referência a uma das halfedges que o tem como vértice inicial, que cada face faça referência a uma das halfedges que a forma, e que cada aresta faça referência a uma de suas halfedges. Com essa estrutura, podemos percorrer todos os vértices, arestas e faces de uma malha. A Figura 3.2, ilustra a estrutura de halfedges.

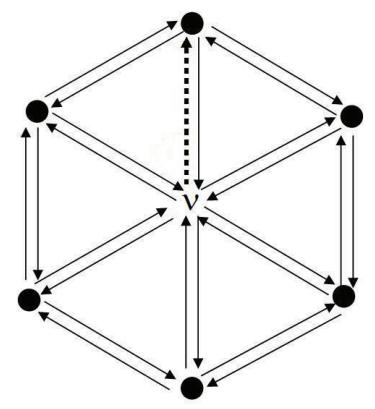

Halfedge (pontilhada) aponta para seu vértice inicial $\nu$.

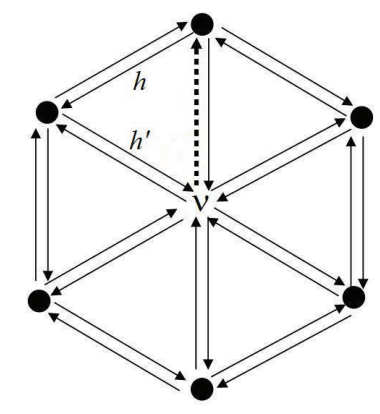

Halfedge (pontilhada) aponta para a halfedge anterior e posterior, $h$ ' $e$, respectivamente.

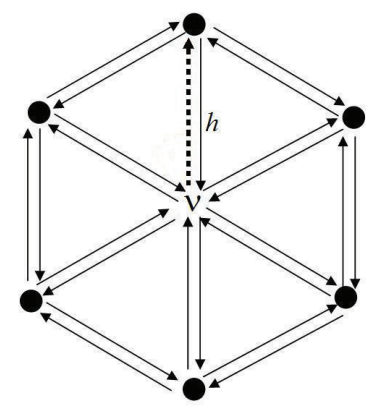

Halfedge (pontilhada) aponta para sua oposta, $h$.

Figura 3.2: Ilustração da estrutura de dados halfedge.

Implementamos, em $\mathrm{C}++$, uma estrutura de dados baseada em halfedges para representar as malhas grossa e fina. Esta implementação foi baseada na implementação de (Siqueira (2009)). A seguir, descrevemos alguns detalhes desta estrutura de dados.

\subsubsection{Estrutura de dados da malha grossa}

As principais classes utilizadas para representar a malha grossa são as seguintes:

\section{- sVertex}

Esta classe é usada para representar um vértice $s v$ da malha grossa. Ela possui:

- uma variável $i d$, do tipo inteiro, que armazena o número de identificação do vértice $s v$ da malha grossa; 
- variáveis $x, y$ e $z$, do tipo real, que armazenam as coordenadas do vértice $s v$ em $\mathbb{R}^{3}$;

- um ponteiro para um objeto da classe sHalfedge (veja a seguir), que aponta para uma das halfedges, da malha grossa, que tem o vértice $s v$ como vértice inicial.

\section{- sFace}

Esta classe é usada para representar uma face $s f$ da malha grossa. Ela possui:

- uma variável $i d$, do tipo inteiro, que armazena um número de identificação da face sf da malha grossa;

- um ponteiro para um objeto da classe sHalfedge (veja a seguir), que aponta para uma das halfedges, da malha grossa, que formam a face $s f$;

- uma lista que contém todos os vértices da malha fina que pertencem à face $s f$ da malha grossa.

\section{- sHalfedge}

Esta classe é usada para representar uma halfedge sh da malha grossa. Ela possui:

- um ponteiro para um objeto da classe sVertex, que aponta para o vértice inicial da halfedge sh;

- um ponteiro para um objeto da classe sHalfedge, que aponta para a halfedge posterior a sh, segundo a orientação na face;

- um ponteiro para um objeto da classe sHalfedge, que aponta para a halfedge anterior a sh, segundo a orientação na face.

\subsubsection{Estrutura de dados da malha fina}

As principais classes utilizadas para representar a malha fina são as seguintes:

\section{- fVertex}

Esta classe é usada para representar um vértice $f v$ da malha fina. Ela possui:

- uma variável $i d$, do tipo inteiro, que armazena o número de identificação do vértice $f v$ da malha fina;

- variáveis $x, y$ e $z$, do tipo real, que armazenam as coordenadas do vértice $f v$ em $\mathbb{R}^{3}$;

- variáveis $u$ e $w$, do tipo real, que armazenam as coordenadas baricêntricas do vértice $f v$, em $\mathbb{R}^{2}$, em relação ao triângulo da malha grossa ao qual $f v$ pertence;

- um ponteiro para um objeto da classe fHalfedge (veja a seguir), que aponta para uma das halfedges da malha fina, que tem o vértice $f v$ como vértice inicial; 
- um ponteiro para um objeto da classe sHalfedge, que aponta para uma das halfedges que formam uma face da malha grossa, à qual o vértice $f v$ da malha fina pertence;

- um ponteiro para um objeto da classe sFace, que aponta para a face $s f$ da malha grossa à qual o vértice $f v$ pertence.

\section{- fFace}

Esta classe é usada para representar uma face $f f$ da malha fina. Ela possui:

- uma variável $i d$, do tipo inteiro, que armazena um número de identificação da face ff da malha fina;

- um ponteiro para um objeto da classe fHalfedge (veja a seguir), que aponta para uma das halfedges, da malha fina, que formam a face $f f$.

\section{- fHalfedge}

Esta classe é usada para representar uma halfedge fh da malha fina. Ela possui:

- um ponteiro para um objeto da classe fVertex, que aponta para o vértice inicial da halfedge;

- um ponteiro para um objeto da classe fHalfedge, que aponta para a halfedge posterior a fh, segundo a orientação na face;

- um ponteiro para um objeto da classe fHalfedge, que aponta para a halfedge anterior a fh, segundo a orientação na face.

A Figura 3.3 ilustra a estrutura de dados da malha fina e da malha grossa baseada na estrutura de halfedges.

\subsection{Arquivos de entrada e saída}

A leitura de dados das malhas fina e grossa é feita através de arquivos de entrada. Necessitamos de quatro arquivos iniciais, descrito a seguir.

\section{- f-nome.off}

Este é um arquivo de dados, no formato $\mathrm{OFF}^{1}$, que fornece ao programa todos os vértices da malha grossa e ligações (arestas) existente entre eles.

\section{- s-nome.off}

Este arquivo, também no formato OFF, fornece ao programa todos os vértices da malha fina e as ligações (arestas) entre eles.

\footnotetext{
${ }^{1}$ OFF, Object File Format, encontrado em http://www.geomview.org.html
} 


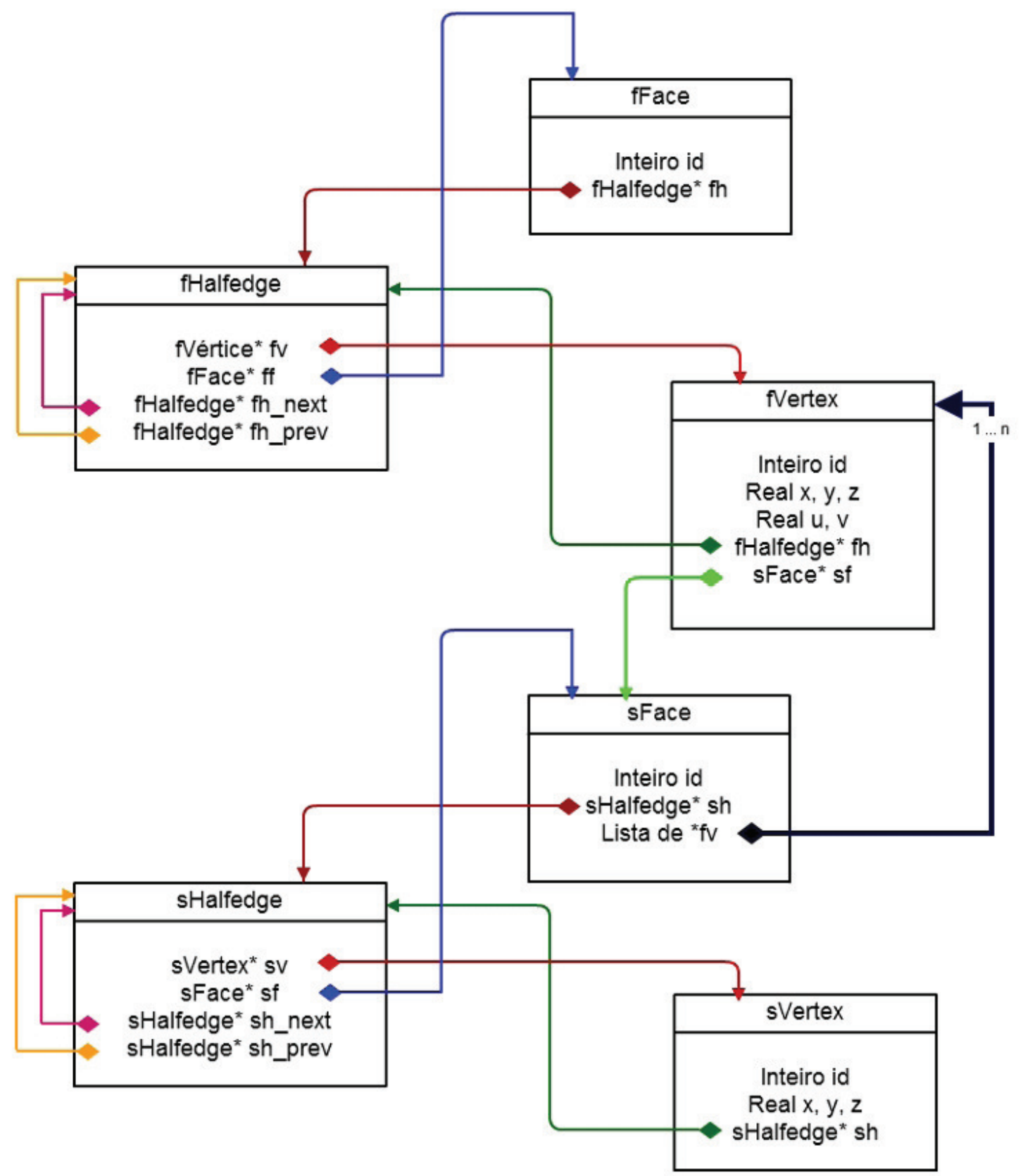

Figura 3.3: Ilustração das estruturas de dados das malhas grossa e fina, baseadas na estrutura de halfedges.

- soff-nome.soff

Este arquivo contém informações sobre a malha grossa e a malha fina. Fornece ao programa uma listagem de todos os vértices da malha grossa e a lista de vértices da malha fina pertencentes à estrela de cada vértice da malha, juntamente com suas coordenadas baricêntricas.

O esquema abaixo apresenta o formato de um arquivo SOFF. 


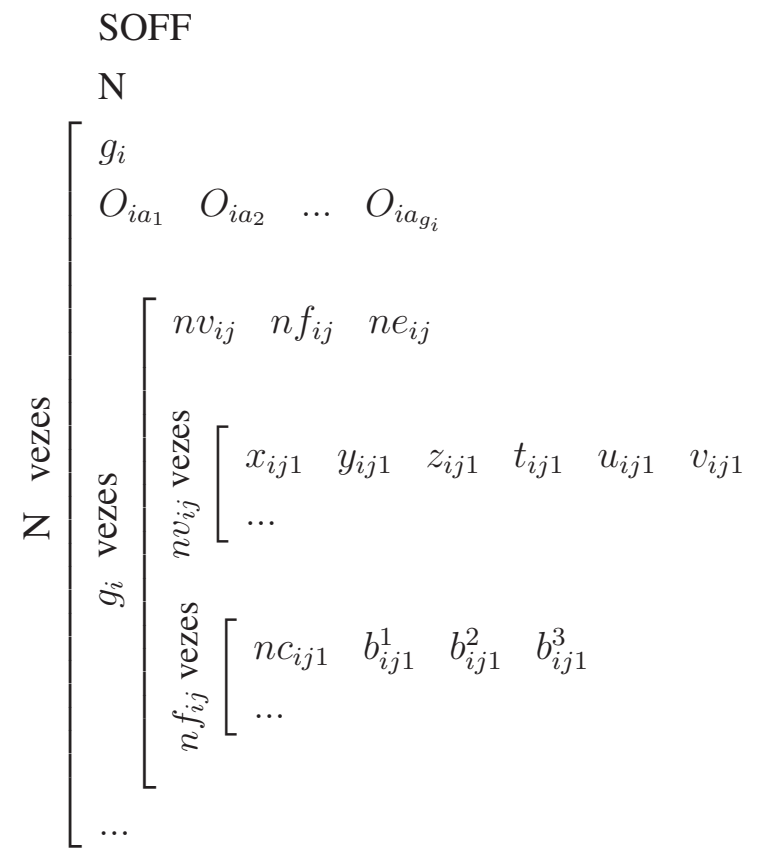

Descrevemos aqui seus detalhes:

- SOFF indica a extensão do arquivo.

- N é número de estrelas da malha grossa.

- $g_{i}, i=1, \ldots, N$, é o grau do vértice central da estrela $i$ da malha grossa.

- $O_{i a_{j}} \in\{0,1,2\}, i=1, \ldots, N, j=1, \ldots, g_{i}$, é a orientação de cada triângulo $a_{j}$ da malha grossa em relação à estrela do vértice $i$. Para cada triângulo $a_{j}$, suas halfedges são numeradas de 0 a 2 . A halfedge de número 0 é a que tem o vértice $i$ como base. As halfedges de números 1 e 2 são as seguintes, em sentido antihorário, a partir da halfedge de número 0. Considerando todas as halfedges da estrela de vértice central $i$ numeradas desta maneira, $O_{i a_{j}}$ é definido como sendo o valor desta numeração para a halfedge que tem como base o vértice $i$ e como a ponta $a_{j-s i m o}$ é vértice da estrela. O primeiro vértice da estrela é definido no arquivo boff-nome.boff (veja a seguir). Os demais vértices são os seguintes ao primeiro, em sentido anti-horário. A Figura 3.4 representa a malha de duas estrelas, com vértices centrais $\alpha$ e $\beta$, com a orientação de cada triângulo $a_{j}$ da malha grossa. Assim, para a Figura 3.4, o conjunto das orientações $O_{i a_{j}}$ da estrela do vértice $\alpha$ começando pelo triângulo 1 é $\{0,0,0,0,0\}$ e o conjunto das orientações $O_{i a_{j}}$ da estrela do vértice $\beta$ começando pelo triângulo 2 é $\{1,2,0,1,2,0,0\}$.

- $n v_{i j}, n f_{i j}, n e_{i j}, i=1, \ldots, N$ e $j=1, \ldots, g_{i}$, são os números de vértices, faces e arestas da malha fina, respectivamente, que estão contidos no triângulo $a_{j}$ da estrela de $i$. 


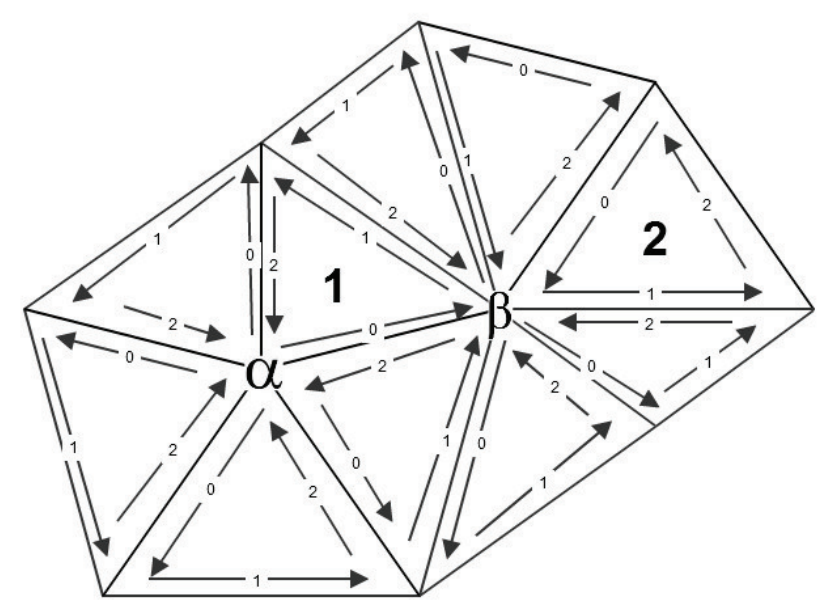

Figura 3.4: Exemplo da orientação de cada estrela da malha grossa.

- $x_{i j k}, y_{i j k}, z_{i j k}, i=1, \ldots, N, j=1, \ldots, g_{i}$ e $k=1, \ldots, n v_{i j}$, são as coordenadas em $\mathbb{R}^{3}$ dos vértices da malha fina que estão contidos no triângulo $a_{j}$ da estrela do vértice $i$;

- $t_{i j k}, i=1, \ldots, N, j=1, \ldots, g_{i}$ e $k=1, \ldots, n v_{i j}$, é o parâmetro que diz se o vértice da malha fina com coordenadas $\left(x_{i j k}, y_{i j k}, z_{i j k}\right)$, que denotaremos por $v$, está na borda, no interior ou é vértice do triângulo $a_{j}$ da malha grossa. Para cada vértice do triângulo $a_{j}$, que denotaremos por $\alpha, \beta$ ou $\gamma$, atribuímos os valores 0,1 ou 2 . O vértice 0 é o vértice central da estrela $i$, digamos $\alpha$, e os vértices 1 e 2 são os vértices do triângulo $a_{j}$ seguintes ao vértice central, seguindo a orientação do triângulo (digamos $\beta$ e $\gamma$ ). Quando o vértice $v$ da malha fina está em um dos vértices do triângulo $a_{j}$, $t_{i j k}$ assume o valor atribuído ao vértice do triângulo. Quando $v$ está no interior do triângulo $a_{j}, t_{i j k}$ assume o valor -1 . Quando $v$ está sobre as retas $\alpha \beta, \beta \gamma$ ou $\gamma \alpha$, $t_{i j k}$ assume valores em $] 0,1[] 1,,2[$ ou $] 2,3[$, respectivamente. A Figura 3.5 ilustra a atribuição dos valores $t_{i j k}$.

- $u_{i j k}, v_{i j k}, i=1, \ldots, N, j=1, \ldots, g_{i}$ e $k=1, \ldots, n v_{i j}$, são as coordenadas baricêntricas do vértice da malha fina em relação ao triângulo $a_{j}$ da malha grossa. Note que são 3 as coordenadas baricêntricas, mas, a partir de duas delas, podemos facilmente calcular a terceira (dada por $\left.1-u_{i j k}-v_{i j k}\right)$.

- $n c_{i j k}, i=1, \ldots, N, j=1, \ldots, g_{i}$ e $k=1, \ldots, n v_{i j}$, é o número que representa o tipo de célula. Para malhas de triângulos, $n c_{i j k}=3$. Para malhas de quadriláteros, $n c_{i j k}=4$, e assim por diante. Consideramos aqui apenas malhas triangulares.

- $b_{i j k}^{1}, b_{i j k}^{2}, b_{i j k}^{3}, i=1, \ldots, N, j=1, \ldots, g_{i}$ e $k=1, \ldots, n v_{i j}$, são os identificadores dos vértices da malha fina que formam uma face (triângulo) da malha fina que pertence à face $a_{j}$. 


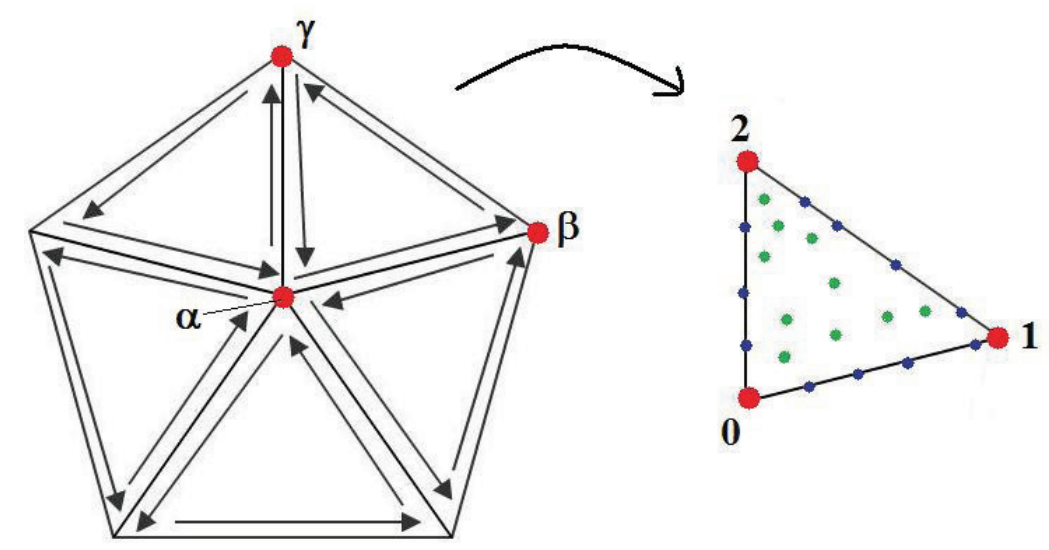

Figura 3.5: Ilustração da atribuição de valores de $t_{i j k}$. Para os pontos vermelhos nos vértices do triângulo, o valor de $t_{i j k}$ é igual ao valor indicado (0,1 ou 2). Para os pontos azuis, $t_{i j k}$ assume valores em $] 0,1[] 1,,2[$ ou $] 2,3\left[\right.$. Para os pontos em verde, $t_{i j k}$ assume o valor -1 .

A Figura 3.6 é um exemplo de um arquivo SOFF contendo informações de uma parte de uma malha real.

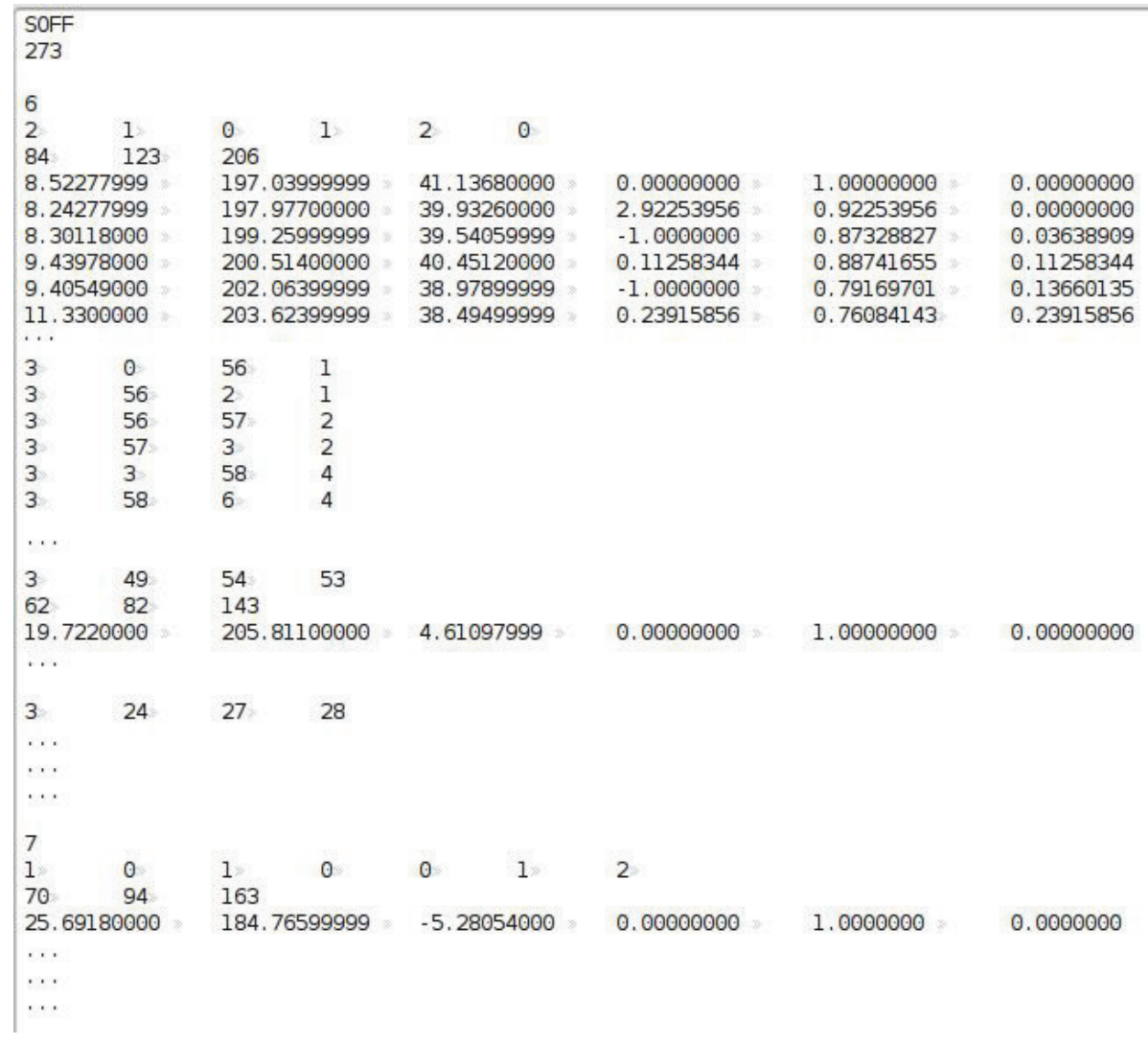

Figura 3.6: Exemplo de arquivo SOFF. 


\section{- boff-nome.boff}

O objetivo deste arquivo é armazenar as informações de um retalho de Bézier (veja Schneider \& Eberly (2003)), para cada estrela da malha grossa. Uma curva de Bézier é uma parametrização construída a partir de um conjunto de pontos, que chamamos pontos de controle, os quais são ponderados por funções polinomiais. Assim como curvas de Bézier, retalhos de Bézier são definidos por um conjunto de pontos de controle. A Figura 3.7 representa um retalho de Bézier com grau 3. A Figura 3.8 representa um retalho de Bézier de grau 9 sobre uma estrela.

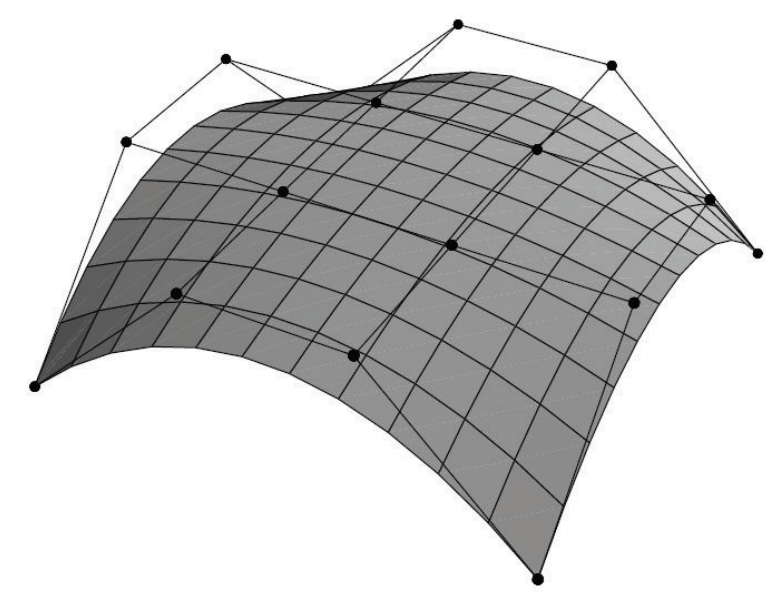

Figura 3.7: Retalho de Bézier (Schneider \& Eberly (2003)).

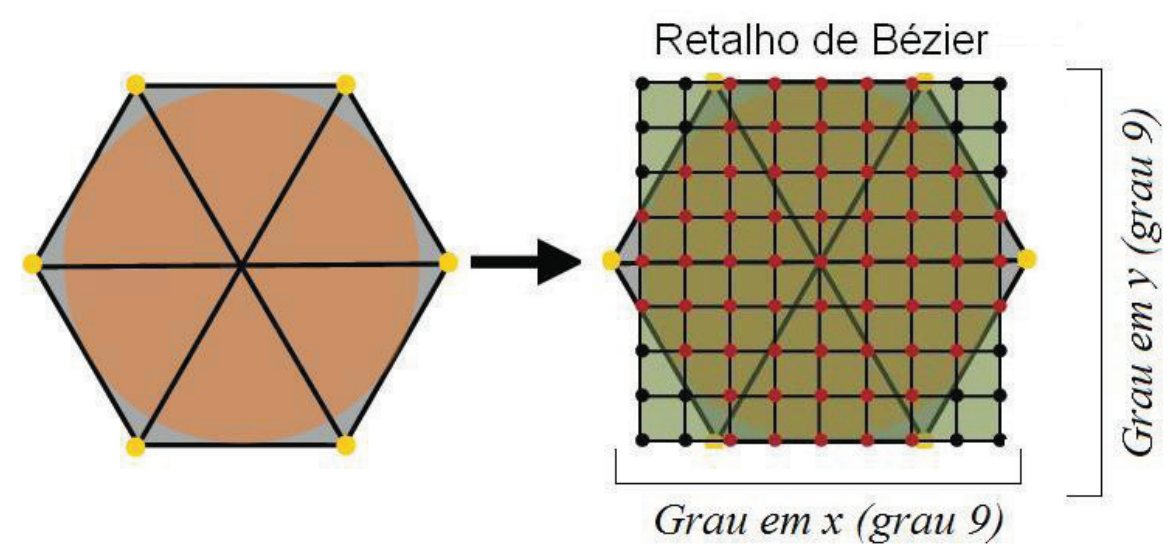

Figura 3.8: Exemplo de um retalho de Bézier. 
O esquema abaixo apresenta o formato de um arquivo BOFF.

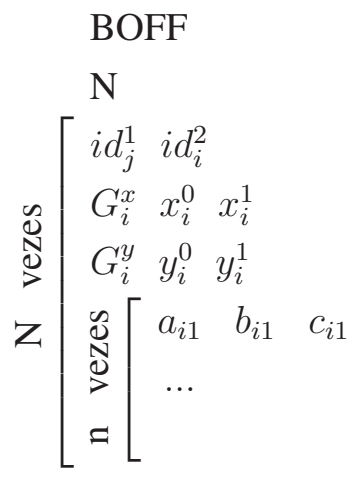

Descrevemos aqui seus detalhes:

- BOFF indica a extensão do arquivo.

- N é número de estrelas da malha grossa.

- $i d_{i}^{1}, i=1, \ldots, N$, é o número de identificação do vértice central da estrela $i$.

- $i d_{i}^{2}, i=1, \ldots, N$, é o número de identificação do vértice para qual a aresta inicial aponta.

- $G_{i}^{x}, i=1, \ldots, N$, é o grau na direção do eixo $x$.

- $x_{i}^{0}, x_{i}^{1}, i=1, \ldots, N$, são os valores inicial e final no eixo $x$.

- $G_{i}^{y}, i=1, \ldots, N$, é o grau na direção do eixo $y$

- $y_{i}^{0}, y_{i}^{1}, i=1, \ldots, N$, são os valores inicial e final no eixo $y$.

- $a_{i j}, b_{i j}, c_{i j}, i=1, \ldots, N, j=1, \ldots,\left(G_{j}^{x}+1\right) \times\left(G_{j}^{y}+1\right)$, são as coordenadas dos pontos de controle da estrela de $i$.

A Figura 3.9 é um exemplo de um arquivo BOFF contendo informações de uma parte de uma malha real. Note que, como estamos tratando de uma parametrização em polígonos regulares, os retalhos de Bézier são quadrados. Assim, $G_{1}=G_{2}, x_{0}=y_{0}, x_{1}=y_{1}$, $x_{0}=-x_{1}$ e $y_{0}=-y_{1}$.

Vale observar que os arquivos BOFF e SOFF são arquivos "irmãos", ou seja, um complementa o outro. As orientações $O_{i a_{j}}$ do arquivo SOFF são construídas a partir de um triângulo inicial, dado pelo $i d_{i}^{2}$ do arquivo BOFF. Em ambos arquivos, o número de estrelas (vértices) $\mathrm{N}$, da malha grossa, são iguais.

O arquivo de saída o-nome.vtk contém a nova malha grossa, gerada usando o processo de otimização descrito no Capítulo 2. Este arquivo tem o formato VTK².

\footnotetext{
${ }^{2}$ VTK, User's Guide, encontrado em www.kitware.com
} 


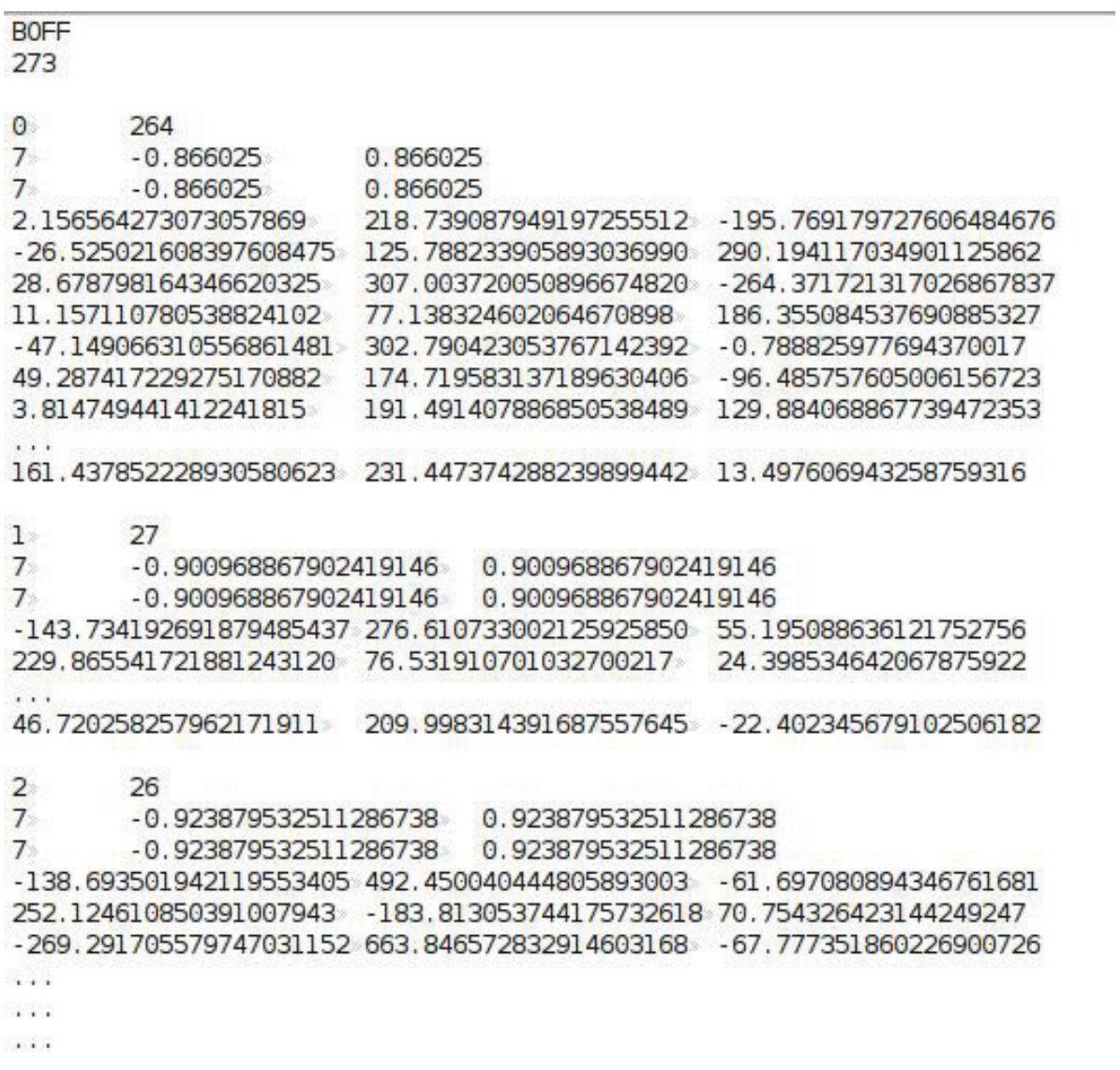

Figura 3.9: Exemplo de arquivo BOFF.

\subsection{Método para otimização de malhas}

Implementamos, em C++, as estruturas descritas nesta seção, bem como o algoritmo descrito no Capítulo 2. Chamamos nossa implementação de MinDistortion.

Descrevemos nesta seção alguns detalhes do algoritmo implementado em MinDistortion, no qual lemos, otimizamos e atualizamos a malha. Inicialmente, precisamos informar ao programa dos arquivos de entrada (f-nome.off, s-nome.off, soff-nome.soff, boff-nome.boff) e o arquivo de saída (o-nome.vtk), descritos na Seção 3.2, nesta ordem. O Algoritmo 7 descreve alguns detalhes dos passos implementados em MinDistortion, que foram previamente mecionados no Capítulo 2. 
Algoritmo 7 Algoritmo implementado no MinDistortion

Passo 1. Leia os arquivos de entrada, f-nome.off, s-nome.off, soff-nome.soff e boff-nome.boff, e contrua as estruturas de dados que armazenam as malhas grossa e fina (como definido nas Seções 3.1 e 3.2).

Passo 2. Atualize a parametrização da malha fina conforme descrito em PPS (Siqueira (2009)).

Passo 3. Defina $s$ Ciclos como o número de vezes que a malha grossa será atualizada com base na otimização da malha fina. Para $i$ de 1 à $s$ Ciclos, repita:

Passo 3.1. Para cada vértice $s v$ da malha grossa, faça:

Passo 3.1.1. Defina uma malha auxiliar fMalha com todos os vértices, arestas e faces da malha fina contidos na estrela da malha grossa e centrada em $s v$.

Passo 3.1.2. Defina um conjunto $V$ com todos os vértices da malha auxiliar fMalha.

Passo 3.1.3. Para cada vértice $f v \in V$, faça:

Passo 3.1.3.1. Considere a estrela E de fMalha centrada em $f v$.

Passo 3.1.3.2. Usando as coordenadas paramétricas dos vértices em $\mathrm{E}$, resolva o problema (2.11).

Passo 3.1.3.3. Atualize as coordenadas paramétricas de $f v$ com a solução do problema (2.11) calculada no Passo 3.1.3.2.

Passo 3.1.3.4. Atualize as coordenadas tridimensionais de $f v$ em $\mathrm{fMalha}$.

Passo 3.1.4. Atualize $s v$ na malha grossa, de acordo com sua posição correspondente em fMalha.

Passo 4. Gere o arquivo o-nome.vtk com a representação da estrutura de dados da malha grossa, usando o formato VTK.

No Passo 3.1.4 do Algoritmo 7, atualizamos o vértice $s v$ na malha grossa, a partir da otimização ocorrida na malha fina. Inicialmente, o vértice $s v$ da malha grossa possui um correspondente $f v$ na malha fina. Com a otimização da parametrização, esse vértice $f v$ é movido. Buscamos, então, a nova localização deste vértice, e, assim, movimentamos o vértice $s v$ de acordo com o vértice $f v$.

Lembramos que cada vértice $f v$ da malha fina possui coordenadas baricêntricas de acordo com os triângulos da malha grossa. Conforme a otimização ocorre, os vértices $f v$ se movimentam na parametrização, mudando de coordenadas, o que implica em recalcular as coordenadas baricêntricas destes vértices. Assim, um vértice da malha fina, ao final da otimização em cada estrela da malha grossa, poderá ter mudado de triângulo, mudando assim suas coordenadas.

A PPS ${ }^{3}$ (Parametric Pseudo-Surfaces for Triangle Meshes) é uma biblioteca livre que permite calcular a parametrização, as coordenadas baricêntricas, e gerar os arquivos BOFF e SOFF. As principais caracteristicas da PPS estão descritas em (Siqueira (2009)).

\footnotetext{
${ }^{3}$ http://www.dimap.ufrn.br/mfsiqueira/Marcelo_Siqueiras_Web_Spot/Software.html
} 
Para resolver os problemas de programação não-linear do Passo 3.1.3.2 do Algoritmo 7, usamos Algencan (veja Capítulo 1). Transformamos AlgEnCAN em uma rotina e implementamos uma interface entre sua implementação em Fortran 77 com nosso código em C++. Todos os parâmetros padrão foram utilizados.

No próximo capítulo, apresentamos os modelos de malhas utilizados e os resultados numéricos obtidos na otimização de malhas. 



\section{Resultados numéricos}

Neste capítulo apresentamos os resultados obtidos na aplicação de MinDistortion para a otimização de malhas triangulares de superfícies no $\mathbb{R}^{3}$.

\subsection{Modelos de malhas utilizadas}

Para verificar o comportamento e desempenho do MinDistortion, método de otimização de malhas implementado em C++ e descrito no Capítulo 3, utilizamos duas malhas (finas e grossas) de modelos utilizados na literatura: Bimba Con Nastrino e Botijo.

A malha Bimba Con Nastrino é um modelo que contém 72 vértices na malha grossa e 14.839 vértices na malha fina. As Figuras 4.1 e 4.2 representam as malhas fina e grossa do modelo Bimba Con Nastrino.

A malha Botijo é um modelo que contém 273 vértices na malha grossa e 20.000 vértices na malha fina. As Figuras 4.3 e 4.4 representam as malhas fina e grossa do modelo Botijo.

\subsection{Experimentos computacionais}

Todos os experimentos foram realizados utilizando um computador com processador Intel Core 2 Duo de 2.66GHz, com 3.0GB de memória RAM e sistema operacional Linux de 64 bits (Ubuntu 10.04). Os códigos foram compilados com g++ (GCC 4.4.3). Utilizamos dois critérios de parada: tempo de execução e número de iterações $s$ Ciclos. Utilizamos o tempo máximo de 1 hora e o número de iterações variou de entre os valores 1, 5, 10, 25 e 50. A Tabela 4.1 


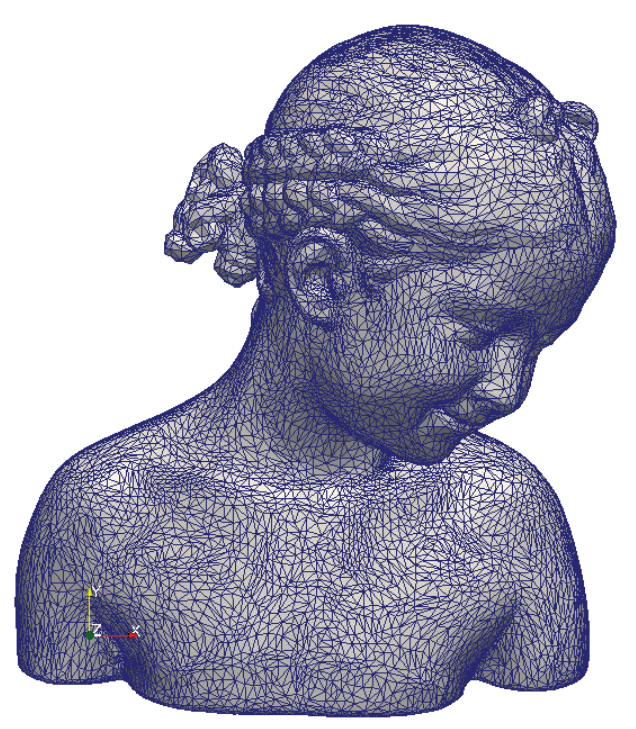

Figura 4.1: Malha fina do modelo Bimba Con Nastrino.

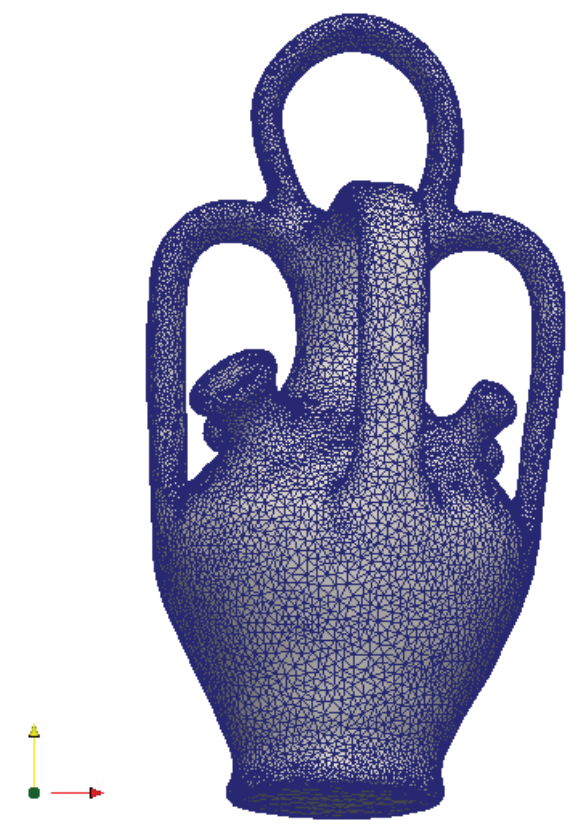

Figura 4.3: Malha fina do modelo Botijo.

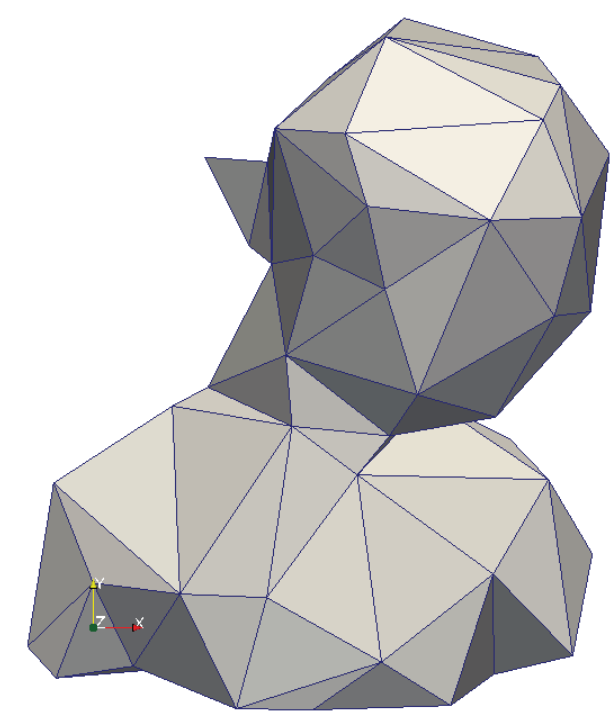

Figura 4.2: Malha grossa do modelo Bimba Con Nastrino.

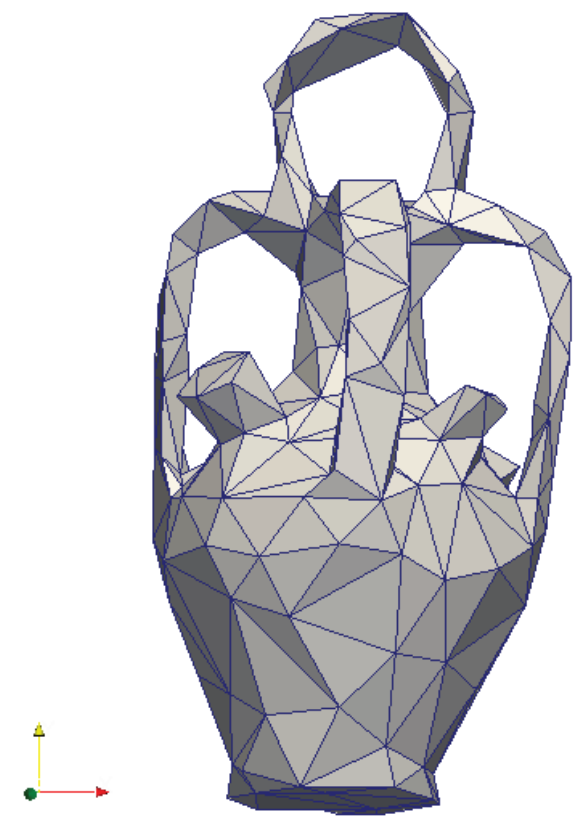

Figura 4.4: Malha grossa do modelo Botijo.

mostra o tempo, em segundos, utilizado para a execução do MinDistortion na otimização de cada uma das malhas, para cada valor diferente de número de iterações. Note que a imposição de tempo limite de execução impediu que fossem realizadas 50 iterações para o modelo Bimba Con Nastrino. 


\begin{tabular}{|c|c|c|c|c|c|}
\hline Número de iterações & 1 & 5 & 10 & 25 & 50 \\
\hline \hline Bimba Con Nastrino & 68.15 & 487.57 & 972.23 & 3242.37 & - \\
\hline Botijo & 49.53 & 257.15 & 518.04 & 1286.12 & 3003.87 \\
\hline
\end{tabular}

Tabela 4.1: Tempo de execução, em segundos

\subsection{Critério de qualificação}

Para quantificar a qualidade da otimização realizada, utilizamos dois critérios diferentes. Analisamos a distorção que a parametrização cometeu no ângulo e na área de cada triângulo da malha fina separadamente, antes e após a otimização.

\subsubsection{Distorção angular}

Para analisar a distorção angular, percorremos todos triângulos da malha fina na superfície e computamos os ângulos de cada triângulo. Em seguida, percorremos cada triângulo da malha fina na parametrização e computamos os ângulos equivalentes aos da malha fina na superfície. Posteriormente, calculamos a razão entre estes dois valores. Com os valores da razão angular de cada triângulo da malha fina, construímos um histograma que nos permite observar a distorção ocorrida na malha fina original após a parametrização. O mesmo procedimento foi realizado para a malha fina na superfície e parametrizada após a otimização.

Apresentamos agora as qualificações de ângulo obtidas para cada um dos modelos utilizados.

\subsubsection{Bimba Con Nastrino}

As Figuras 4.5, 4.7 e 4.9 apresentam os histogramas de qualidade da parametrização do modelo Bimba Con Nastrino antes da otimização. As Figuras 4.6, 4.8 e 4.10 apresentam os histogramas de qualidade da parametrização do modelo Bimba Con Nastrino após a otimização, realizada com 25 iterações.

Observe que os histogramas das Figuras 4.6, 4.8 e 4.10, após a otimização, obtiveram maior concentração de valores em torno do valor 1. Isto era esperado, já que buscamos uma parametrização onde os ângulos fossem preservados.

\subsubsection{Botijo}

As Figuras 4.11, 4.13 e 4.15 apresentam os histogramas de qualidade da parametrização do modelo Botijo antes da otimização. As Figuras 4.12, 4.14 e 4.16 apresentam os histogramas de qualidade da parametrização do modelo Botijo após a otimização, realizada com 50 iterações. 


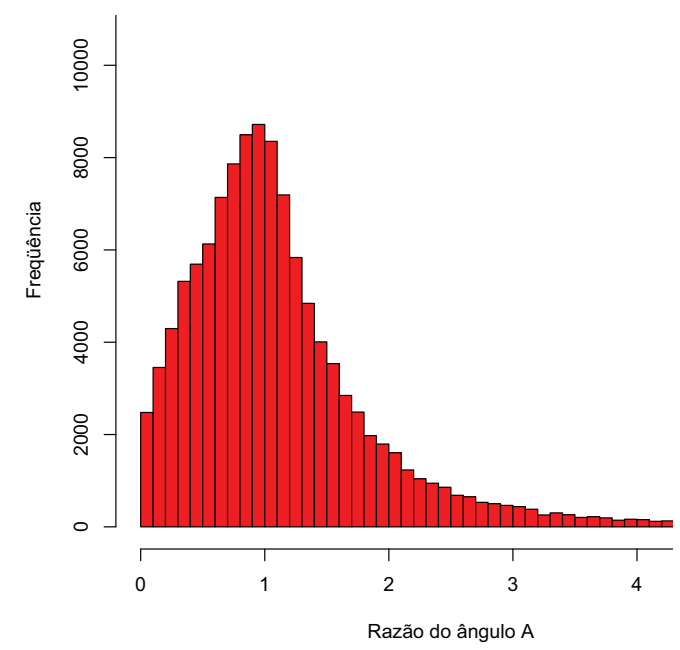

Figura 4.5: Histograma de qualidade antes da otimização do ângulo A.

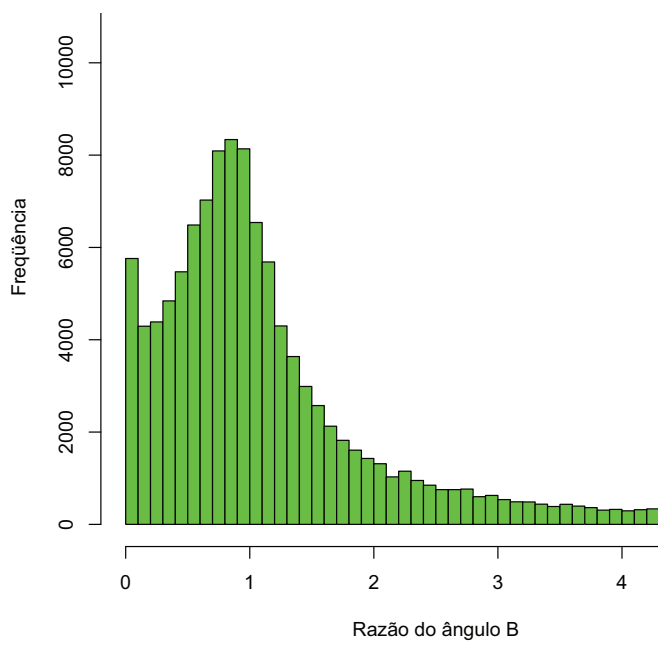

Figura 4.7: Histograma de qualidade antes da otimização do ângulo B.

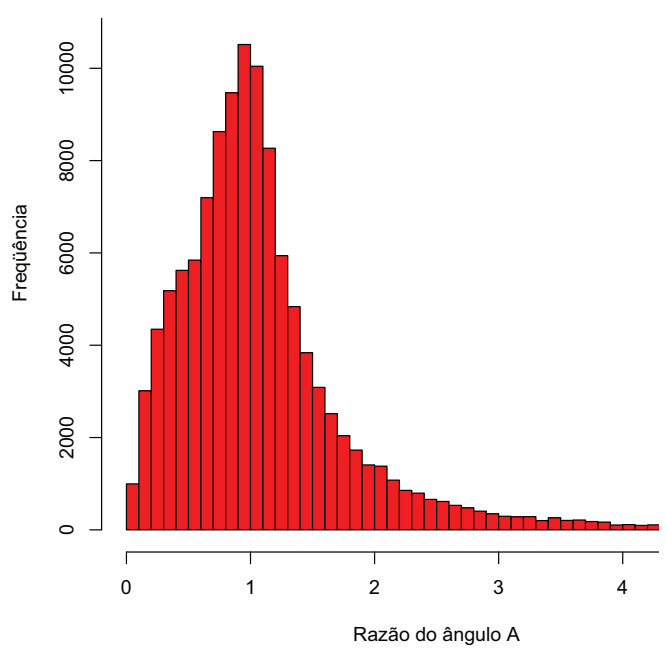

Figura 4.6: Histograma de qualidade após a otimização do ângulo A.

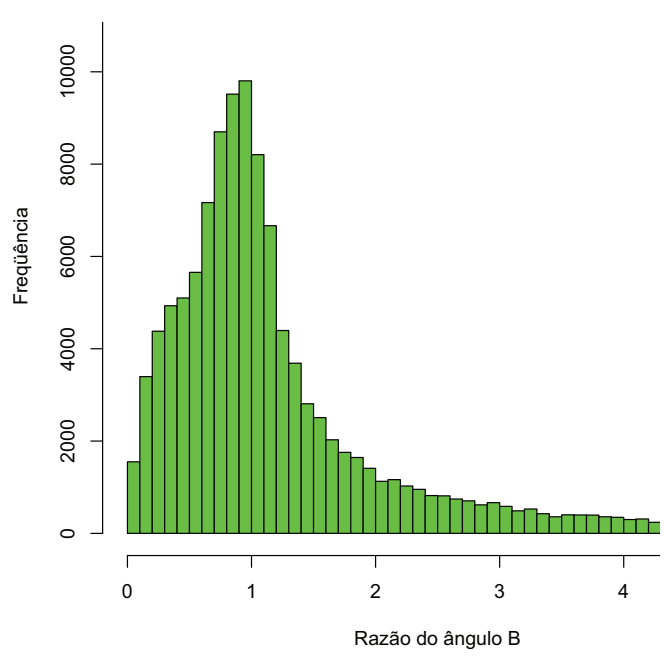

Figura 4.8: Histograma de qualidade após a otimização do ângulo B. 


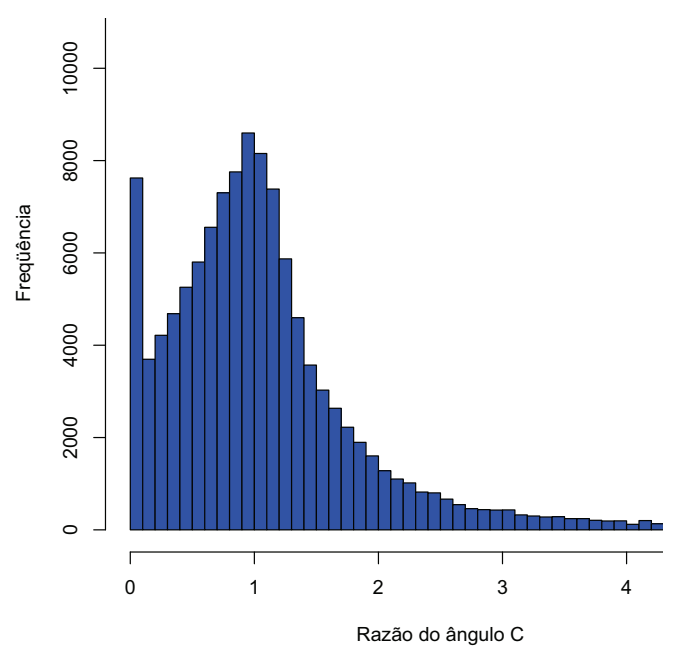

Figura 4.9: Histograma de qualidade antes da otimização do ângulo C.

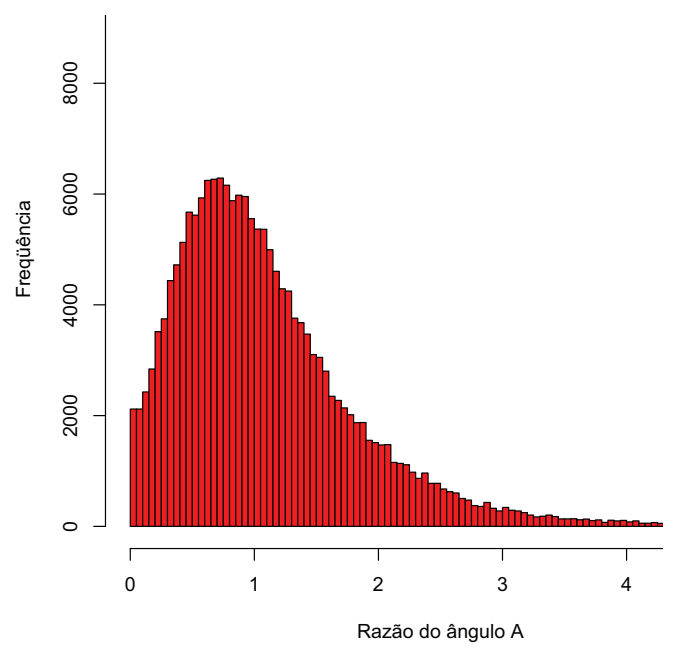

Figura 4.11: Histograma de qualidade antes da otimização do ângulo A.

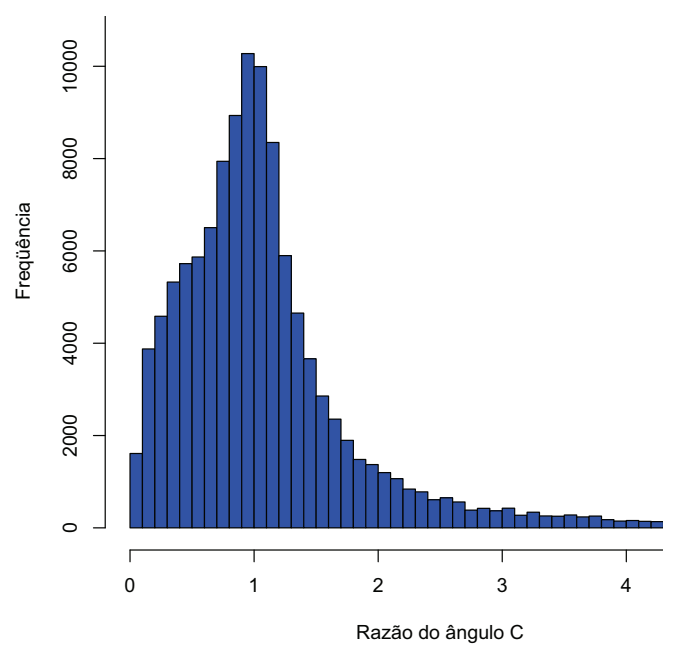

Figura 4.10: Histograma de qualidade após a otimização do ângulo C.

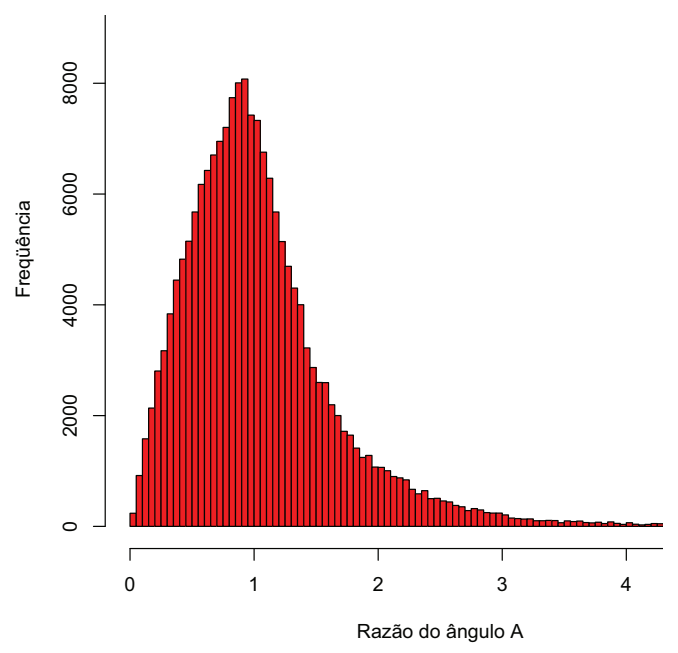

Figura 4.12: Histograma de qualidade após a otimização do ângulo A. 


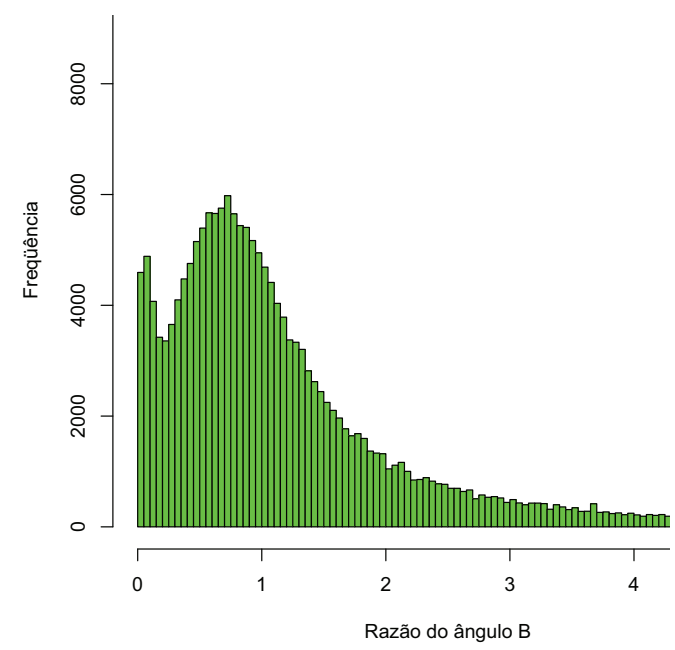

Figura 4.13: Histograma de qualidade antes da otimização do ângulo B.

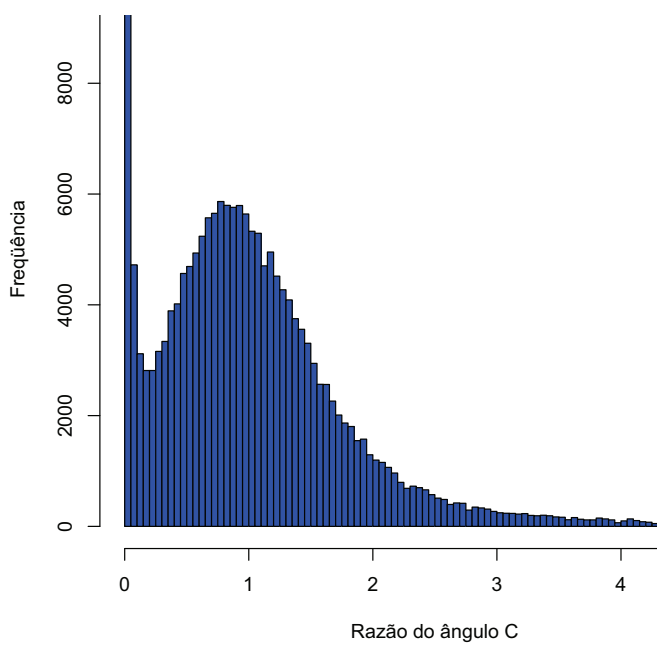

Figura 4.15: Histograma de qualidade antes da otimização do ângulo C.

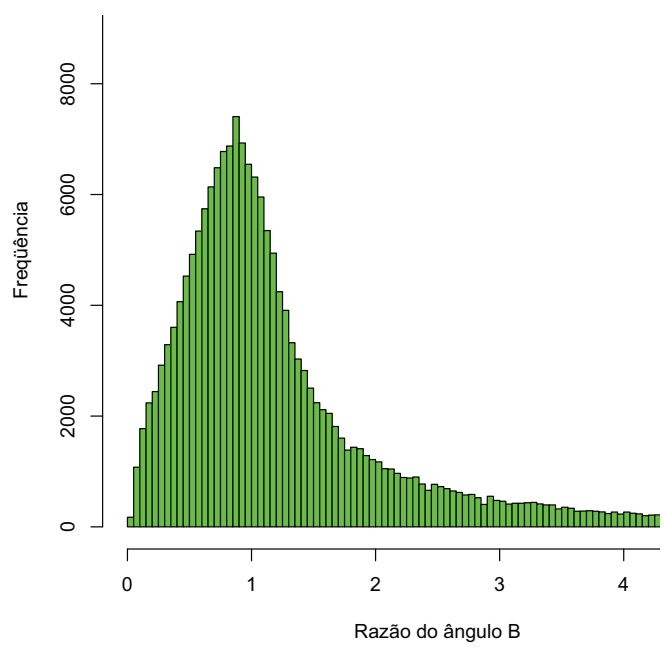

Figura 4.14: Histograma de qualidade após a otimização do ângulo B.

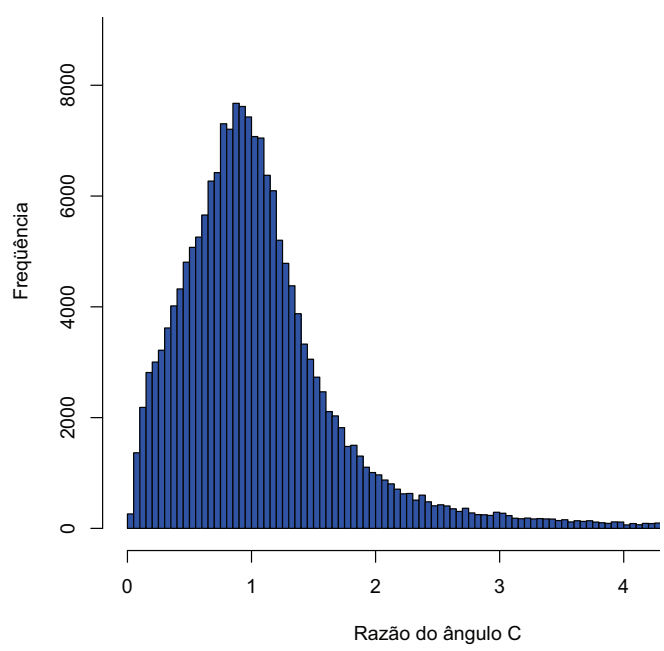

Figura 4.16: Histograma de qualidade após a otimização do ângulo $\mathrm{C}$. 
Como para o modelo Botijo, os histogramas das Figuras 4.12, 4.14 e 4.16, após a otimização, também obtiveram maior concentração de valores em torno do valor 1 .

\subsubsection{Distorção da área}

Para analisar a distorção da área, percorremos todos triângulos da malha fina na superfície e computamos suas áreas. Em seguida, percorremos cada triângulo da malha fina na parametrização e também computamos suas áreas. Posteriormente calculamos a razão entre estes dois valores. Com os valores da razão da área de cada triângulo da malha fina, construímos um histograma que nos permite observar a distorção ocorrida na malha fina original após a parametrização. $\mathrm{O}$ mesmo foi realizado para a malha fina na superfície e parametrizada após a otimização.

Apresentamos agora as qualificações de área obtidas para cada um dos modelos utilizados.

\subsubsection{Bimba Con Nastrino}

A Figura 4.17 apresenta o histograma de qualidade da parametrização do modelo Bimba Con Nastrino antes da otimização. A Figura 4.18 apresenta o histograma de qualidade da parametrização do modelo Bimba Con Nastrino após a otimização, realizada com 25 iterações.

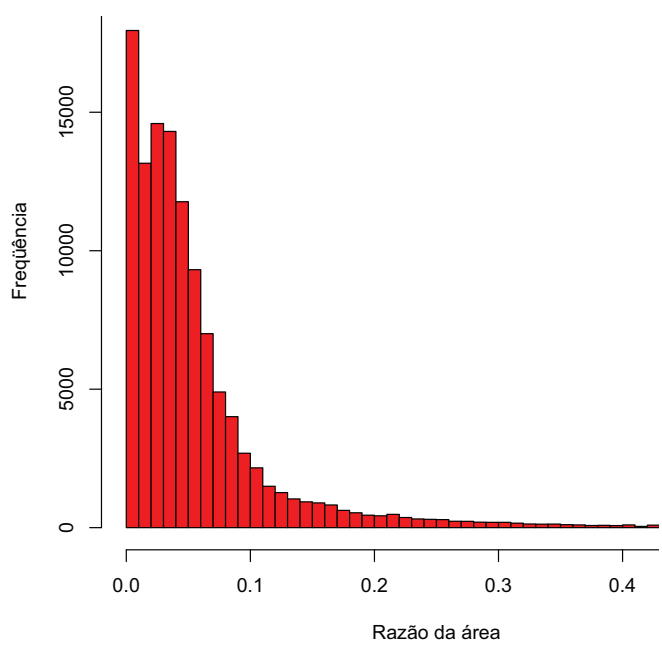

Figura 4.17: Histograma de qualidade antes da otimização.

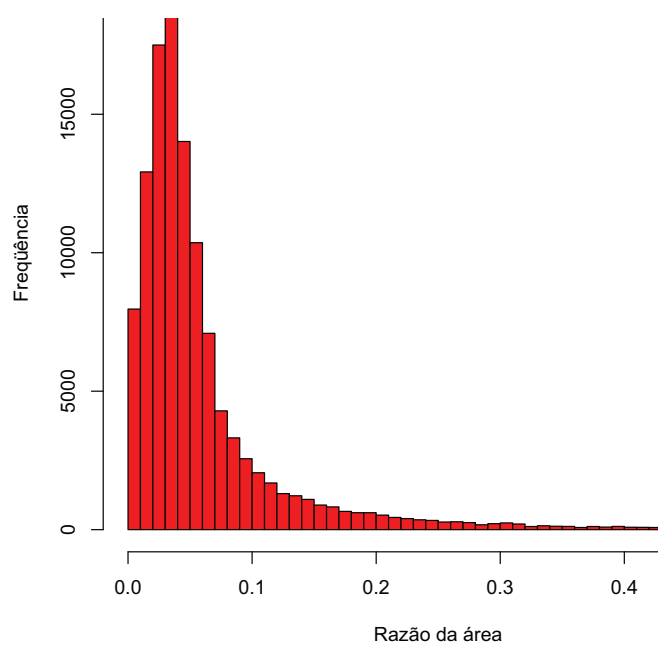

Figura 4.18: Histograma de qualidade após a otimização.

Observando os histogramas das Figuras 4.17 e 4.18, podemos notar que as mudanças ocorridas em relação à área após a otimização são pequenas. 


\subsubsection{Botijo}

A Figura 4.19 apresenta o histograma de qualidade da parametrização do modelo Botijo antes da otimização. A Figura 4.20 apresenta o histograma de qualidade da parametrização do modelo Botijo após a otimização, realizada com 50 iterações.

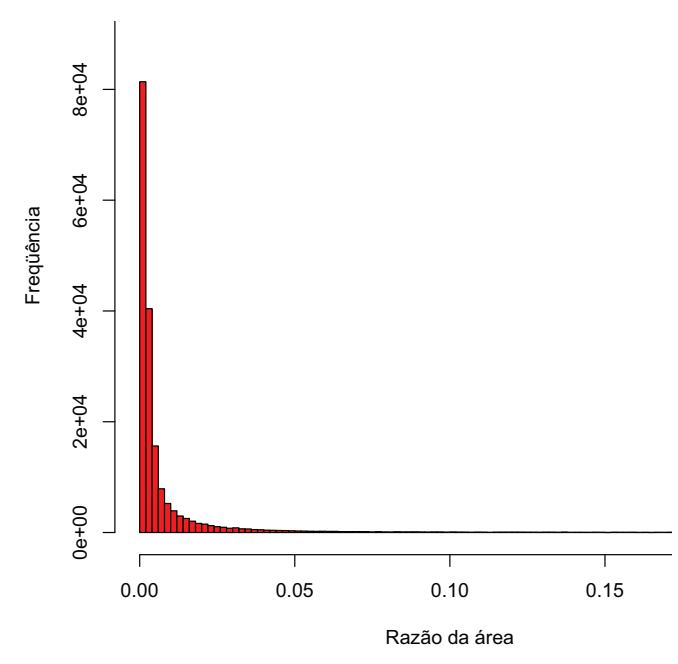

Figura 4.19: Histograma de qualidade antes da otimização.

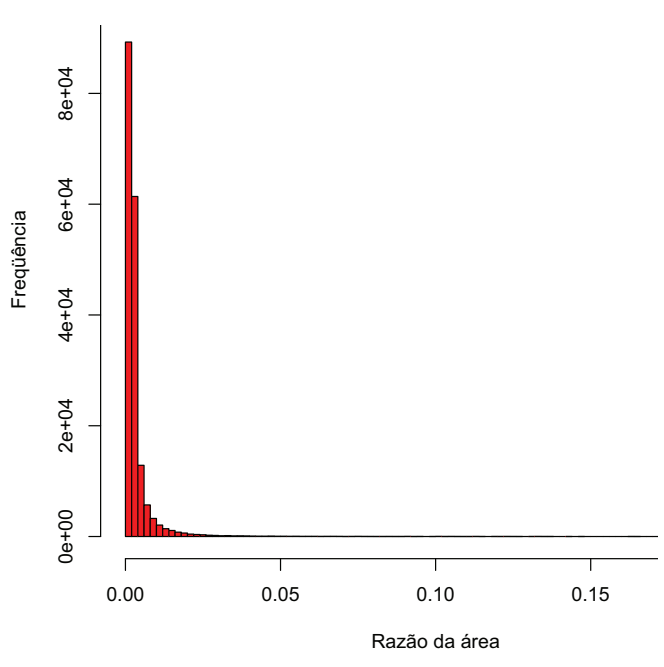

Figura 4.20: Histograma de qualidade após a otimização.

Observando os histogramas das Figuras 4.19 e 4.20, podemos notar que as mudanças ocorridas em relação à área após a otimização são muito pequenas, assim como no modelo Binba Con Nastrino.

\subsection{Consequências da otimização na malha grossa}

Como consequência da minimização das distorções de ângulo e área, podemos observar a melhora dos triângulos da malha grossa de acordo com as iterações feitas. Analisamos aqui o menor ângulo de cada triângulo da malha grossa de cada modelo utilizado.

\subsubsection{Bimba Con Nastrino}

A Figura 4.21 mostra a malha grossa original do modelo Bimba Con Nastrino colorida de acordo com a escala de menores ângulos de cada triângulo. O histograma apresentado na Figura 4.22 nos permite observar como estão distribuídos os triângulos de acordo com seus menores ângulos.

As Figuras 4.23, 4.25, 4.27 e 4.29 apresentam a malha grossa gerada após 1, 5, 10, 25 iterações da otimização e as Figuras 4.24, 4.26, 4.28 e 4.30 apresentam os histogramas gerados a partir das malhas otimizadas. 


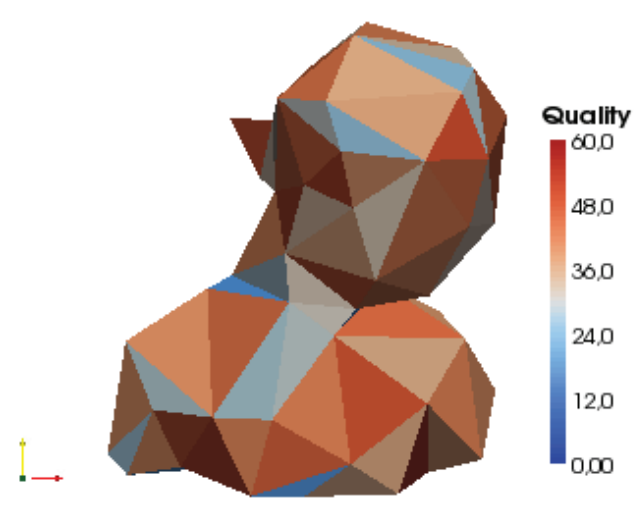

Figura 4.21: Malha grossa original do modelo Bimba Con Nastrino.

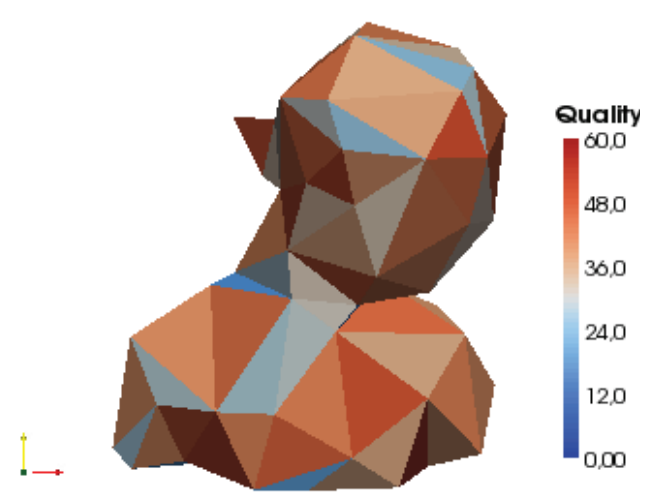

Figura 4.23: Malha grossa do modelo Bimba Con Nastrino após 1 iteração da otimização.

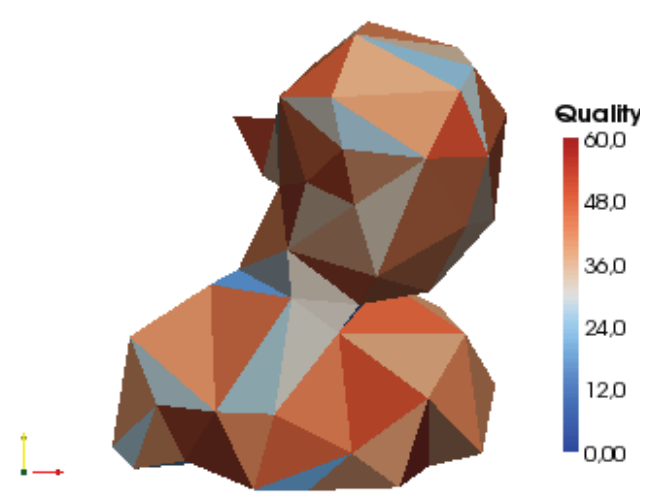

Figura 4.25: Malha grossa do modelo Bimba Con Nastrino após 5 iterações da otimização.

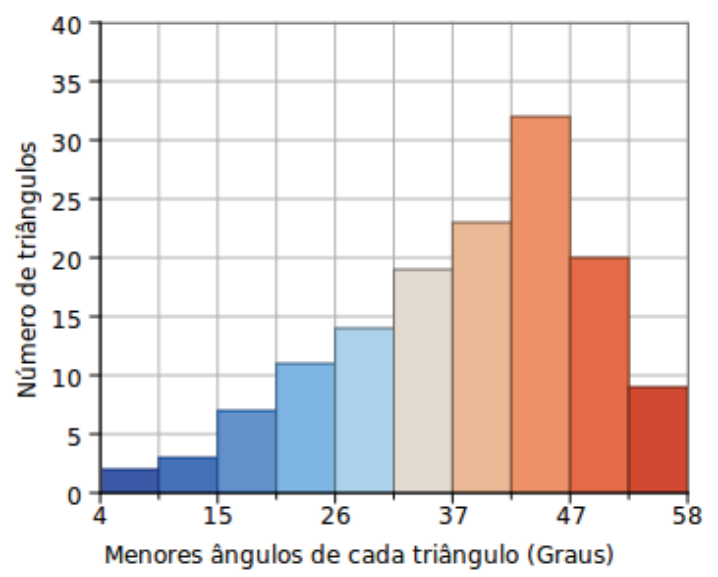

Figura 4.22: Histograma da Figura 4.21.

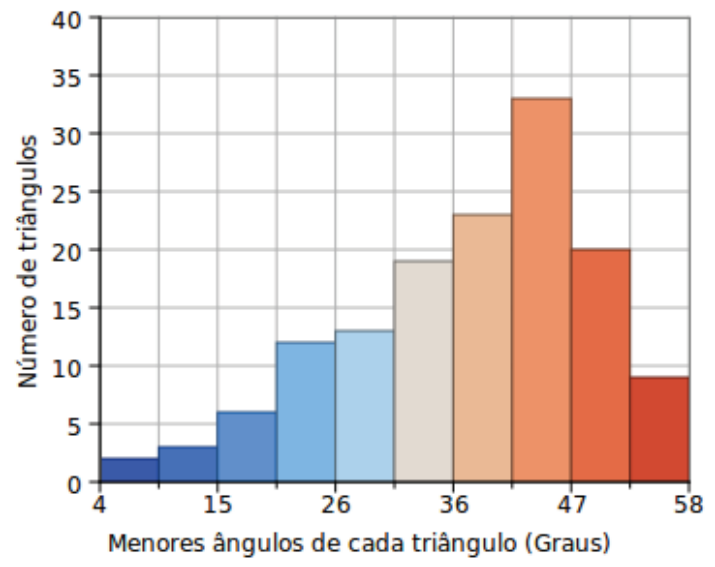

Figura 4.24: Histograma da Figura 4.23.

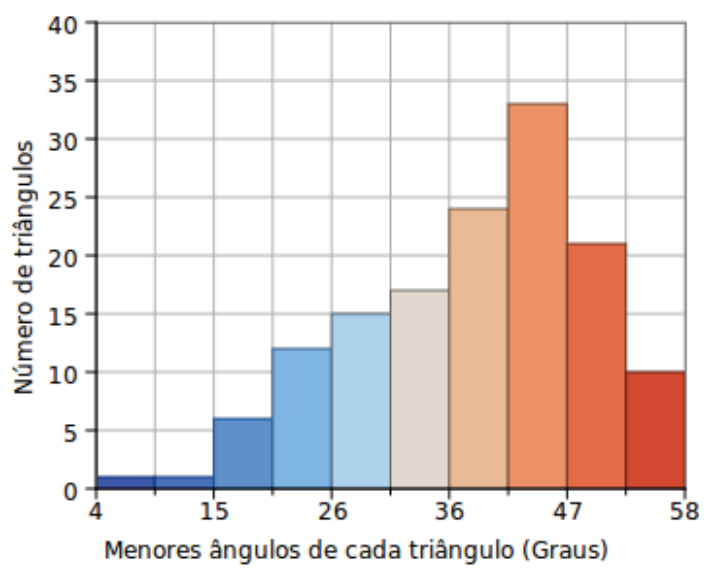

Figura 4.26: Histograma da Figura 4.25. 


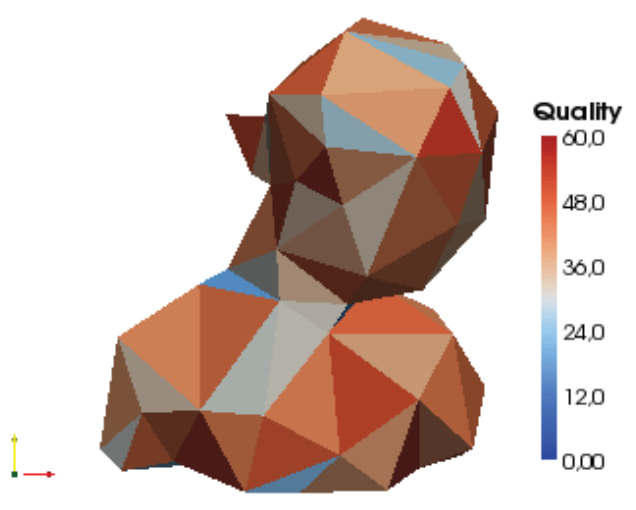

Figura 4.27: Malha grossa do modelo Bimba Con Nastrino após 10 iterações da otimização.

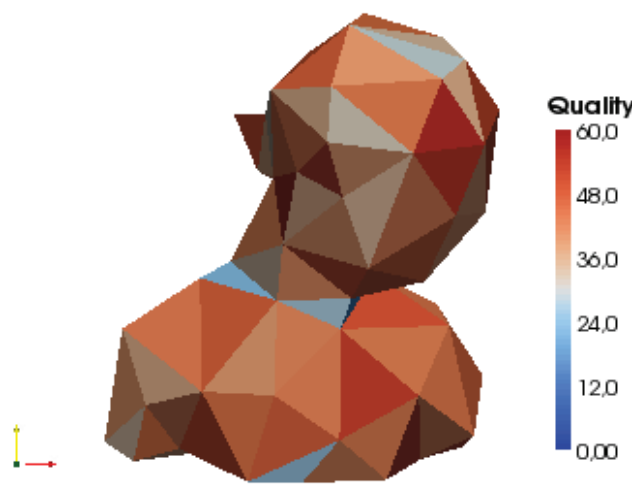

Figura 4.29: Malha grossa do modelo Bimba Con Nastrino após 25 iterações da otimização.

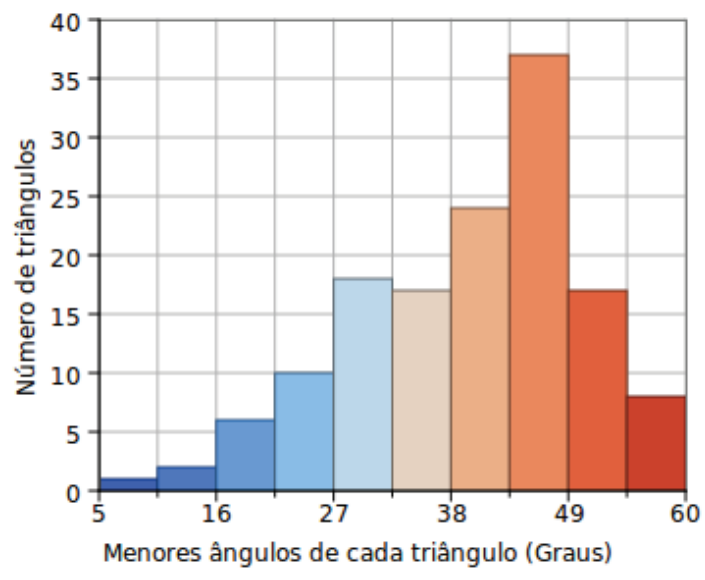

Figura 4.28: Histograma da Figura 4.27.

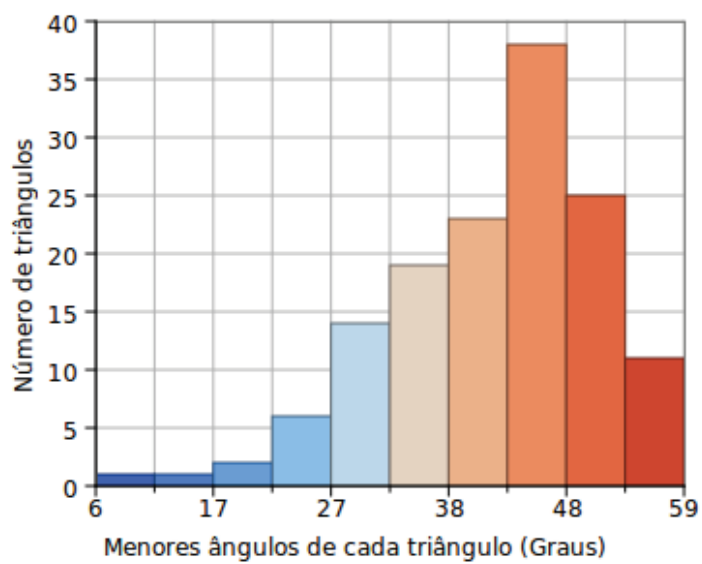

Figura 4.30: Histograma da Figura 4.29. 
Observe que, quanto maior o número de iterações da rotina de otimização, a malha grossa tende a se tornar mais homogênea, ou seja, os triângulos da malha grossa se tornam o mais regular possível.

Note que o histograma apresentado na Figura 4.22 apresenta triângulos com o menor ângulo a partir do valor 4 graus, sendo que no histograma apresentado na Figura 4.30, após a otimização, o menor ângulo computado tem valor 6 graus. Note também que os valores abaixo de 30 graus diminuíram e os valores acima de 30 graus aumentaram após a otimização.

\subsubsection{Botijo}

A Figura 4.31 mostra a malha grossa original do modelo Botijo, colorida de acordo com a escala de menores ângulos de cada triângulo. O histograma apresentado na Figura 4.32 nos permite observar como estão distribuídos os triângulos, de acordo com seus menores ângulos.

As Figuras 4.33, 4.35, 4.37, 4.39 e 4.41 apresentam a malha grossa gerada após 1, 5, 10, 25 e 50 iterações da otimização e as Figuras 4.34, 4.36, 4.38, 4.40 e 4.42 apresentam os histogramas gerados a partir da malha otimizada.

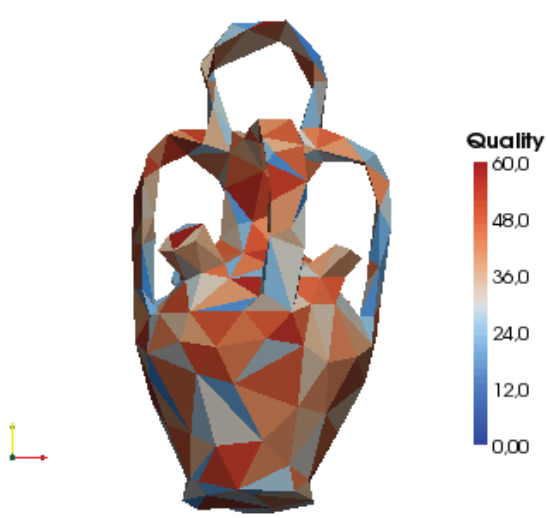

Figura 4.31: Malha grossa original do modelo Botijo.

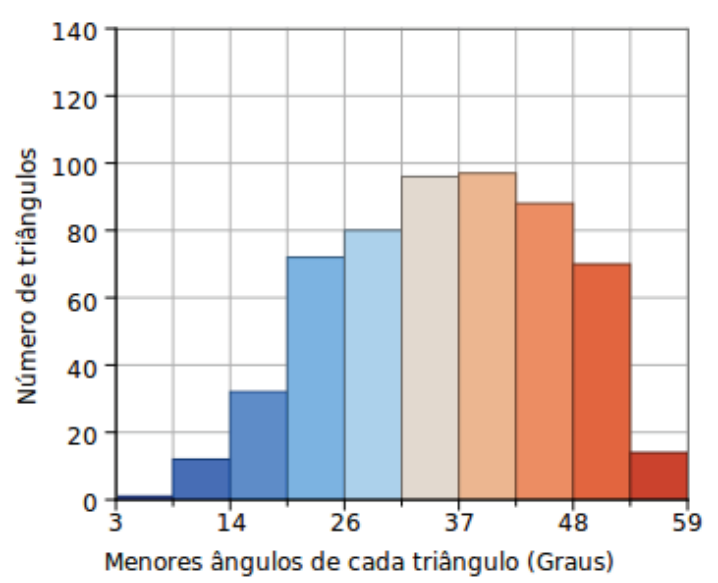

Figura 4.32: Histograma da Figura 4.31.

Note que o histograma apresentado na Figura 4.22 apresenta triângulos com o menor ângulo a partir do valor 3 graus, sendo que, no histograma apresentado na Figura 4.42, após a otimização, o menor ângulo computado tem valor 4 graus. Note também que os valores abaixo de 26 graus diminuíram e os valores acima de 26 graus aumentaram consideravelmente após a otimização.

No capítulo a seguir, apresentamos as conclusões e as sugestões para trabalhos futuros. 


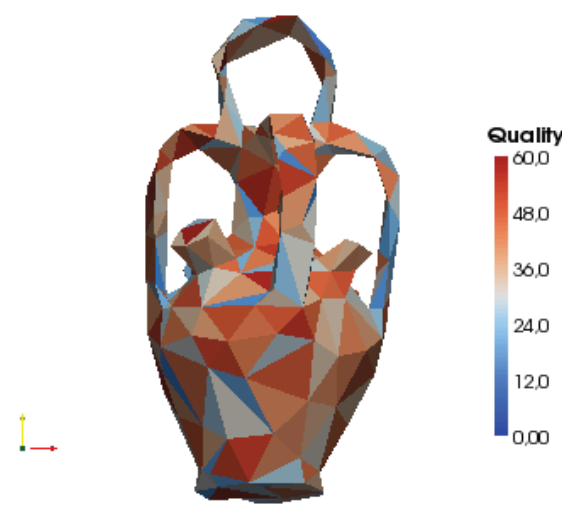

Figura 4.33: Malha grossa do modelo Botijo após 1 iteração da otimização.

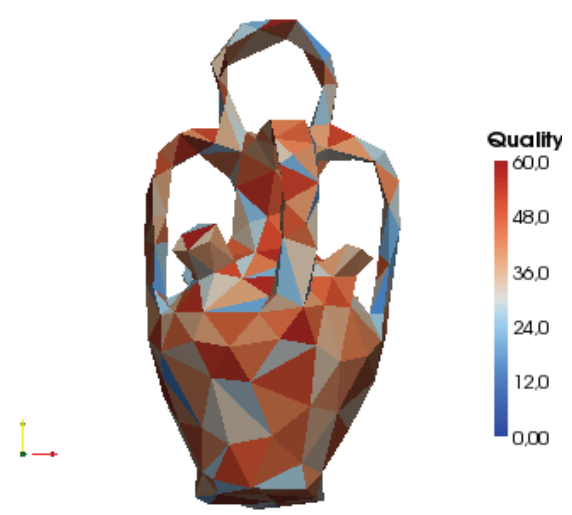

Figura 4.35: Malha grossa do modelo Botijo após 5 iterações da otimização.

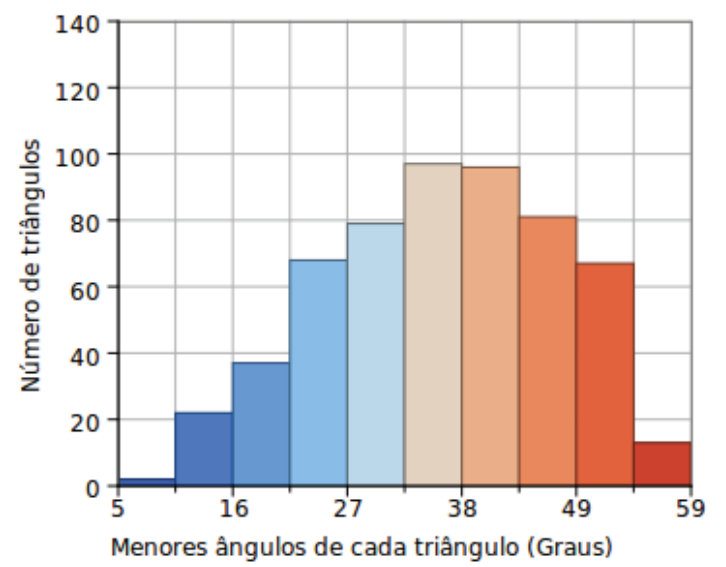

Figura 4.34: Histograma da Figura 4.33.

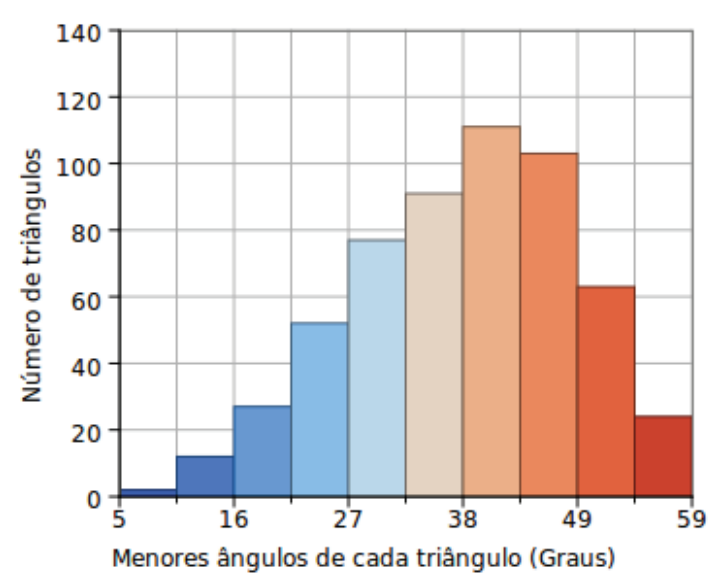

Figura 4.36: Histograma da Figura 4.35. 


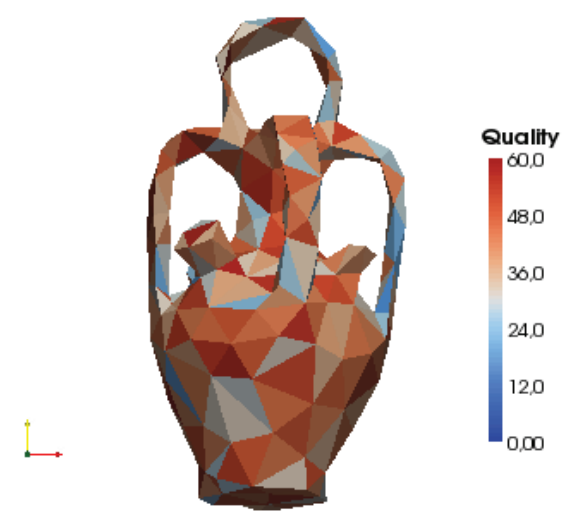

Figura 4.37: Malha grossa do modelo Botijo após 10 iterações da otimização.

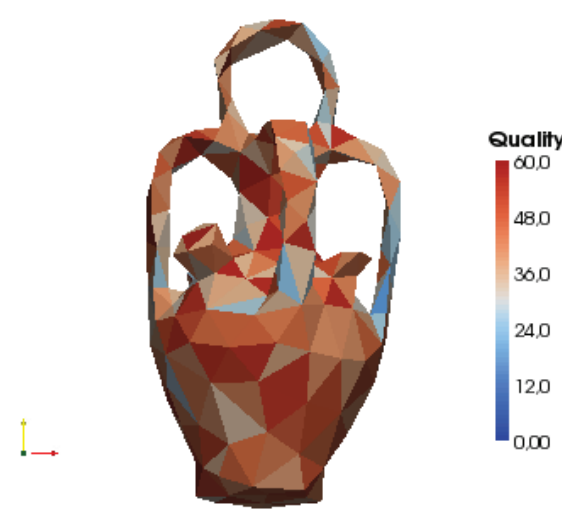

Figura 4.39: Malha grossa do modelo Botijo após 25 iterações da otimização.

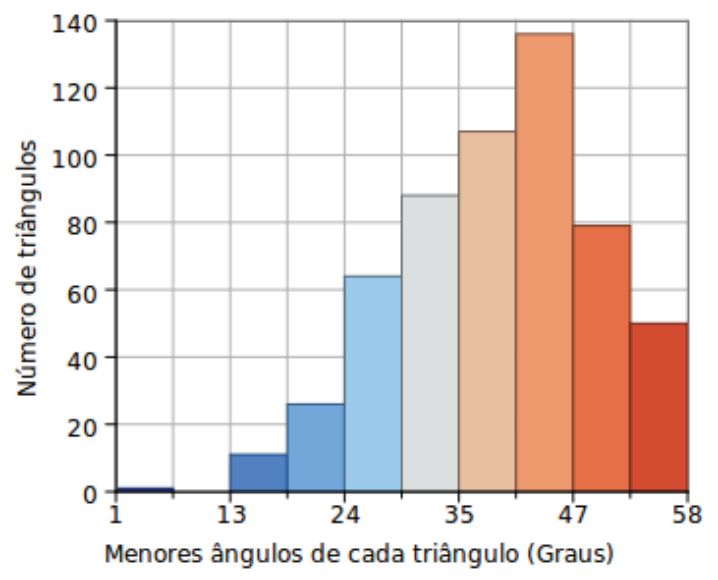

Figura 4.38: Histograma da Figura 4.37.

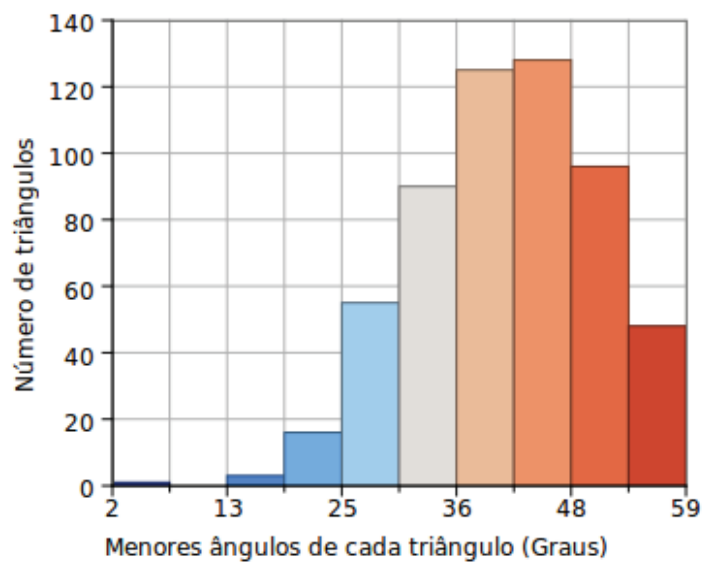

Figura 4.40: Histograma da Figura 4.39. 

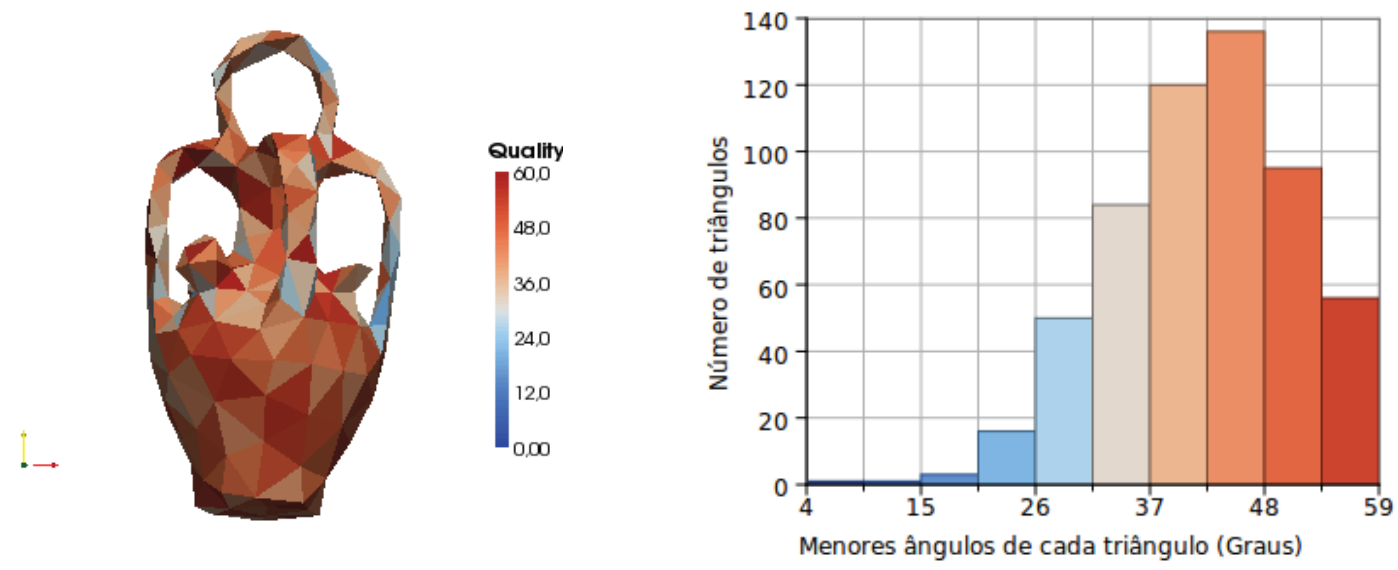

Figura 4.41: Malha grossa do modelo Botijo após 50 iterações da otimização.

Figura 4.42: Histograma da Figura 4.41. 


$\frac{5}{5}$

\section{Conclusões e trabalhos futuros}

Apresentamos um estudo sobre um método de Lagrangianos aumentados implementado em ALGENCAN e observamos seu comportamento quando aplicamos a um problema encontrado na área de Computação Gráfica. O problema estudado e resolvido é um problema encontrado na geração de malhas de superfícies, na etapa de pós-processamento, para o qual propomos uma técnica de otimização visando a melhoria dos elementos da malha.

Quando se trata de geração de malhas de superfícies em $\mathbb{R}^{3}$, parametrizações de malhas triangulares que representam superfícies são usadas em muitas aplicações de processamento de malhas para vários fins. Muitas vezes é necessário preservar a métrica da superfície e, assim, minimizar a deformação do ângulo e da área. A técnica de otimização que propomos tem como objetivo minimizar as distorções de ângulos e áreas impostas por uma parametrização.

Apresentamos um estudo sobre malhas, suas características e definições, e apresentamos um modelo de programação não-linear para o problema em questão. Implementamos uma estrutura de dados baseada halfedge e utilizamos ALGENCAN como subrotina do método implemtado que chamamos de MinDistortion.

O tempo de execução do MinDistortion pode ocasionar problemas quando se busca um método rápido para otimização de malhas, porém percebemos as soluções podem melhorar muito, se o tempo for desconsiderado. Além disso, a implementação ainda pode ser melhorada, ocasionando queda no tempo computacional.

Comparamos as distorções de ângulo e área provocada pela parametrização, antes e depois da otimização. Notamos que, embora a Energia Combinada, descrita no Capítulo 2, leve em consideração a distorção da área, os testes mostram que, na prática, essa distorção não é 
melhorada. Em compensação, a distorção do ângulo é melhorada após a execução da SmoothinSurface. Como consequência da otimização realizada, os triângulos da malha grossa se tornam melhores, considerando o critério de qualidade angular.

Consideramos estes resultados promissores e percebemos a necessidade de novas pesquisas na área. A continuação deste trabalho pode seguir diversas direções, entre elas:

1. Como a minimização da distorção da área é aparentemente desprezada na prática, testes desconsiderando esta distorção podem se mostrar mais eficientes.

2. Espera-se que, ao se considerar as três estrelas da malha grossa que influênciam na parametrização de um mesmo conjunto de vértices da malha fina, o modelo de otimização não-linear se torne mais eficaz na melhoria das malhas.

3. Um próximo passo será estudar a estabilidade e a eficiência de ALGENCAN para resolver este tipo de problema.

4. Um próximo passo será estudar outros métodos de otimização não-linear para resolver o problema (2.11) e comparar a performance da variação do MinDistortion que usam diferentes métodos de otimização não-linear. 


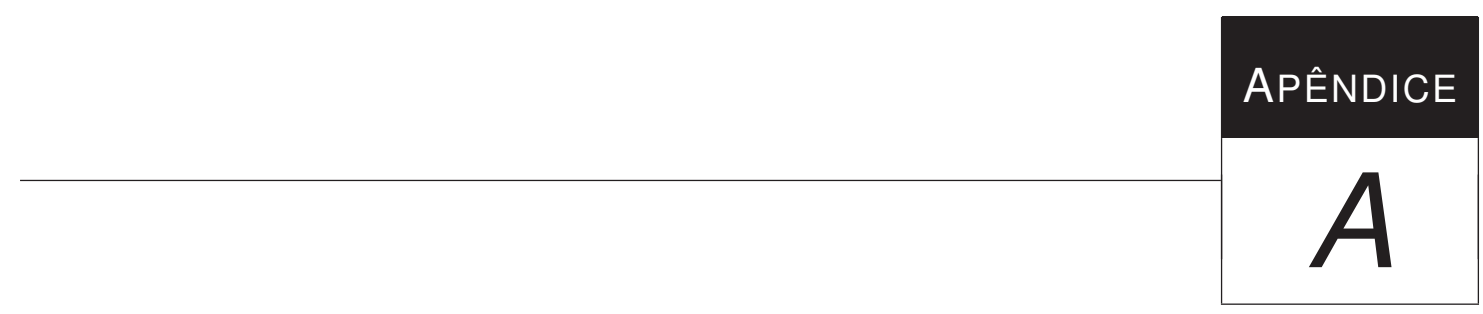

\section{Algoritmos}

Neste apêndice descrevemos alguns algoritmos referentes ao Capítulo 1.

Algoritmo 8 Backtracking com interpolação quadrática uni-dimensional para calcular $\alpha_{k}$

A quadrática interpolante é dada por $q(w), \operatorname{com} q(0)=\bar{f}\left(x_{k}\right), a\left(\alpha_{k}\right)=\bar{f}\left(x_{k}+\alpha_{k} p_{k}\right)$ e $\nabla q(0)=$ $\nabla \bar{f}\left(x_{k}\right)^{T} p_{k}$. Definimos $\alpha_{\text {novo }}$ como um valor tal que $\alpha_{\text {novo }} \leq \alpha_{\max }$ e $\bar{f}\left(x_{k}+\alpha_{\text {novo }} p_{k}\right) \leq$ $\bar{f}\left(x_{k}\right)+\lambda \alpha_{\text {novo }} \nabla \bar{f}\left(x_{k}\right)^{T} p_{k}$.

Seja a iteração atual. Dados $\alpha_{k}$ um tamanho de passo, $p_{k}$ uma direção de descida a partir de $x_{k}$ e $\lambda \in(0,1)$.

Passo 1. Calcule

$$
\alpha_{\max }=\max \left\{\alpha \in[0,1] \mid\left[x_{k}, x_{k}+\alpha p_{k}\right] \subset \Omega\right\} .
$$

Passo 2. Faça $\alpha_{\text {tent }} \leftarrow \alpha_{\max }$.

Passo 3. Se

$$
\bar{f}\left(x_{k}+\alpha_{\text {tent }} p_{k}\right) \leq \bar{f}\left(x_{k}\right)+\gamma \alpha_{\text {tent }} \nabla \bar{f}\left(x_{k}\right)^{T} p_{k}
$$

então faça $\alpha_{k}=\alpha_{\text {tent }}$ e PARE.

Passo 4. Calcule

$$
\alpha_{\text {novo }}=-\frac{\alpha_{\text {tent }}^{2} \nabla \bar{f}\left(x_{k}\right)^{T} p_{k}}{2\left(\bar{f}\left(x_{k}+\alpha_{\text {tent }} p_{k}\right)-\bar{f}\left(x_{k}\right)-\alpha_{\text {tent }} \nabla \bar{f}\left(x_{k}\right)^{T} p_{k}\right)} .
$$

Se $\alpha_{\text {novo }} \geq 0.1 \alpha_{\text {tent }} \ell \alpha_{\text {novo }} \leq 0.9 \alpha_{\text {tent }}$ então faça $\alpha_{\text {novo }} \leftarrow \alpha_{\text {novo }}$. Senão, faça $\alpha_{\text {novo }} \leftarrow$ $0.5 \alpha_{\text {tent }}$.

Faça $\alpha_{\text {tent }} \leftarrow \alpha_{\text {novo }}$ e volte ao Passo 3 . 
Algoritmo 9 Extrapolação

Dados $x_{k}$ viável, $p_{k}$ direção de descida, $\mu \in(0,1]$ tal que $x_{k+1}=x_{k}+\mu p_{k}$ e $N>1$.

Passo 1. Decisão de extrapolar ou não

Se $p_{k}^{T} \nabla \bar{f}\left(x_{k}+p_{k}\right) \geq 0.5 p_{k}^{T} \nabla \bar{f}\left(x_{k}\right)$ então PARE.

Passo 2. Calcule $\mu_{\max }=\max \left\{t \geq 0 \mid\left[x_{k}, x_{k}+t p_{k}\right] \subset \Omega\right\}$.

Passo 3. Cálculo do novo escalar que multiplica $p_{k}$

Se $\mu<\mu_{\max }$ e $N_{\mu}>\mu_{\max }$ então faça $\mu_{\text {tent }} \leftarrow \mu_{\max }$. Senão, faça $\mu_{\text {tent }} \leftarrow N_{\mu}$.

Passo 4. Continua extrapolação enquanto obtém decréscimo simples da função $\mathrm{Se}$

$$
\bar{f}\left(P_{\Omega}\left(x_{k}+\mu p_{k}\right)\right) \leq \bar{f}\left(P_{\Omega}\left(x_{k}+\mu_{\text {tent }} p_{k}\right)\right)
$$

então PARE e devolva $P_{\Omega}\left(x_{k}+\mu p_{k}\right)$. Senão, faça $\mu \leftarrow \mu_{\text {tent }}$ e volte para o Passo 3 . 


\section{Superfícies e parametrizações}

Neste apêndice vamos fazer uma revisão teórica de conceitos topológicos e geométricos sobre superfícies e parametrizações. Estes conceitos são usados na definição de malhas e na formulação do modelo de programação não-linear usado para otimizá-las.

O material apresentado é baseado nos livros Fitzpatrick (2006); Gallier (2011); do Carmo (1976); Pressley (2010); Bloch (1997); Gray (1994); do Carmo (1971); Guibas \& Stolfi (1985); Frey \& George (2000).

As provas dos teoremas, proposições e lemas enunciados neste apêndice encontram-se no Apêndice C.

\section{B.1 Superfícies}

Seja $\mathbb{E}^{n}$ um espaço Euclidiano afim $n$-dimensional sobre $\mathbb{R}$ e seja $\mathbb{R}^{n}$ um espaço vetorial associado a $\mathbb{E}^{n}$. Lembre-se que $\mathbb{R}^{n}$ é provido de um produto interno, o qual vamos denotar por $\langle$,$\rangle .$

Definição B.1. Uma curva paramétrica diferenciável é uma função suave $\alpha: I \rightarrow \mathbb{E}^{3}$, para um intervalo aberto $I=(a, b)$ de $\mathbb{E}$. Se a derivada $\alpha^{\prime}(p) \neq 0$, para todo ponto $p \in I$, então dizemos que a curva $\alpha$ é regular.

Definição B.2. Seja $f: U \rightarrow W$ uma função contínua e bijetora. Se a inversa $f^{-1}: W \rightarrow$ $U$ é também contínua, então $f$ é dito um homeomorfismo e os conjuntos $U$ e $W$ são ditos homeomórficos. 
Definição B.3. Um subconjunto $S$ de $\mathbb{E}^{3}$ é uma superfície se, para todo ponto $p \in S$, existem um conjunto aberto $U$ em $\mathbb{E}^{2}$, um conjunto aberto $W$ em $\mathbb{E}^{3}$, que contém $p$, e um mapa $X: U \rightarrow \mathbb{E}^{3}$ tal que o seguinte vale:

1. $X(U)=S \cap W$,

2. $X: U \rightarrow(S \cap W)$ é um homeomorfismo, $e$

3. o diferencial de $X$ em $q,(d X)_{q}: \mathbb{R}^{2} \rightarrow \mathbb{R}^{3}$, é injetor para todo $q \in U$.

Podemos escrever $X$ em termos de suas componentes como $X(u, v)=(x(u, v)$, $y(u, v), z(u, v))$. Então, dizer que $X$ é suave é equivalente a dizer que $x, y$ e $z$ são suaves (isto é, as componentes da função $x, y$ e $z$ tem derivadas contínuas de ordem $k$, para todo $k$ inteiro positivo). Se $X$ é suave e satisfaz a condição 1 , então $X: U \rightarrow(S \cap W)$ é contínuo e sobrejetor. A condição 2 força $X$ a ser injetor, impedindo que $X(U)$ tenha auto-intersecções. Se o mapa $(d X)_{q}$ é injetor, então os vetores

$$
X_{u}(q)=\frac{\partial X}{\partial u}(q) \text { e } X_{v}(q)=\frac{\partial X}{\partial v}(q)
$$

são linearmente independentes. Assim, somos capazes de definir um plano tangente a $S$ em $q$. O mapa $X$ na Definição B.3 é chamado parametrização de $S$, enquanto as variáveis $u$ e $v$ são chamadas coordenadas locais de $S$.

Da Definição B.3, vemos que a superfície é a união de imagens de uma coleção de parametrizações. Então, se um ponto $p$ pertence ao raio de alcance de duas diferentes parametrizações, teremos dois sistemas de coordenadas junto a $p$. Mais especificamente, sejam $X_{i}: U_{i} \rightarrow X_{i}(U) \subseteq S$, para $i=1,2$, duas parametrizações de $S$ tal que $\Omega=X_{1}\left(U_{1}\right) \cap X_{2}\left(U_{2}\right)$ é não vazio. Então o mapa

$$
h=X_{2}^{-1} \circ X_{1}: X_{1}^{-1}(\Omega) \rightarrow X_{2}^{-1}(\Omega)
$$

é um homeomorfismo tomando coordenadas em $U_{1}$ com respeito a $X_{1}$ e coordenadas em $U_{2}$ com respeito a $X_{2}$. O mapa $h$ é dito mudança de parâmetros ou mudança de coordenadas.

Definição B.4. Duas superfícies regulares $S_{1}$ e $S_{2}$ são difeomórficas se existe uma aplicação diferenciável $f: S_{1} \rightarrow S_{2}$ com inversa $f_{1}^{-1}: S_{2} \rightarrow S_{1}$ diferenciável, ou seja, $f$ é um difeomorfismo de $S_{1}$ sobre $S_{2}$.

A noção de difeomorfismo desempenha para superfícies regulares o mesmo papel que a noção de isomorfismo desempenha para espaços vetoriais.

Podemos verificar que o mapa $h$, definido acima, é um difeomorfismo. Para provar essa afirmação, usaremos o Teorema da Função Inversa. 
Teorema B.1. (Teorema da Função Inversa) Seja $f: U \subset \mathbb{E}^{n} \rightarrow \mathbb{E}^{n}$ uma função diferenciável tal que $(d f)_{q}: \mathbb{R}^{n} \rightarrow \mathbb{R}^{n}$ é bijetora em um ponto $q \in U$. Então, existem conjuntos abertos $V$ e $W$, com $V \subset U$ e $W \subset f(V)$, de modo que a restrição $f_{\mid V}: V \rightarrow W$, de $f$ em $V$, é um difeomorfismo de $V$ em $W$.

A prova do Teorema B.1 pode ser encontrada em Fitzpatrick (2006).

Lema B.1. Sejam $S$ uma superfície e $X: U \rightarrow X(U) \subseteq S$ uma parametrização na qual a imagem contém o ponto $p$. Então, existe um subconjunto aberto $V$ de $U$ contendo o ponto $q e$ uma projeção ortogonal $\pi: \mathbb{E}^{3} \rightarrow \mathbb{R}^{2}$ sobre alguma das três coordenadas planas de $\mathbb{R}^{3}$ tal que $W=(\pi \circ X)(V)$ é aberto em $\mathbb{E}^{2}$ e tal que $\pi \circ X: V \rightarrow W$ é um difeomorfismo.

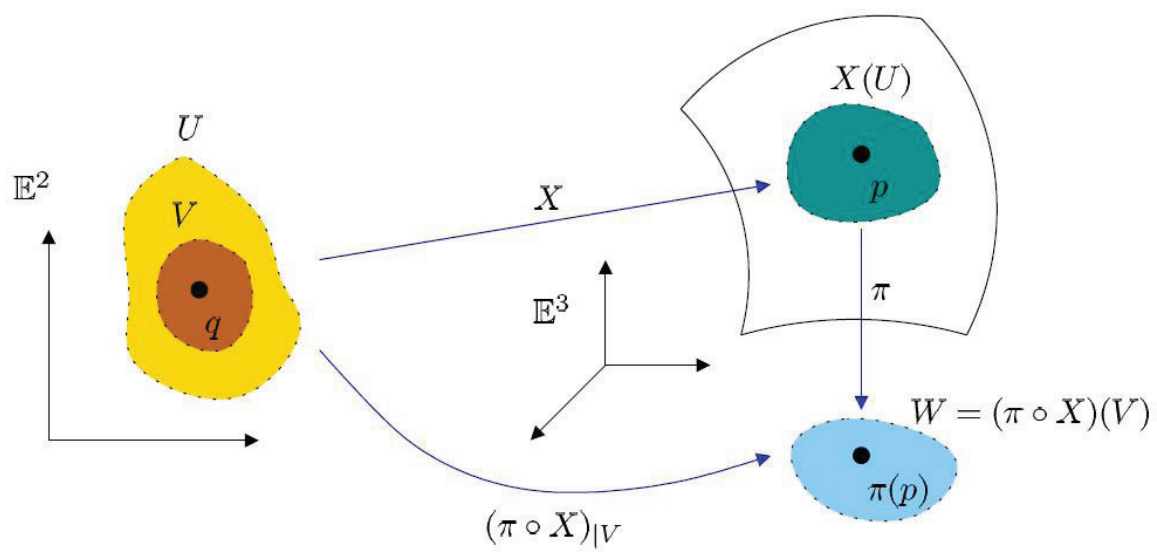

Figura B.1: Ilustração do Lema B.1.

Teorema B.2. Toda mudança de coordenadas é um difeomorfismo.

É importante lembrar que se $X_{1}$ e $X_{2}$ são diferenciáveis, não implica que $h=X_{2}^{-1} \circ$ $X_{1}$ é diferenciável. A razão é que $X_{2}^{-1}$ é definido em $X_{2}(U) \subset S$, e não sabemos o que significa ser "diferenciável" em outro espaço a não ser no $\mathbb{R}^{n}$. Por outro lado, o mapa $\left(\pi \circ X_{2}\right)^{-1}$ é definido em $U_{2}$ e provamos que $\left(\pi \circ X_{2}\right)^{-1}$ é um difeomorfismo (veja prova do Lema B.1).

\section{B.2 Primeira Forma Fundamental}

Definição B.5. Sejam $S$ uma superfície e p um ponto em $S$. Dizemos que o vetor $v \in \mathbb{R}^{3}$ é tangente a $S$ em p se existe uma parametrização de uma curva diferenciável $\alpha:(-\varepsilon, \varepsilon) \rightarrow S$, com $\varepsilon>0$, tal que $\alpha(0)=p$ e $\alpha^{\prime}(0)=v$. O conjunto que contém os vetores tangentes a $S$ no ponto pé denotado por $T_{p} S$.

Lema B.2. Sejam $S$ uma superfície, $p \in S$ e $X: U \rightarrow X(U) \subseteq S$ uma parametrização de $S$ $\operatorname{com} p \in X(U)$. Então,

$$
T_{p} S=(d X)_{X^{-1}(p)}\left(\mathbb{R}^{2}\right) .
$$




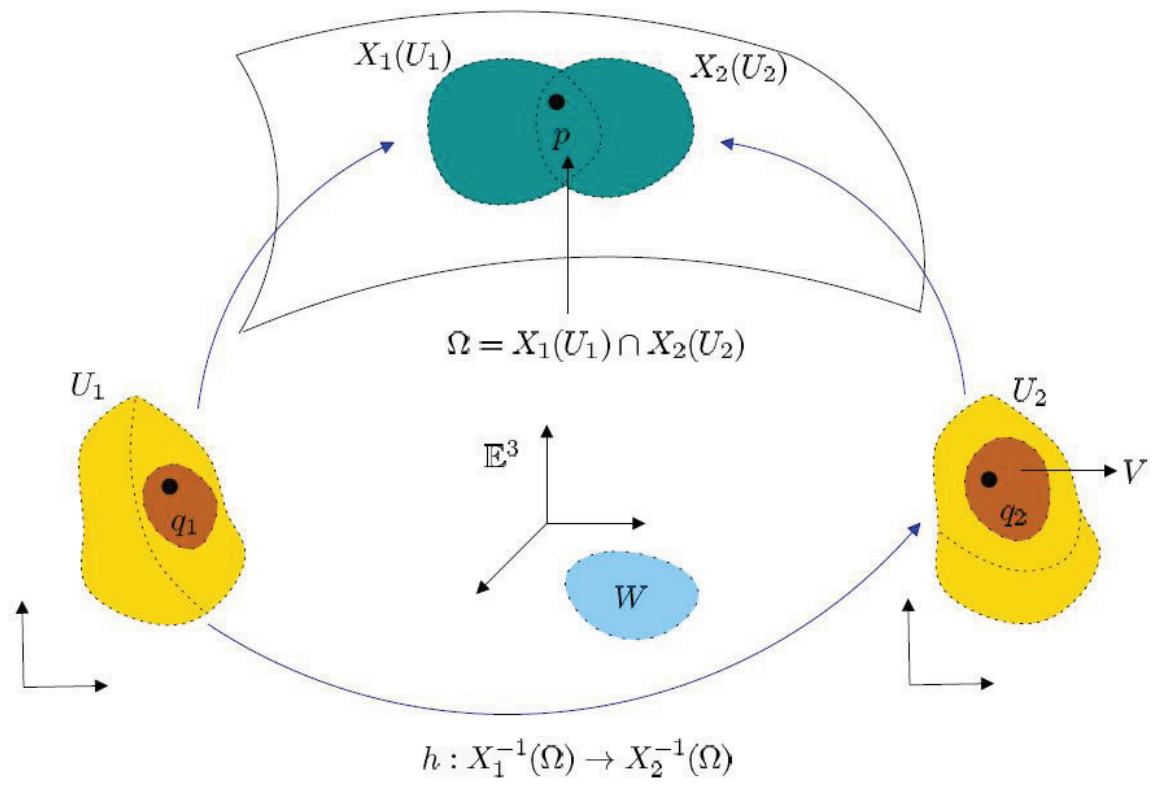

Figura B.2: Ilustração da mudança de coordenadas.

Pelo Lema B.2, o conjunto $T_{p} S$ formado por todos os vetores tangentes à superfície $S$ em um dado ponto $p \in S$ é um espaço linear em $\mathbb{R}^{3}$, o qual chamaremos de espaço tangente à superfície $S$ no ponto $p$. O conjunto de retas tangentes passando por $p$ que tem um vetor tangente em $T_{p} S$ como direção é um plano afim chamado plano tangente afim no ponto $p$. Geometricamente, é um objeto diferente de $T_{p} S$. Se $X: U \rightarrow X(U) \subseteq S$ é uma parametrização de $S$ tal que $p \in X(U)$, então o Lema B.2 nos diz que $(d X)_{q}\left(\mathbb{R}^{2}\right)$ não depende de $X$ e que $\left\{X_{u}(q), X_{v}(q)\right\}$, com $q \in X^{-1}(p)$, é uma base do espaço $T_{p} S$.

Escolhemos o produto interno para $\mathbb{R}^{3}$ induzindo um produto interno para cada espaço tangente $T_{p} S$ de uma superfície $S$ em um ponto $p$. Esse produto interno é denotado por $\langle,\rangle_{p}$ e é definido como segue: se $v, w \in T_{p} S \subset \mathbb{R}^{3}$, então $\langle v, w\rangle_{p}$ é igual ao produto interno de $v$ e $w$ considerando os vetores em $\mathbb{R}^{3}$.

Definição B.6. Sejam $S$ uma superfície, $p \in S$ e $X: U \rightarrow X(U) \subseteq S$ uma parametrização de $S$, com $p \in X(U)$. Então, o mapa

$$
I_{p}: T_{p} S \rightarrow \mathbb{R}
$$

dado por

$$
I_{p}(w)=\langle w, w\rangle_{p}=\|w\|_{p}^{2},
$$

para todo $w \in T_{p} S$, é a Primeira Forma Fundamental para $S$ de $p$.

A Primeira Forma Fundamental da superfície nos permite estabelecer uma propriedade métrica local na superfície, tais como o comprimento dos arcos das curvas na superfície e dos ângulos definidos pelos vetores tangentes, sem nenhuma consideração do espaço ambiente, 
$\mathbb{R}^{3}$, onde está a superfície. Podemos expressar a Primeira Forma Fundamental usando a base $\left\{X_{u}, X_{v}\right\}$ associada à parametrização $X(u, v)$ e cobrindo um ponto $p$ em $S$. Visto que um vetor $w \in T_{p} S$ é o vetor tangente de uma curva paramétrica diferenciável $\alpha(t)=X(u(t), v(t))$, tal que $t \in(-\varepsilon, \varepsilon)$, para algum $\varepsilon>0$, e tal que $p=\alpha(0)=X\left(u_{0}, v_{0}\right)$, temos

$$
\begin{aligned}
I_{p}\left(\alpha^{\prime}(0)\right) & =\left\langle\alpha^{\prime}(0), \alpha^{\prime}(0)\right\rangle_{p} \\
& =\left\langle X_{u} u^{\prime}+X_{v} v^{\prime}, X_{u} u^{\prime}+X_{v} v^{\prime}\right\rangle_{p} \\
& =\left\langle X_{u}, X_{v}\right\rangle_{p}\left(u^{\prime}\right)^{2}+2\left\langle X_{u}, X_{v}\right\rangle_{p} u^{\prime} v^{\prime}+\left\langle X_{u}, X_{v}\right\rangle_{p}\left(v^{\prime}\right)^{2} \\
& =E\left(u^{\prime}\right)^{2}+2 F u^{\prime} v^{\prime}+G\left(v^{\prime}\right)^{2},
\end{aligned}
$$

onde os valores da função são computados em $t=0$ e $E\left(u_{0}, v_{0}\right)=\left\langle X_{u}, X_{u}\right\rangle_{p}, F\left(u_{0}, v_{0}\right)=$ $\left\langle X_{u}, X_{v}\right\rangle_{p}$ e $G\left(v_{0}, v_{0}\right)=\left\langle X_{v}, X_{v}\right\rangle_{p}$ são os coeficientes da Primeira Forma Fundamental na base $\left\{X_{u}, X_{v}\right\}$ de $T_{p} S$. Por variar $p$ no sistema de coordenadas local definido por $X(u, v)$, temos que as funções $E(u, v), F(u, v)$ e $G(u, v)$ são diferenciáveis com respeito às coordenadas $u \mathrm{e}$ $v$.

A Primeira Forma Fundamental $I_{p}$ é frequentemente escrita na forma matricial como

$$
I_{p}(x, y)=\left(\begin{array}{ll}
x & y
\end{array}\right)\left(\begin{array}{ll}
E & F \\
F & G
\end{array}\right)\left(\begin{array}{l}
x \\
y
\end{array}\right) \text {, }
$$

onde supomos que $x$ e $y$ são as coordenadas de um vetor $w \in T_{p} S$ com respeito à base $\left\{X_{u}, X_{v}\right\}$ de $T_{p} S$, isto é, $w=x \cdot X_{u}+y \cdot X_{v}$. Isso significa que $I_{p}(x, y)=E x^{2}+2 F x y+G y^{2}$ é a forma quadrática definida positiva. A razão para isso é que $\left(E G-F^{2}\right)^{\frac{1}{2}}$ é igual a $\left\|X_{u} \times X_{v}\right\|$. Daí a proposição a seguir.

Proposição B.1. Sejam $S$ uma superfície, $p \in \S, E, F, G$ são os coeficientes da Primeira Forma Fundamental na base na base $\left\{X_{u}, X_{v}\right\}$ de $T_{p} S$. Então

$$
\left\|X_{u} \times X_{v}\right\|=\left(E G-F^{2}\right)^{\frac{1}{2}}
$$

Como $X_{u} \times X_{v} \neq 0$, temos que $\left\|X_{u} \times X_{v}\right\|>0$, ou seja, $E>0, G>0$, e $E G-F^{2}>0$. Então,

$$
\left(\begin{array}{ll}
E & F \\
F & G
\end{array}\right)
$$

é uma matriz real, simétrica, definida positiva e, deste modo, seus autovalores são números reais positivos. Note que os coeficientes $E, F$ e $G$ e os coeficientes do vetor $w$ em (B.4) depende da escolha de uma parametrização $X$ de $S$, mas a Primeira Forma Fundamental depende somente de $S$ e $p$. 


\section{B.2.1 Comprimento}

Com a Primeira Forma Fundamental podemos calcular o comprimento das curvas regulares na superfície. De fato, se

$$
\alpha:(a, b) \rightarrow S
$$

é uma curva regular em $S$ então o comprimento $s(t)$ de $\alpha$ entre $\alpha\left(t_{0}\right)$ e $\alpha(t)$ ao longo de $\alpha$ é definido por

$$
s(t)=\int_{t_{0}}^{t}\left\|\alpha^{\prime}(r)\right\| d r=\int_{t_{0}}^{t} \sqrt{\left\langle\alpha^{\prime}(r), \alpha^{\prime}(r)\right\rangle_{\alpha(r)}} d r=\int_{t_{0}}^{t}\left\|\sqrt{I_{\alpha(r)}\left(\alpha^{\prime}(r)\right)}\right\| d r .
$$

Usando variáveis locais, temos

$$
s(t)=\int_{t_{0}}^{t} \sqrt{E(u(r), v(r))\left(\frac{d u}{d r}\right)^{2}+2 F(u(r), v(r)) \frac{d u}{d r} \frac{d v}{d r}+G(u(r), v(r))\left(\frac{d v}{d r}\right)^{2}} d r .
$$

Isto é normalmente abreviado pela equação

$$
d s^{2}=E d u^{2}+2 F d u d v+G d v^{2}
$$

onde ds é chamado elemento do comprimento do arco ou elemento do arco em $S$. expressão para o comprimento do arco de uma curva sobre uma superfície, dada localmente pelo elemento do arco, é algo que deve depender apenas da curva e não da escolha de uma parametrização de $S$. Isto é de fato o caso da proposição B.2

Proposição B.2. Sejam E, F, G são os coeficientes da Primeira Forma Fundamental na base na base $\left\{X_{u}, X_{v}\right\}$ de $T_{p} S$ e ds é chamado elemento do comprimento do arco. Então, a expressão $d s^{2}=E d u^{2}+2 F d u d v+G d v^{2}$ não é alterada por uma reparametrização.

\section{B.2.2 Ângulo}

O ângulo $\theta$ entre duas curvas regulares $\alpha, \beta:(-\varepsilon, \varepsilon) \rightarrow S$, com intersecção em $t=t_{0}$ e $s=s_{0}$, é dado por

$$
\theta=\arccos \left(\frac{\left\langle\alpha^{\prime}\left(t_{0}\right), \beta^{\prime}\left(s_{0}\right)\right\rangle_{p}}{\left\|\alpha^{\prime}\left(t_{0}\right)\right\|_{p} \cdot\left\|\beta^{\prime}\left(s_{0}\right)\right\|_{p}}\right)
$$

onde $p=\alpha\left(t_{0}\right)=\beta\left(s_{0}\right)$. Em particular, o ângulo $\phi$ entre a $u$-curva $\gamma(t)=X\left(u_{0}, t\right)$ e a $v$-curva $\rho(s)=X\left(s, v_{0}\right)$ de $X(u, v)$, passando por $p$, onde $u_{0}=u\left(t_{0}\right)$ e $v_{0}=v\left(s_{0}\right)$, é dado por

$$
\phi=\arccos \left(\frac{\left\langle X_{u}, X_{v}\right\rangle_{p}}{\left\|X_{u}\right\|_{p} \cdot\left\|X_{v}\right\|_{p}}\right)=\arccos \left(\frac{F}{\sqrt{E G}}\right) .
$$


Assim, a $u$-curva e a $v$-curva de $X(u, v)$ são orthogonais se, e somente se, $F(u, v)=$ 0 no domínio $U$ de $X$.

Usualmente, é útil ter a expressão para $\theta$ em termos da parametrização. De fato, sejam

$$
\alpha^{\prime}(t)=X_{u} \frac{d u}{d t}+X_{v} \frac{d v}{d t} \quad \text { e } \quad \beta^{\prime}(s)=X_{u} \frac{d u}{d s}+X_{v} \frac{d v}{d s} .
$$

Temos que

$$
\theta=\arccos \left(\frac{E \frac{d u}{d t} \frac{d v}{d s}+F\left(\frac{d u}{d t} \frac{d u}{d s}+\frac{d u}{d s} \frac{d v}{d t}\right)+G \frac{d v}{d t} \frac{d v}{d s}}{\left(E\left(\frac{d u}{d t}\right)^{2}+2 F \frac{d u}{d t} \frac{d v}{d t}+G\left(\frac{d v}{d t}\right)^{2}\right)^{\frac{1}{2}}\left(E\left(\frac{d u}{d s}\right)^{2}+2 F \frac{d u}{d s} \frac{d v}{d s}+G\left(\frac{d v}{d s}\right)^{2}\right)^{\frac{1}{2}}}\right)
$$

Como esperado, a expressão B.10 também é invariante quando ocorre uma mudança de coordenadas.

Proposição B.3. Sejam E, F, G são os coeficientes da Primeira Forma Fundamental na base na base $\left\{X_{u}, X_{v}\right\}$ de $T_{p} S$ e ds é chamado elemento do comprimento do arco. Então, a expressão (B.10) não é alterada por uma reparametrização.

\section{B.2.3 Área}

Uma região $D$ do plano é um subconjunto limitado e fechado de $\mathbb{E}^{2}$ cujo interior é homeomórfico a um disco aberto em $\mathbb{E}^{2}$ e cujo limite (isto é, o subconjunto de $\mathbb{E}^{2}$ homeomórfico a uma circunferência) consiste na união de imagens de curvas regulares finitas. Se $X: U \rightarrow$ $X(U) \subseteq S$ é uma parametrização de uma superfície $S$ e $D \subset U$ é uma região de $\mathbb{E}^{2}$, então dizemos que $X(D)$ é uma região de superfície.

A Definição B.1 é um resutado padrão no cálculo de várias variáveis.

Definição B.1. Sejam $S$ uma superfície, $X: U \rightarrow X(U) \subseteq S$ uma parametrização definida em um subconjunto aberto $U$ de $\mathbb{E}^{2}$, e $D$ uma região de $\mathbb{E}^{2}$ com $D \subset U$. Então, a área $A_{X}(D)$ de uma região de superfície $X(D) \subseteq S$ é

$$
\int_{D}\left\|X_{u} \times X_{v}\right\| d u d v
$$

A exigência de que $D$ seja uma região do plano garante que a função $\left\|X_{u} \times X_{v}\right\|$ da Definição B.1 é integrável e tem um valor finito, que é a área de $X(D)$. A razão para isso é que, por definição, $D$ é um subconjunto compacto de $\mathbb{E}^{2}$ e $X_{u}$ e $X_{v}$ são contínuos. Então, a função $\left\|X_{u} \times X_{v}\right\|$ é também contínua. Deste modo, pelo Teorema do Valor Extremo (veja Fitzpatrick (2006)), $\left\|X_{u} \times X_{v}\right\|$ é limitado em $D$.

Algumas vezes vamos denotar $\left(E G-F^{2}\right)^{\frac{1}{2}} d u d v$ por $d A_{X}$. É possível mostrar que a Definição B.1 não depende da escolha da parametrização $X$, isto é, a área $A_{X}(D)$ não muda se 
reparametrizarmos $S$. Isso certamente não é óbvio, já que $E, F$ e $G$ mudam com a reparametrização.

Proposição B.4. A área de uma região de uma superfície não é mudada por uma reparametrização.

\section{B.3 Mapas conformais}

Definição B.7. Seja $S$ uma superfície. Então, a função $f: S \rightarrow \mathbb{E}^{n}$ é diferenciável em um ponto $p$ de $S$ se existe uma parametrização $X: U \rightarrow X(U) \subseteq S$ tal que $p \in X(U)$ e $f \circ X$ é diferenciável em $X^{-1}(p)$. Assim, usualmente dizemos que $f: S \rightarrow \mathbb{E}^{n}$ é diferenciável se $f$ é diferenciável em todo ponto $p$ de $S$.

A Figura B.3 ilustra a Definição B.7.

Note que, se $f \circ X$ é diferenciável em $X^{-1}(p)$, a função $f \circ Y$ deve ser diferenciável em $Y^{-1}(p)$, para toda parametrização $Y: W \rightarrow Y(W) \subseteq S$, com $w \subset \mathbb{R}^{2}$ e $p \in Y(W)$. De fato, o Teorema B.2 nos diz que $X^{-1} \circ Y$ é diferenciável em $Y^{-1}$. Como $f \circ X$ é, por hipótese, diferenciável em $X^{-1}(p)$, a regra da cadeia implica que $f \circ Y=(f \circ X) \circ\left(X^{-1} \circ Y\right)$ é diferenciável em $Y^{-1}(p)$.

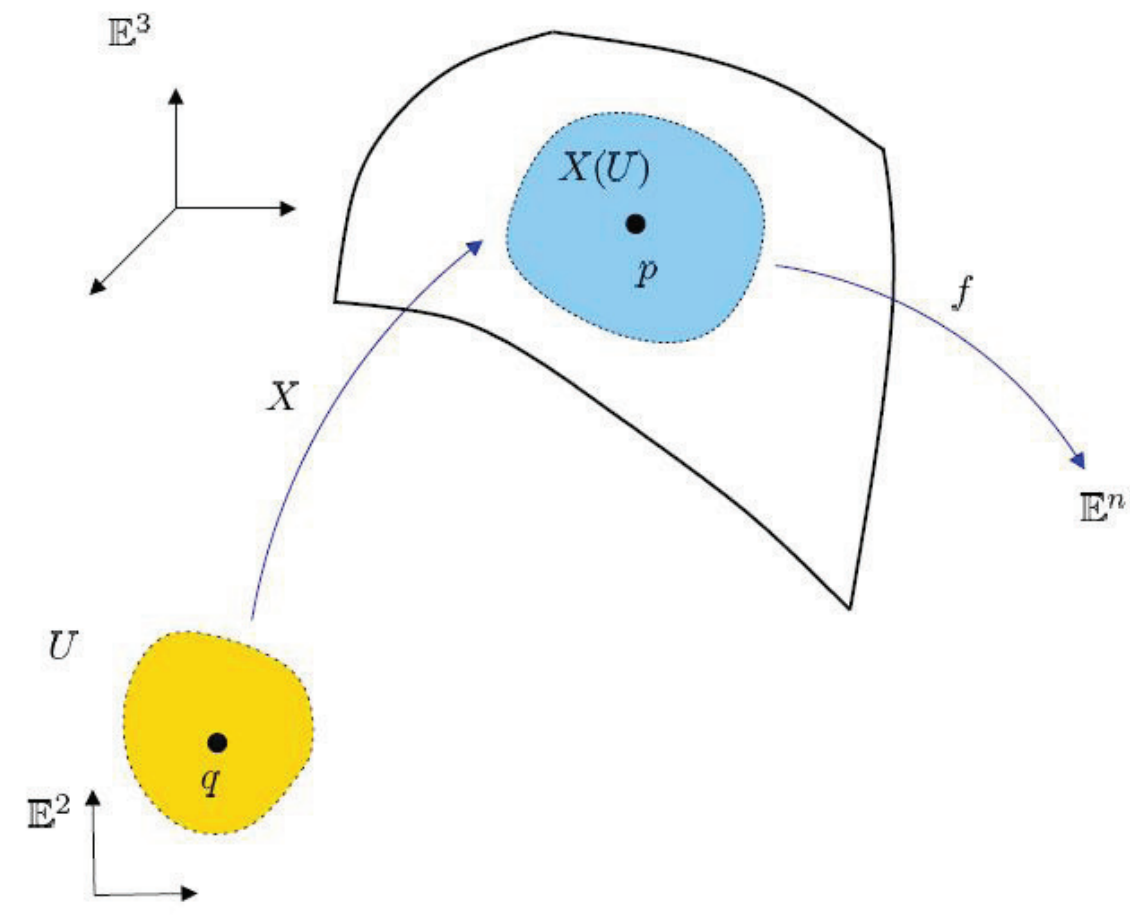

Figura B.3: Função $f$ é diferenciável em $p$ se $f \circ X$ for diferenciável em $p$. 
Definição B.8. Seja $S$ uma superfície. Então, a função $f: \mathbb{E}^{n} \rightarrow S$ é diferenciável se a função composta $i \circ f: \mathbb{E}^{n} \rightarrow \mathbb{E}^{3}$ é diferenciável, quando $i: S \rightarrow \mathbb{E}^{3}$ é a inclusão $i(p)=$ p, para todo $p \in S$.

Definição B.9. Sejam $S_{1}$ e $S_{2}$ duas superfícies, e seja $f: S_{1} \rightarrow S_{2}$. Então, dizemos que $f$ é diferenciável em um ponto $p$ em $S_{1}$ se existem duas parametrizações, $X: U \rightarrow X(U) \subseteq S_{1}$ e $Y: W \rightarrow Y(W) \subseteq S_{2}$, tais que $p \in X(U),(f \circ X)(U) \subseteq Y(W)$ e a função $\varphi_{X Y}: U \rightarrow W$, onde $\varphi_{X Y}=\left(Y^{-1} \circ f \circ X\right)$, é diferenciável em $X^{-1}(p)$. Usualmente, dizemos que $f: S_{1} \rightarrow S_{2}$ é diferenciável se f é diferenciável em qualquer ponto $\mathrm{p}$ em $S_{1}$.

Suponha que a função $\varphi_{X Y}=\left(Y^{-1} \circ f \circ X\right)$ da Definição B.9 seja diferenciável em um ponto $q=X^{-1}(p)$. Sejam $\widetilde{X}: \widetilde{U} \rightarrow X(\widetilde{U}) \subseteq S_{1}$ e $\widetilde{Y}: \widetilde{W} \rightarrow Y(\widetilde{W}) \subseteq S_{2}$ duas parametrizações tais que $p \in X(\widetilde{U})$ e $(f \circ \tilde{X})(\widetilde{U}) \subseteq Y(\widetilde{W})$. Então, a função $\varphi_{\widetilde{X} \widetilde{Y}}=\left(\widetilde{Y}^{-1} \circ\right.$ $f \circ \tilde{X})$ é diferenciável em $\widetilde{X}^{-1}(p)$. De fato,

$$
\begin{aligned}
\varphi_{\tilde{X} \tilde{Y}} & =\left(\tilde{Y}^{-1} \circ f \circ \widetilde{X}\right) \\
& =\widetilde{Y}^{-1} \circ\left(Y \circ Y^{-1}\right) \circ f \circ\left(X \circ X^{-1}\right) \circ \widetilde{X} \\
& =\left(\widetilde{Y}^{-1} \circ Y\right) \circ\left(Y^{-1} \circ f \circ X\right) \circ\left(X^{-1} \circ \widetilde{X}\right) .
\end{aligned}
$$

Do Teorema B.2, temos que $\left(\widetilde{Y}^{-1} \circ Y\right)$ e $\left(X^{-1} \circ \widetilde{X}\right)$ são diferenciáveis em $\left(Y^{-1} \circ f\right)(p)$ e $\widetilde{X}^{-1}(p)$, respectivamente. Por hipótese, a função $\varphi_{X Y}=\left(Y^{-1} \circ f \circ X\right)$ é diferenciável em $X^{-1}(p)$. Então, pela regra da cadeia, a função $\varphi_{\tilde{X} \widetilde{Y}}=\left(\widetilde{Y}^{-1} \circ f \circ \widetilde{X}\right)$ deve ser diferenciável em $\widetilde{X}^{-1}(p)$. Isso significa que, se $f$ é diferenciável em $p$ com respeito a $\varphi_{X Y}$, também será diferenciável em $p$ com respeito a qualquer função $\varphi_{\tilde{X} \widetilde{Y}}$ tal que $\widetilde{X}$ e $\widetilde{Y}$ satisfaçam as condições $p \in X(\widetilde{U})$ e $(f \circ \widetilde{X})(\widetilde{U}) \subseteq Y(\widetilde{W})$ na Definição B.9.

A Figura B.4 ilustra a Definição B.9.

Definição B.10. Sejam $S_{1}$ e $S_{2}$ duas superfícies, e seja $f: S_{1} \rightarrow S_{2}$ um difeomorfismo. Então, dizemos que $f$ é conformal se, e somente se, para todo ponto p em $S_{1}$, o ângulo definido por alguma das duas curvas regulares $\alpha$ e $\widetilde{\alpha}$ em $S_{1}$ que se intersectam em $p$ é igual ao ângulo definido em $f(p)$ pelas curvas $\beta=f \circ \alpha$ e $\widetilde{\beta}=f \circ \widetilde{\alpha}$ em $S_{2}$.

Geometricamente o significado da Definição B.10 é que ângulos são preservados por mapas conformais. O fato de $f$ ser um difeomorfismo garante que $\beta=f \circ \alpha$ e $\widetilde{\beta}=f \circ \widetilde{\alpha}$ são curvas regulares. Então, o ângulo entre as curvas $\beta$ e $\widetilde{\beta}$ que se intersectam em $f(p)$ é bem definido. É imediatamente verificado que a composição de mapas conformais e as inversas de mapas conformais são também mapas conformais.

Como veremos a seguir, estamos interessados em um caso especial onde a função $f$ é uma parametrização $X: U \rightarrow X(U) \subseteq S$. Mais especificamente, podemos considerar $U$ como um subconjunto aberto de um plano em $\mathbb{E}^{3}$, parametrizado pelas coordenadas $u$ e $v$ no modo usual. Então, o subconjunto $U$ torna-se a superfície $S_{1}$ na Definição B.10, enquanto $X(U)$ 


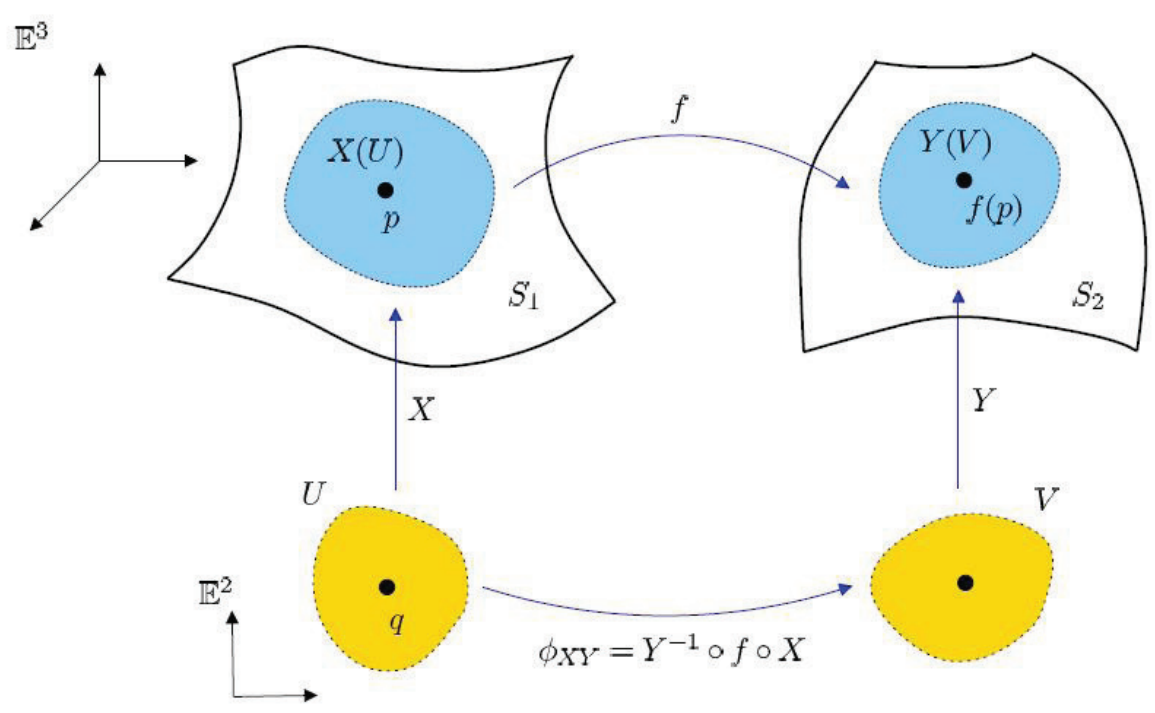

Figura B.4: Função $f$ é diferenciável em $p$ se $\phi_{X Y}$ for diferenciável em $q=X^{-1}(p)$.

torna-se a superfície $S_{2}$. Dizemos que $X$ é uma parametrização conformal se, e somente se, a função $X$, que é um mapa entre superfícies, é conformal.

Teorema B.3. Sejam $S_{1}$ e $S_{2}$ duas superfícies. Um difeomorfismo $f: S_{1} \rightarrow S_{2}$ é conformal se, e somente se, existe uma função $\lambda: S \rightarrow \mathbb{R}$ tal que, para cada $p \in S_{1}$ e para quaisquer dois vetores $v, w \in T_{p} S_{1}$, temos

$$
\left\langle(d f)_{p}(v),(d f)_{p}(w)\right\rangle_{f(p)}=\lambda(p) \cdot\langle v, w\rangle_{p}
$$

\section{B.4 Mapas equiareais}

Definição B.11. Sejam $S_{1}$ e $S_{2}$ duas superfícies e $f: S_{1} \rightarrow S_{2}$ um difeomorfismo. Dizemos que $f$ é equiareal (ou preserva área) se ela toma alguma região em $S_{1}$ e leva a alguma região de mesma área em $S_{2}$.

Vamos relatar uma importante característica de mapas equiareais:

Teorema B.4. Sejam $S_{1}$ e $S_{2}$ duas superfícies. Um difeomorfismo $f: S_{1} \rightarrow S_{2}$ é equiareal se, e somente se, para qualquer parametrização $X: U \rightarrow X(U) \subseteq S_{1}$ e para cada $p \in X(U)$, os coeficientes, $E_{1}, F_{1}, G_{1}$ e $E_{2}, F_{2}, G_{2}$ da Primeira Forma Fundamental de $S_{1}$ e $S_{2}$ em p e $f(p)$ com respeito a $X$ e $f \circ X$, respectivamente, satisfaz

$$
E_{1} \circ G_{1}-F_{1}^{2}=E_{2} \circ G_{2}-F_{2}^{2} .
$$




\section{B.5 Mapas isométricos}

Definição B.12. Sejam $S_{1}$ e $S_{2}$ duas superfícies e $f: S_{1} \rightarrow S_{2}$ um mapa suave de $S_{1}$ para $S_{2}$. Dizemos que f é uma isometria local se ela toma alguma curva em $S_{1}$ e leva a alguma curva de mesmo comprimento em $S_{2}$. Se uma isometria local $f: S_{1} \rightarrow S_{2}$ existe, então $S_{1}$ e $S_{2}$ são ditos localmente isométricos. Se uma isometria local $f: S_{1} \rightarrow S_{2}$ é um difeomorfismo, então f é chamada isometria e $S_{1}$ e $S_{2}$ são ditos isométricos.

É facil verificar que toda composição de isometrias é uma isometria, e que a inversa de uma isometria é uma isometria. O teorema a seguir dá uma condição necessária e suficiente para a isometria local.

Teorema B.5. Sejam $S_{1}$ e $S_{2}$ duas superfícies. Um mapa suave $f: S_{1} \rightarrow S_{2}$ é uma isometria local se, e somente se,

$$
\langle v, w\rangle_{p}=\left\langle(d f)_{p}(v),(d f)_{p}(w)\right\rangle_{f(p)},
$$

para todo ponto p em $S_{1}$ e para cada dois vetores $v$ e $w$ em $T_{p} S_{1}$.

O Teorema B.5 implica que o mapa $(d f)_{p}: T_{p} S_{1} \rightarrow T_{f(p)} S_{2}$ é uma isometria linear (veja Gallier (2011)), isto é, preserva comprimento. De fato, para quaisquer dois vetores $v$ e $w$ em $T_{p} S_{1}$, temos $\left\langle(d f)_{p}(v),(d f)_{p}(w)\right\rangle_{f(p)}=\langle v, w\rangle_{p}$. Além disso, o mapa $(d f)_{p}$ deve ser invertível. Caso contrário, haveria um vetor tangente não nulo, $v$ em $T_{p} S_{1}$ tal que $(d f)_{p}(v)=$ 0 . Mas, como $f$ é uma isometria local, temos que $0 \neq\langle v, v\rangle_{p}=\left\langle(d f)_{p}(v),(d f)_{p}(v)\right\rangle_{f(p)}=$ $\langle 0,0\rangle_{f(p)}=0$, o que é uma contradição. Então, o mapa $(d f)_{p}$ é invertível. O fato que $(d f)_{p}$ é invertível implica que a função $f$ é, localmente, um difeomorfismo.

O colorário seguinte expressa o Teorema B.5 em termos de parametrizações.

Corolário B.1. Sejam $S_{1}$ e $S_{2}$ duas superfícies. Então, um mapa suave $f: S_{1} \rightarrow S_{2}$ é uma isometria local se, e somente se, para qualquer parametrização $X: U \rightarrow X(U) \subseteq S_{1}$ e para todo ponto $p \in X(U)$, a Primeira Forma Fundamental de $S_{1}$ e $S_{2}$ em p e $f(p)$ com respeito a $X$ e $f \circ X$, respectivamente, são o mesmo.

\section{B.6 Distorções métricas}

Seja $S$ uma superfície e seja $X: U \rightarrow X(U) \subseteq S$ uma parametrização conformal. Então, pelo Teorema B.3, temos que

$$
\left\langle(d X)_{q}(v),(d X)_{q}(v)\right\rangle_{X(q)}=\lambda(q) \cdot\langle v, v\rangle_{q}
$$


para todo ponto $q \in U$ e para qualquer vetor $v$ em $T_{q} U$. Se tomarmos $X(u, v)=(x(u, v), y(u, v)$, $z(u, v))$, então

$$
J_{X(q)}=\left(\begin{array}{ll}
\frac{\partial x}{\partial u}(q) & \frac{\partial x}{\partial v}(q) \\
\frac{\partial y}{\partial u}(q) & \frac{\partial y}{\partial v}(q) \\
\frac{\partial z}{\partial u}(q) & \frac{\partial z}{\partial v}(q)
\end{array}\right)
$$

é a matriz Jacobiana de $(d X)_{q}$. Logo,

$$
\begin{aligned}
(d X)_{q}(v) & =J_{X(q)} \cdot v\left(\frac{\partial x}{\partial u}(q) \cdot v_{x}+\frac{\partial x}{\partial v}(q) \cdot v_{y}, \frac{\partial y}{\partial u}(q) \cdot v_{x}\right. \\
& \left.+\frac{\partial y}{\partial v}(q) \cdot v_{y}, \frac{\partial z}{\partial u}(q) \cdot v_{x}+\frac{\partial z}{\partial v}(q) \cdot v_{y}\right)
\end{aligned}
$$

e

$$
\begin{aligned}
\left\langle(d X)_{q}(v),(d X)_{q}(v)\right\rangle_{X(q)} & =\left\langle X_{u}, X_{u}\right\rangle_{X(q)} \cdot v_{x}^{2}+2 \cdot\left\langle X_{u}, X_{v}\right\rangle_{X(q)} \cdot v_{x} \cdot v_{y} \\
& +\left\langle X_{v}, X_{v}\right\rangle_{X(q)} \cdot v_{y}^{2} \\
& =E \cdot v_{x}^{2}+2 \cdot F \cdot v_{x} \cdot v_{y}+G \cdot v_{y}^{2} \\
& =I_{X(q)}(v)
\end{aligned}
$$

onde $v_{x}$ e $v_{y}$ são as coordenadas de $v$. Deste modo, $I_{X(q)}(v)=\lambda(q) \cdot\langle v, v\rangle_{q}$. Mas, $\langle v, v\rangle_{q}=$ $I_{q}(v)$ é a Primeira Forma Fundamental da superfície U (relembre de X como um mapa para uma superfície de outra superfície) e parametrizada pelas coordenadas $u$ e $v$ de modo usual. Em particular, se escolhemos dois vetores linearmente independentes, $w_{1}$ e $w_{2}$ em $\mathbb{R}^{3}$, podemos expressar todo ponto de $U$ como $p+u \cdot w_{1}+v \cdot w_{2}$, onde $p$ é qualquer ponto em $U$. Com respeito a esta parametrização da superfície $U$, temos

$$
I_{q}(v)=\left(\begin{array}{ll}
v_{x} & v_{y}
\end{array}\right)\left(\begin{array}{ll}
1 & 0 \\
0 & 1
\end{array}\right)\left(\begin{array}{l}
v_{x} \\
v_{y}
\end{array}\right),
$$

o que implica que

$$
I_{X(q)}(v)=\left(\begin{array}{ll}
v_{x} & v_{y}
\end{array}\right)\left(\begin{array}{cc}
\lambda(q) & 0 \\
0 & \lambda(q)
\end{array}\right)\left(\begin{array}{l}
v_{x} \\
v_{y}
\end{array}\right)
$$

Em outras palavras, as derivadas das $u$-curvas e $v$-curvas passando por $X(q)$ são ortogonais e tem a mesma magnitude, que é consistente com o fato de que $X$ é uma parametrização conformal. Isso é equivalente a dizer que os autovalores da matriz associada de $I_{X(q)}$ são ambos iguais para $\lambda(q)$. Em geral, se $X$ é alguma parametrização de $S$, e se $\lambda_{\max }$ e $\lambda_{\min }$ são os autovalores máximo e mínimo de $I_{X(q)}$, então a conformidade de $X$ pode ser expressada por

$$
\frac{\lambda_{\max }}{\lambda_{\min }}
$$

Como $I_{X(q)}$ é definida positiva, temos $\lambda_{\max } \geq \lambda_{\min }>0$. Então, um é o menor valor da razão. 
Se $X$ é um mapa equiareal, então o Teorema B.4 nos diz que os coeficientes $E_{1}, F_{1}, G_{1}$ e $E_{2}, F_{2}, G_{2}$, de $I_{q}$ e $I_{X(q)}$ devem satisfazer $E 1 \cdot G_{1}-F_{1}^{2}=E 2 \cdot G_{2}-F_{2}^{2}$. Como $E 1 \cdot G_{1}-F_{1}^{2}=1$, temos que

$$
E 2 \cdot G_{2}-F_{2}^{2}=1
$$

Mas, como

$$
I_{X(q)}(v)=\left(\begin{array}{ll}
v_{x} & v_{y}
\end{array}\right)\left(\begin{array}{cc}
E_{2} & F_{2} \\
F_{2} & G_{2}
\end{array}\right)\left(\begin{array}{l}
v_{x} \\
v_{y}
\end{array}\right),
$$

temos que $E 2 \cdot G_{2}-F_{2}^{2}$ é o determinante da matriz associada a $I_{X(q)}$. E, como este determinante é igual a $\lambda_{1}(q) \cdot \lambda_{2}(q)$, onde $\lambda_{1}(q)$ e $\lambda_{2}(q)$ são dois autovalores da matriz, temos que

$$
\lambda_{1}(q) \cdot \lambda_{2}(q)=1
$$

Finalmente, se $X$ é uma isometria, então o Corolário B.1 nos diz que $E_{2}=E_{1}=1$, $F_{2}=F_{1}=0$ e $G_{2}=G_{1}=1$. Então, os dois autovalores, $\lambda_{1}(q)$ e $\lambda_{2}(q)$, da matriz associada com $I_{X(q)}$ são ambos iguais a 1. Esta observação nos diz que, se $X$ é uma isometria, então $X$ é também conformal e equiareal. Reciprocamente, se $X$ é conformal e equiareal, então o fato que $\lambda_{1}(q)=\lambda_{2}(q)$ e $\lambda_{1}(q) \cdot \lambda_{2}(q)=1$ implica que $\lambda_{1}(q)=\lambda_{2}(q)=1$. Então, se $X$ é conformal e equiareal, então $X$ é uma isometria.

Não considerando isometrias, qualquer outro tipo de parametrização $X$ impõe alguma distorção a $U$ quando mapeia $U$ para $X(U)$. Informalmente, isso significa que $X$ estica ou encolhe $U$ localmente, distorcendo ângulo e/ou área. Vamos considerar o que acontece com o ponto $X(u, v)$ em $X(U)$ quando variamos $(u, v)$ em um pequeno parâmetro no domínio $U$. Em particular, denotamos esse parâmetro infinitesimal por $\Delta u, \Delta v$. Então, nosso novo ponto na superfície $X(u+\Delta u, v+\Delta v)$ é aproximadamente dado pela expansão de primeira ordem de Taylor $\widetilde{X}$ de $X$, em torno de $(u, v)$. Ou seja,

$$
\widetilde{X}(u+\Delta u, v+\Delta v)=X(u, v)+X_{u}(u, v) \cdot \Delta u+X_{v}(u, v) \cdot \Delta v .
$$

A expressão acima leva todos os pontos da vizinhança de $(u, v)$ para o plano tangente afim de $S$ em $p=X(u, v)$, transformando círculos em torno de $(u, v)$ em elipses em torno de $p$, como está ilustrado na Figura B.5.

De fato, podemos reescrever a equação (B.18) como

$$
\widetilde{X}(u+\Delta u, v+\Delta v)=p+J(X)(u, v) \cdot\left(\begin{array}{c}
\Delta u \\
\Delta v
\end{array}\right)
$$




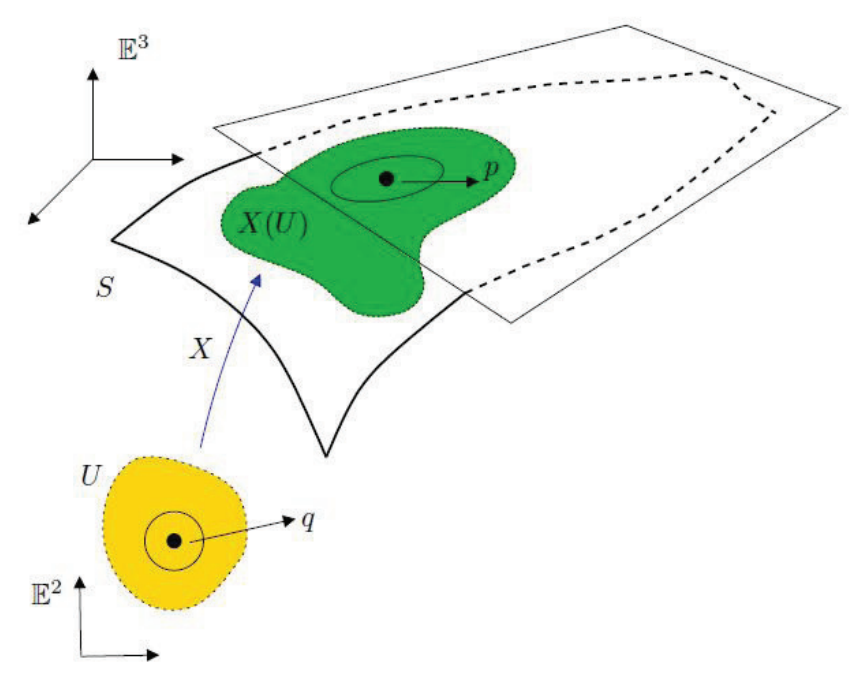

Figura B.5: Um círculo em torno de $q=(u, v)$ é levado por $X$ em uma elipse em torno de $p=X(q)$.

onde

$$
J(X)(u, v)=\left(\begin{array}{ll}
\frac{\partial x}{\partial u}(u, v) & \frac{\partial x}{\partial v}(u, v) \\
\frac{\partial y}{\partial u}(u, v) & \frac{\partial y}{\partial v}(u, v) \\
\frac{\partial z}{\partial u}(u, v) & \frac{\partial z}{\partial v}(u, v)
\end{array}\right)
$$

é a matriz Jacobiana de $(d X)_{(u, v)}$. Usando a decomposição em valores singulares (SVD) de $J(X)(u, v)$ (veja Gallier (2011)), temos

$$
J(X)(u, v)=V D W^{t}=V\left(\begin{array}{cc}
\sigma_{1}(u, v) & 0 \\
0 & \sigma_{2}(u, v) \\
0 & 0
\end{array}\right) W^{t}
$$

onde $\sigma_{1}(u, v) \geq \sigma_{2}(u, v)>0$ são os valores singulares de $J(X)(u, v)$ e $V \in M_{3}(\mathbb{R})$ e $W \in M_{2}(\mathbb{R})$ são as matrizes ortonormais. Usando a decomposição SVD, a transformação $J(X)(u, v) \cdot(\Delta u \Delta v)^{t}$ pode ser dividida em três:

1. A transformação linear associada à matriz $W^{t}$ é uma rotação que alinha a coluna de vetores $w_{1}$ e $w_{2}$ da matriz $W$ com os $v$ - e $u$-eixos das coordenadas locais do sistema de $\widetilde{X} \operatorname{em} U$.

2. A transformação linear associada à matriz $D$, que escala por um fator $\sigma_{1}(u, v)$ na $u$ direção e por um fator $\sigma_{2}(u, v)$ na $v$-direção, é a única responsável pela distorção.

3. A transformação linear associada à matriz $V$ é também uma rotação que mapeia os vetores unidades em $\mathbb{R}^{2}$ com coordenadas $(1,0)$ e $(0,1)$ para as duas primeiras colunas de vetores $v_{1}$ e $v_{2}$ de $V$ em $T_{X(u, v)} S$. 
A Figura B.6 ilustra estas três transformações. Como um resultado dos três passos descritos acima, temos que a transformação linear associada com $J(X)(u, v)$, isto é, o diferencial $(d X)_{(u, v)}$, mapeia a base ortonormal $\left\{w_{1}, w_{2}\right\}$ em $\mathbb{R}^{2}$ para a base ortonormal $\left\{\sigma_{1}(u, v)\right.$. $\left.v_{1}, \sigma_{2}(u, v) \cdot v_{2}\right\}$ em $T_{X(u, v)} S$. Isso significa que a função afim $\tilde{X}$ mapeia qualquer círculo em $U$ de raio $r$ e centro $(u, v)$ para uma elipse com semi-eixos $r \cdot \sigma_{1}(u, v)$ e $r \cdot \sigma_{2}(u, v)$ e centro $p=X(u, v)$ em um plano tangente afim de $S$ em $p$.

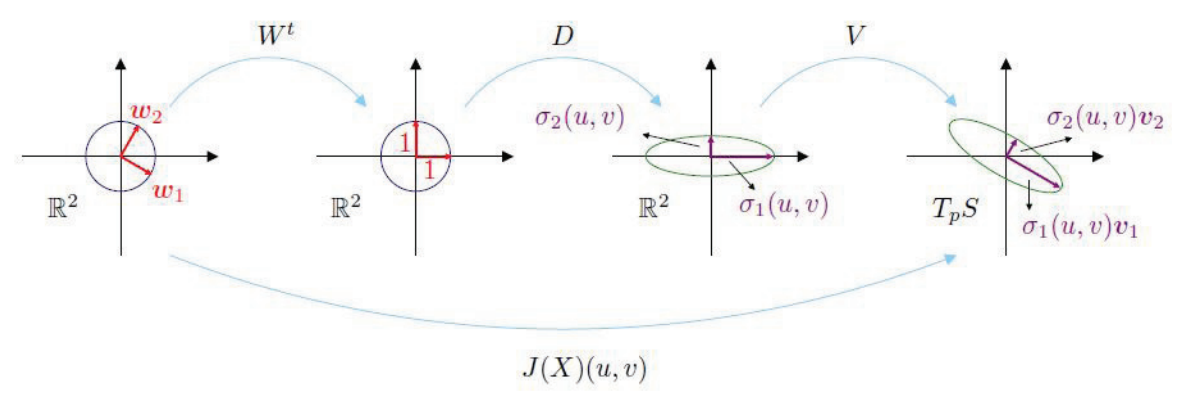

Figura B.6: Interpretação geométrica da transformação linear definida por $J(X)(u, v)$.

A transformação de círculos em elipses é chamada de distorção métrica local de uma parametrização $X$, que nos mostra como $X$ se comporta localmente em torno de um ponto $(u, v)$ de $U$. Além disso, toda informação sobre a distorção métrica local é dada pelos valores singulares, $\sigma_{1}(u, v)$ e $\sigma_{2}(u, v)$, que são os autovalores da matriz associada com a Primeira Forma Fundamental $I_{p}$ de $S$ em $p=X(u, v)$. De fato, lembre-se que, se $A$ é uma matriz qualquer, então os valores singulares de $A$ são as raízes quadradas dos autovalores da matriz $A^{t} A$ (veja Gallier (2011)). Como a matriz associada com $I_{p}$ é igual a $(J(X)(u, v))^{t} J(X)(u, v)$, temos que $\sigma_{i}(u, v)=\sqrt{\lambda_{i}(u, v)}$, para $i=1,2$.

Como $\sigma_{1}(u, v) \geq \sigma_{2}(u, v)>0$, temos que $\sigma_{1}(u, v)=\sigma_{2}(u, v)$ se, e somente se, $\lambda_{1}(u, v)=\lambda_{2}(u, v)$. Então, os valores singulares de $J(X)(u, v)$ nos dizem se $X$ é conformal, equiareal, isométrico, ou nenhum destes. Em particular, eles nos dão uma interpretação intuitiva das condições para mapas conformais e equiareais: se $\sigma_{1}(u, v)=\sigma_{2}(u, v)$, então $J(X)(u, v)$ é simplismente uma rotação mais uma escala uniforme, o que significa que $X$ não distorce ângulos em torno de $(u, v)$. Do mesmo modo, se $\sigma_{1}(u, v) \cdot \sigma_{2}(u, v)=1$, então a área do círculo de parâmetro em torno do domínio é igual à área correspondente à área da elipse no plano tangente, o que significa que $X$ preserva área em torno de $u, v$.

\section{B.7 Superfícies simpliciais}

Definição B.13. Sejam $v_{0}, \ldots, v_{d}$ quaisquer $d+1$ pontos linearmente independentes em $\mathbb{E}^{n}$, onde d é um inteiro não negativo. O simplexo $\sigma$, abrangido pelos pontos $v_{0}, \ldots, v_{d}$, é o casco 
convexo desses pontos, e é denotado por

$\left[v_{0}, \ldots, v_{d}\right]$

Os pontos $v_{0}, \ldots, v_{d}$ são os vértices de $\sigma$. A dimensão $\operatorname{dim}(\sigma)$ de $\sigma$ é $d$ e $\sigma$ é chamado um $d$-simplexo.

Em $\mathbb{E}^{n}$, o maior número de pontos linearmente independentes é $n+1$, e temos simplexos de dimensões $0,1, \ldots, n$. Um 0 -simplexo é um ponto, um 1 -simplexo é um segmento de reta, um 2-simplexo é um triângulo, um 3-simplexo é um tetraedro e o casco convexo que é um subconjunto não vazio de vértices de um simplexo é também um simplexo.

Definição B.14. Seja $\sigma=\left[v_{0}, \ldots, v_{d}\right]$ um $d$-simplexo em $\mathbb{E}^{n}$. Uma face de $\sigma$ é um simplexo medido por um subconjunto não vazio $\left\{v_{0}, \ldots, v_{d}\right\}$. Se esse subconjunto é próprio então a face é chamada de face própria. Uma face de $\sigma$ que é um k-simplexo é chamada de $k$-face. O limite combinatorial de $\sigma$, denotado por bd $(\sigma)$, é a união de todas as faces próprias de $\sigma$. O limite interior de $\sigma$, denotado por int $(\sigma)$, é o conjuto $\sigma-b d(\sigma)$.

Simplexos são usados como módulos para a definição de complexos simpliciais, que são os objetos mais gerais que podemos construir a partir dos simplexos. Complexos simpliciais podem ser construídos pela união de simplexos ao longo de suas faces comuns. Uma superfície simplicial é um tipo particular de complexo simplicial, que é composta de vértices, arestas e triângulos. Mais formalmente, temos a seguinte definição:

Definição B.15. Um complexo simplicial $\mathcal{K}$ em $\mathbb{E}^{n}$ é uma coleção finita de simplexos em $\mathbb{E}^{n}$ tal que

1. se um simplexo está em $\mathcal{K}$, então suas faces estão em $\mathcal{K}$;

2. se $\sigma, \tau \in \mathcal{K}$ são simplexos tais que $\sigma \cap \tau \neq \emptyset$, então $\sigma \cap \tau$ é uma face de $\sigma$ e de $\tau$.

A dimensão $\operatorname{dim}(\mathcal{K})$ de $\mathcal{K}$ é a dimensão do maior simplexo em $\mathcal{K}$, isto é,

$$
\operatorname{dim}(\mathcal{K})=\max \{\operatorname{dim}(\sigma) \mid \sigma \in \mathcal{K}\}
$$

Nos referimos a um complexo simplicial $d$-dimensional simplesmente como um $d$-complexo. O conjunto que consiste na união de todos os pontos do simplexos de $\mathcal{K}$ é chamado de espaço subjacente de $\mathcal{K}$ e é denotado por $|\mathcal{K}|$.

A Figura B.7 mostra três conjuntos de simplexos em $\mathbb{E}^{2}$. O conjunto (a) não é um complexo simplicial pois uma aresta e um vértice do limite (combinatório) de um 2-simplexo estão faltando. O conjunto (b) contém dois simplexos que se cruzam entre si, mas o cruzamento não é uma face de outro simplexo. Por isso também não é um complexo simplicial. O conjunto (c) é um complexo simplicial, uma vez que satisfaz as condições (1) e (2) de Definição B.15. 


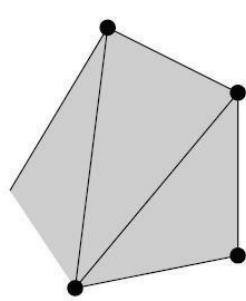

(a)

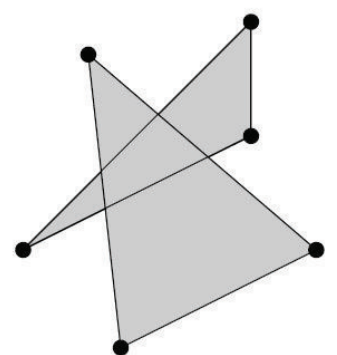

(b)

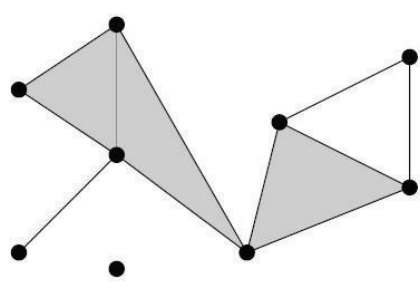

(c)

Figura B.7: Coleção de simplexos em $\mathbb{E}^{2}$. Apenas a figura (c) é um complexo simplicial.

Definição B.16. Seja $\mathcal{K}$ um complexo simplicial em $\mathbb{E}^{n}$. Então, para qualquer simplexo $\sigma$ em $\mathcal{K}$, definimos os conjuntos

$$
\text { st }(\sigma, \mathcal{K})=\{\tau \in \mathcal{K} \mid \exists \eta \text { em } \mathcal{K} \text { tal que } \sigma \text { é uma face de } \eta \text { e } \tau \text { é uma face de } \eta\}
$$

$e$

$$
l k(\sigma, \mathcal{K})=\{\tau \in \mathcal{K} \mid \tau \text { está em } s t(\sigma, \mathcal{K}) \text { e } \tau \text { e } \sigma \text { não tem faces em comum }\}
$$

chamados estrela e vizinhança de $\sigma$ em $\mathcal{K}$, respectivamente. Note que o conjunto $\operatorname{st}(\sigma, \mathcal{K})$ é composto por $\sigma$, todos os simplexos de $\mathcal{K}$ que tem $\sigma$ como uma face, e todos os simplexos de $\mathcal{K}$ que são faces de algum simplexo que tem $\sigma$ como uma face. Por sua vez, o conjunto $l k(\sigma, K)$ é composto de todos os simplexos em st $(\sigma, K)$ que não contêm $\sigma$ como uma face. Além disso, ambos conjuntos $l k(\sigma, K)$ e st $(\sigma, K)$ são complexos simpliciais, e st $(\sigma, K)$ é sempre não vazio.

A figura B.8 ilustra o conjunto $\operatorname{st}(\sigma, K)$ e $\operatorname{lk}(\sigma, K)$ da Definição B.16.

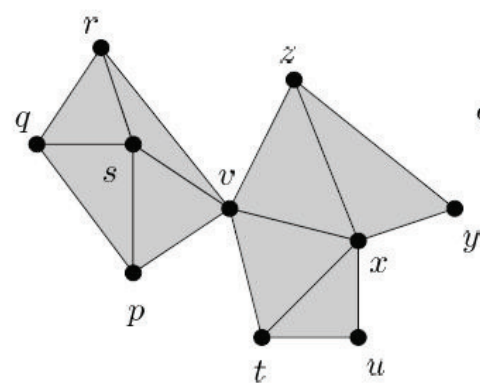

(a)

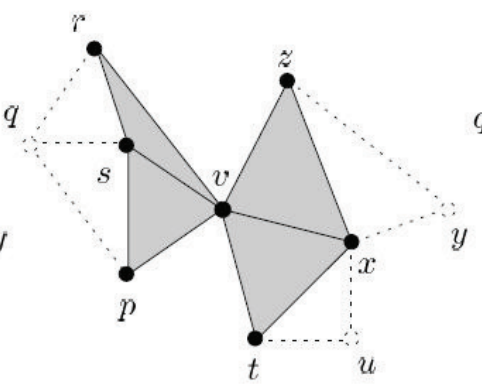

(b)

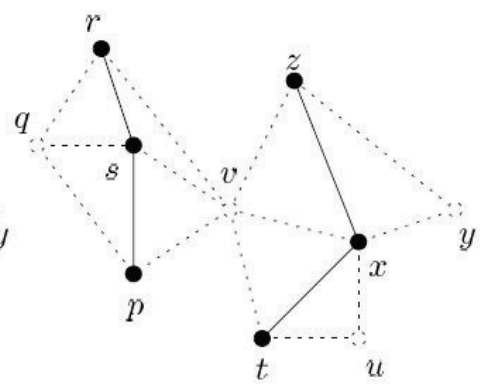

(c)

Figura B.8: Um complexo simplicial $K$ (a), uma estrela do vértice $v, \operatorname{st}(v, K)$

(b) e uma vizinhança do vértice $v, 1 \mathrm{k}(v, K)$ (c).

Definição B.17. Seja $\mathcal{K}$ um complexo simplicial em $\mathbb{E}^{n}$. Para cada inteiro $i$, com $0 \leq i \leq$ $\operatorname{dim}(\mathcal{K})$, definimos $\mathcal{K}^{((i))}$ como um complexo simplicial constituído de todos os $i$-simplexos de 
$\mathcal{K}$. Além disso, se $\mathcal{L}$ é um complexo simplicial em $\mathbb{E}^{m}$, então um mapa $f: \mathcal{K}^{((0))} \rightarrow \mathcal{L}^{((0))}$ é chamado de mapa simplicial sempre que $\left[v_{0}, \ldots, v_{d}\right]$ é um simplexo em $\mathcal{K}$, ou seja, $\left[f\left(v_{0}\right), \ldots, f\left(v_{d}\right)\right]$ é um simplexo em $\mathcal{L}$. Um mapa simplicial é um isomorfismo simplicial se é um mapa bijetor e sua inversa também é um mapa simplicial. Finalmente, se existir um isomorfismo simplicial de $\mathcal{K}$ para $\mathcal{L}$, dizemos que $\mathcal{K}$ e $\mathcal{L}$ são isomórficos simpliciais.

Definição B.18. Um 2-complexo $\mathcal{K}$ em $\mathbb{R}^{n}$ é chamado uma superfície simplicial sem borda se cada 1-simplexo de $\mathcal{K}$ é a face formada por dois simplexos de $\mathcal{K}$, e o espaço subjacente da vizinhança de cada 0 -simplexo de $\mathcal{K}$ é homeomórfico ao círculo unitário $\mathbb{S}^{1}=\left\{x \in \mathbb{E}^{2} \mid\|x\|=\right.$ 1\}. Se cada 1-simplexo de $\mathcal{K}$ é uma face de um ou dois simplexos de $\mathcal{K}$ e o espaço subjacente da vizinhança de cada 0 -simplexo de $\mathcal{K}$ é homeómorfico a qualquer círculo unitário $\mathbb{S}^{1}=$ $\left\{x \in \mathbb{E}^{2} \mid\|x\|=1\right\}$, ou ao segmento unitário fechado $\overline{\mathbb{B}}^{1}=\left\{x \in \mathbb{E}^{2} \mid\|x\| \leq 1\right\}$, dizemos que o complexo $\mathcal{K}$ é uma superfície simplicial com borda. O espaço subjacente da superfície simplicial (com ou sem borda) é chamado de superfície subjacente da superfície simplicial. A superfície subjacente é uma superfície topológica em $\mathbb{E}^{n}$.

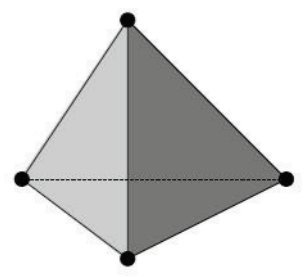

(a)

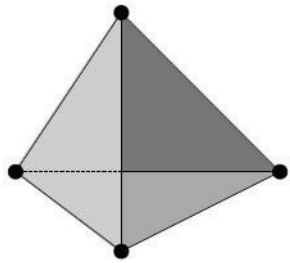

(b)

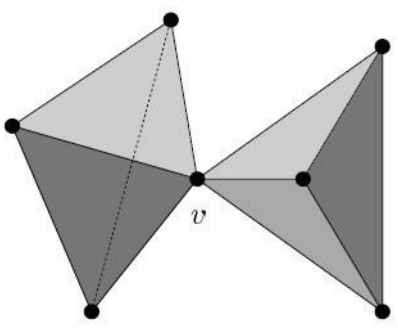

(c)

Figura B.9: Um complexo simplicial (a), uma estrela do vértice $v$ (b) e um vínculo do vértice $v$ (c).

A Figura B.9 ilustra a Definição B.18. O limite combinatorial de um 3-simplexo (isto é, um tetraedro) é uma superfície simplicial (Figura B.9(a)). Se removermos uma face a partir do limite combinatorial do tetraedro, obtemos uma superfície simplicial com fronteira (Figura B.9(b)). Mas, o 2-complexo $\mathcal{K}$ que consiste na união das fronteiras combinatorial da reunião de dois tetraedros em um vértice $v$ não é uma superfície simplicial (Figura B.9(c)). Isso porque $1 \mathrm{k}(v)$ em $\mathcal{K}$ não é homeomórfico a $\mathbb{S}^{1}$ ou $\overline{\mathrm{B}}^{1}$.

\section{B.8 Mudança de variáveis}

Definição B.19. Um subconjunto limitado $U$ de $\mathbb{E}^{n}$ é dito de medida nula se para cada $\epsilon \in \mathbb{R}$, com $\epsilon>0$, há uma coleção finita $\mathcal{F}$ de retângulos generalizados em $\mathbb{E}^{n}$ que cobre a superfície de $U$, cuja soma dos volumes é menor que $\epsilon$, ou seja, se

$$
\mathcal{F}=\left\{I_{1}, \ldots, I_{m}\right\}
$$


onde $I_{i}=\left[a_{i_{1}}, b_{i_{1}}\right] \times \ldots \times\left[a_{i_{m}}, b_{i_{m}}\right]$ e $a_{i_{1}}, b_{i_{1}} \in \mathbb{E}$, com $a_{i_{1}} \leq b_{i_{1}}$, para cada $i, j \in\{1, \ldots, m\}$, então

$$
U \subseteq \bigcup_{i=1}^{m} I_{i} \quad e \quad \sum_{i=1}^{m} \operatorname{vol}\left(I_{i}\right)<\epsilon .
$$

Definição B.20. Um subconjunto limitado $D$ de $\mathbb{E}^{n}$ é dito ser um domínio de Jordan se seu limite tem medida nula.

Definição B.21. Seja $U$ um subconjunto aberto de $\mathbb{E}^{n}$. Uma $\mathcal{C}^{1}$-função (função da classe $\mathcal{C}^{1}$ )

$$
\phi: U \rightarrow \mathbb{E}^{n}
$$

é chamada de uma mudança de variáveis suave se valem as seguintes propriedades:

1. A função $\phi$ é injetiva.

2. Para cada ponto $x \in U$, a matriz Jacobiana $J_{\phi(x)}$ de d $\phi$ em $x$ é invertível.

Teorema B.6. Suponha que a função $\phi: U \rightarrow \mathbb{E}^{n}$ é uma mudança de variáveis suave sobre o subconjunto aberto $U$ de $\mathbb{E}^{n}$. Seja $D$ um domínio aberto de Jordan tal que $K=D \cup b d(D)$ está contido em $U$. Então, o conjunto aberto $\phi(K)$ é um domínio de Jordan com a propriedade que, para qualquer função contínua $f: \phi(K) \rightarrow \mathbb{E}$, vale a seguinte integral de transformação:

$$
\int_{\phi(K)} f(x) d x=\int_{K}(f \circ \phi)(u)|\operatorname{det}(J(\phi)(u))| d u .
$$

A prova do Teorema B.6 pode ser encontrada em Fitzpatrick (2006).

Sejam $t_{1}=[p, q, r]$ e $t_{2}=\left[p^{\prime}, q^{\prime}, r^{\prime}\right]$ dois triângulos em $\mathbb{E}^{2}$. Sabemos que existe um mapa afim único $g: \mathbb{E}^{2} \rightarrow \mathbb{E}^{2}$ que leva de $t_{1}$ para $t_{2}$ e tal que $g(p)=p^{\prime}, g(q)=q^{\prime}$ e $g(r)=r^{\prime}$. Podemos escrever $g$ como

$$
g(x)=A x+b, \text { para todo } x \in \mathbb{E}^{2},
$$

onde $A \in M_{2}(\mathbb{R})$ é uma matriz quadrada de ordem 2 e $b \in \mathbb{R}^{2}$ é um vetor coluna. O mapa $g$ é bijetivo e continuamente diferenciável, sua inversa $g^{-1}$ é também continuamente diferenciável, isto é, $g$ é uma $\mathcal{C}^{1}$-função.

Para calcular $g$, geralmente calculamos dois mapas afim, $g_{1}$ e $g_{2}$, onde $g_{1}$ e $g_{2}$ assumem o triângulo canônico $t_{e}=[(0,0),(1,0),(0,1)]$ para os triângulos $t_{1}$ e $t_{2}$, respectivamente, e são tais que

$$
\begin{gathered}
g_{1}((0,0))=p, g_{1}((1,0))=q, g_{1}((0,1))=r, \\
g_{2}((0,0))=p^{\prime}, g_{2}((1,0))=q^{\prime} \text { e } g_{2}((0,1))=r^{\prime} .
\end{gathered}
$$


Além disso,

$$
\begin{gathered}
g_{1}(x)=A_{1} x+b_{1}=\left(\begin{array}{ll}
a_{1} & b_{1} \\
c_{1} & d_{1}
\end{array}\right)+\left(\begin{array}{c}
e_{1} \\
f_{1}
\end{array}\right) \mathrm{e} \\
g_{2}(x)=A_{2} x+b_{2}=\left(\begin{array}{ll}
a_{2} & b_{2} \\
c_{2} & d_{2}
\end{array}\right)+\left(\begin{array}{c}
e_{2} \\
f_{2}
\end{array}\right),
\end{gathered}
$$

onde $\left(a_{1}, c_{1}\right),\left(a_{2}, c_{2}\right),\left(b_{1}, d_{1}\right)$ e $\left(b_{2}, d_{2}\right)$ são as coordenadas dos vetores $p q, p^{\prime} q^{\prime}, p r$ e $p^{\prime} r^{\prime}$, respectivamente, $\left(e_{1}, f_{1}\right)$ e $\left(e_{2}, f_{2}\right)$ são as coordenadas dos vértices $p$ e $p^{\prime}$ de $t_{1}$ e $t_{2}$, respectivamente. Finalmente, temos $g=g_{2} \circ g_{1}^{-1}$, onde $g_{1}^{-1}(x)=A_{1}^{-1} x+A_{1}^{-1} b_{1}$ e $A_{1}^{-1}$ é a inversa e A.

A Figura B.10 ilustra o mapa $g=g_{2} \circ g_{1}^{-1}$ que leva $t_{1}$ em $t_{2}$.

De acordo com a regra da cadeia, a matriz Jacobiana $J_{g(x)}$ de $d g$ em $x \in \mathbb{E}^{2}$ é igual a $J_{g_{2}\left(g_{1}^{-1}(x)\right)} \cdot J_{g_{1}^{-1}(x)}$, onde $J_{g_{2}\left(g_{1}^{-1}(x)\right)}$ é a matriz Jacobiana de $d g_{2}$ em $g_{1}^{-1}(x)$, e $J_{g_{1}^{-1}(x)}$ é a matriz Jacobiana de $d g_{1}^{-1}$ em $x$. Como $g_{1}^{-1}$ e $g_{2}$ são mapas afins, temos que $J_{g_{1}^{-1}(y)}=A_{1}^{-1}$ e $J_{g_{2}(y)}=A_{2}$, para todo $y \in \mathbb{E}^{2}$. Além disso, como $g_{1}^{-1}$ e $g_{2}$ são bijetivos, $A_{1}^{-1}$ e $A_{2}$ são invertíveis, o que significa que $A_{2} \cdot A_{1}^{-1}$ é invertível e que $g$ é uma mudança suave de variáveis (veja Definição B.21).

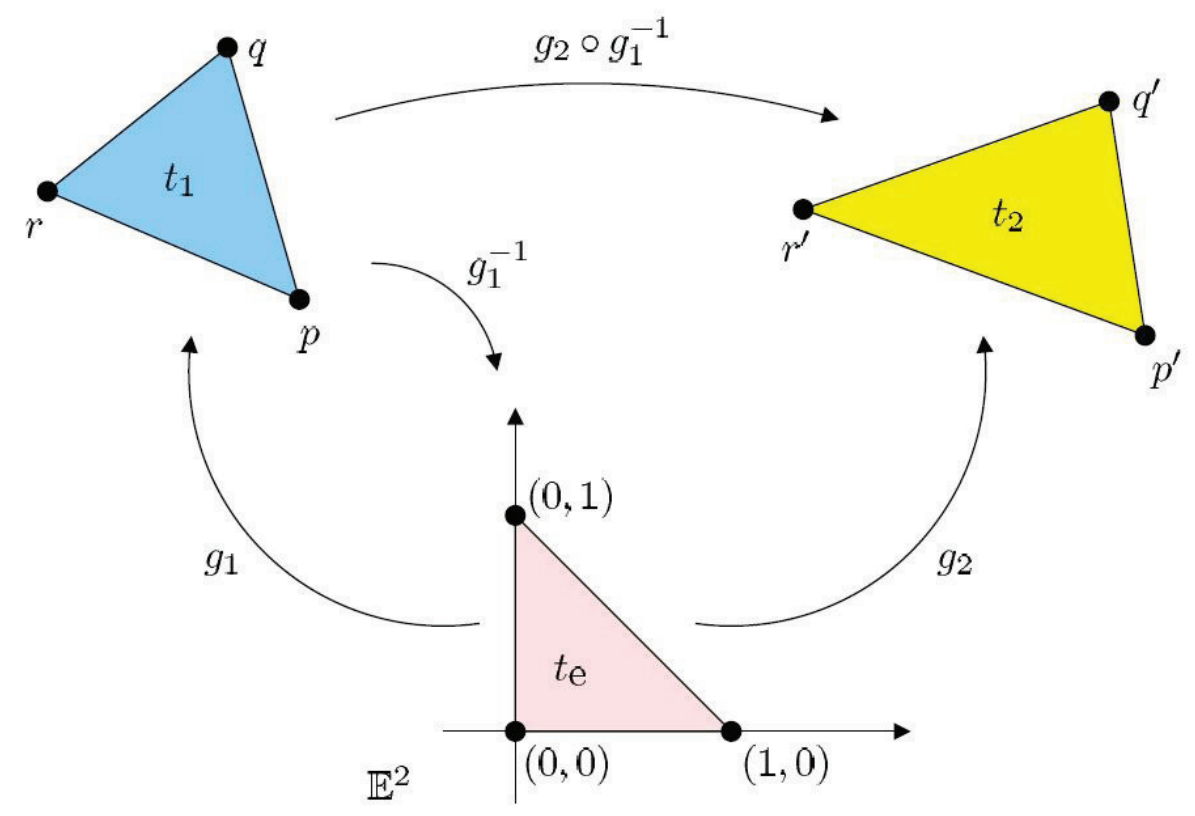

Figura B.10: Construção do mapa $g=g_{2} \circ g_{1}^{-1}$.

Seja $f: t_{2} \rightarrow \mathbb{E}$ uma função contínua definida em $t_{2}$. Suponha que queremos calcular a integral

$$
\int_{t_{2}} f(x) d x
$$


Se temos $D=\operatorname{int}\left(t_{1}\right)$, então $D$ é um domínio de Jordan, já que bd $(D)=\operatorname{bd}\left(\operatorname{int}\left(t_{1}\right)\right)=$ $\operatorname{bd}\left(t_{1}\right)$ e $\operatorname{bd}\left(t_{1}\right)$ tem medida nula. Então, tomando $U=\mathbb{E}^{2}, D=\operatorname{int}\left(t_{1}\right)$ e $\phi=g$ no Teorema B.6, temos $K=t_{1}, \phi(K)=t_{2}$ e

$$
\begin{aligned}
\int_{t_{2}} f(x) d x & =\int_{t_{1}}(f \circ g)(y)|\operatorname{det}(J(g)(y))| d y \\
& =\int_{t_{1}}(f \circ g)(y)\left|\operatorname{det}\left(A_{2} \cdot A_{1}^{-1}\right)\right| d y \\
& =\int_{t_{1}}(f \circ g)(y)\left|\operatorname{det}\left(A_{2}\right)\right| \cdot\left|\operatorname{det}\left(A_{1}^{-1}\right)\right| d y \\
& =\int_{t_{1}}(f \circ g)(y) \frac{\left|\operatorname{det}\left(A_{2}\right)\right|}{\left|\operatorname{det}\left(A_{1}\right)\right|} d y .
\end{aligned}
$$

\section{Como}

$$
\frac{\left|\operatorname{det}\left(A_{2}\right)\right|}{\left|\operatorname{det}\left(A_{1}\right)\right|}
$$

não depende de $y$, obtemos

$$
\int_{t_{2}} f(x) d x=\frac{\left|\operatorname{det}\left(A_{2}\right)\right|}{\left|\operatorname{det}\left(A_{1}\right)\right|} \cdot \int_{t_{1}}(f \circ g)(y) d y=\frac{\Delta\left(t_{2}\right)}{\Delta\left(t_{1}\right)} \cdot \int_{t_{1}}(f \circ g)(y) d y,
$$

onde $\Delta\left(t_{1}\right)=\frac{\operatorname{det} A_{1}}{2}$ e $\Delta\left(t_{2}\right)=\frac{\operatorname{det} A_{2}}{2}$ são as áreas dos triângulos $t_{1}$ e $t_{2}$, respectivamente. 



\section{APÊNDICE}

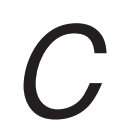

\section{Demonstrações dos teoremas do Apêndice B}

Lema B.1. Sejam $S$ uma superfície e $X: U \rightarrow X(U) \subseteq S$ uma parametrização na qual a imagem contém o ponto p. Então, existe um subconjunto aberto $V$ de $U$ contendo o ponto $q$ e uma projeção ortogonal $\pi: \mathbb{E}^{3} \rightarrow \mathbb{R}^{2}$ sobre alguma das três coordenadas planas de $\mathbb{R}^{3}$ tal que $W=(\pi \circ X)(V)$ é aberto em $\mathbb{E}^{2}$ e tal que $\pi \circ X: V \rightarrow W$ é um difeomorfismo.

Prova: Podemos escrever as componentes de uma parametrização $X$ como

$$
X(u, v)=(x(u, v), y(u, v), z(u, v))
$$

Como $(d X)_{q}$ é injetor, sua matriz associada $J_{X(a)}$, que é a matriz Jacobiana de $(d X)_{q}$,

$$
J_{X(a)}=\left(\begin{array}{ll}
\frac{\partial x}{\partial u}(q) & \frac{\partial x}{\partial v}(q) \\
\frac{\partial y}{\partial u}(q) & \frac{\partial y}{\partial v}(q) \\
\frac{\partial z}{\partial u}(q) & \frac{\partial z}{\partial v}(q)
\end{array}\right)
$$

tem posto dois. Então, ela tem um menor não nulo de ordem dois. Sem perda de generalidade, suponha que esse menor é formado exatamente pelas duas primeiras linhas da matriz. Seja $\pi: \mathbb{E}^{3} \rightarrow \mathbb{E}^{3}$ uma projeção ortogonal sobre o plano $x y$, que é dada por $\pi(x, y, z)=(x, y)$. Então, $d(\pi \circ X)_{q}: \mathbb{R}^{2} \rightarrow \mathbb{R}^{2}$ é dado por

$$
d(\pi \circ X)_{q}=(d \pi)(X(q)) \circ(d X)_{q}=\pi \circ(d X)_{q}
$$


já que a matriz Jacobiana $J_{\pi(X(q))}$ do diferencial $(d \pi)(X(q))$ da projeção ortogonal $\pi$ em $q$ é

$$
\left(\begin{array}{lll}
1 & 0 & 0 \\
0 & 1 & 0
\end{array}\right)
$$

Então, para qualquer $v=\left(v_{x}, v_{y}\right) \in \mathbb{R}^{2}$, obtemos

$$
\begin{aligned}
d(\pi \circ X)_{q}(v) & =\pi \circ(d X)_{q}(v) \\
& =\pi\left(v_{x} \cdot \frac{\partial x}{\partial u}(q)+v_{y} \cdot \frac{\partial x}{\partial v}(q), v_{x} \cdot \frac{\partial y}{\partial u}(q)+v_{y} \cdot \frac{\partial y}{\partial v}(q), v_{x} \cdot \frac{\partial z}{\partial u}(q)+v_{y} \cdot \frac{\partial z}{\partial v}(q)\right) \\
& =\left(\begin{array}{cc}
\frac{\partial x}{\partial u}(q) & \frac{\partial x}{\partial v}(q) \\
\frac{\partial y}{\partial u}(q) & \frac{\partial y}{\partial v}(q)
\end{array}\right) \cdot\left(\begin{array}{c}
v_{x} \\
v_{y}
\end{array}\right) .
\end{aligned}
$$

Então, o mapa linear $d(\pi \circ X)_{q}$ é bijetor. Para finalizar, voltamos ao Teorema da Função Inversa, que nos diz que existe um conjunto $V \subset U$ tal que a restrição de $\pi \circ X$ para $V$ é um difeomorfismo.

Teorema B.2. Toda mudança de coordenadas é um difeomorfismo.

Prova: Seja $S$ uma surperfície, e sejam $X_{i}: U_{i} \rightarrow X_{i}(U) \subseteq S$, para $i=1,2$, duas parametrizações de $S$ tal que $\Omega=X_{1}\left(U_{1}\right) \cap X_{2}\left(U_{2}\right)$ é não vazio. Considere a mudança de coordenadas $h=X_{2}^{-1} \circ X_{1}: X_{1}^{-1}(\Omega) \rightarrow X_{2}^{-1}(\Omega)$. Como $h$ é um mapa bijetor e sua inversa é outra mudança de coordenadas, é suficiente mostrar que $h$ é diferenciável em cada ponto do domínio $X_{1}^{-1}(\Omega)$. Tome $q_{1} \in X_{1}^{-1}(\Omega)$ e $q_{2}=h\left(q_{1}\right) \in X_{2}^{-1}(\Omega)$. Aplicando o Lema B.1, encontramos um conjunto aberto $V$, com $V \subset X_{2}^{-1}(\Omega)$ e $q_{2} \in V$, e uma projeção ortogonal (sobre o plano $x y$ ),

$$
\pi: \mathbb{E}^{3} \rightarrow \mathbb{E}^{2}
$$

tal que

$$
\pi \circ X_{2}: V \rightarrow W, \operatorname{com} W=\left(\pi \circ X_{2}\right)(V)
$$

é um difeomorfismo. Agora, pelos mapas $X_{2}^{-1}$ e $\left(\pi \circ X_{2}\right)^{-1} \circ \pi$ coincidirem em $X_{2}(V)$, a restrição

$$
h_{\mid h^{-1}(V)}=h^{-1}(V) \rightarrow V
$$

de $h$ para $h^{-1}(V)$ é tal que

$$
h_{\mid h^{-1}(V)}=\left(X_{2}^{-1} \circ X_{1}\right)_{\mid h^{-1}(V)}=\left(\left(\pi \circ X_{2}\right)^{-1} \circ \pi \circ X_{1}\right)_{\mid h^{-1}(V)} .
$$

Como $\left(\pi \circ X_{2}\right)$ é um homeomorfismo, e como $\pi$ e $X_{1}$ são diferenciáveis em $h^{-1}(V)$, a regra da cadeia implica que $h_{h^{-1}(V)}$ é diferenciável. Então, $h$ é diferenciável em $q_{1} \in h^{-1}(V)$. Como 
$q_{1}$ é um ponto arbitrário em $X_{1}^{-2}(\Omega)$, segue que $h$ é diferenciável. Como a inversa $h^{-1}$ de $h$ é $X_{1}^{-1} \circ X_{2}$, podemos usar o mesmo argumento para provar que $h^{-1}$ é também diferenciável. Portanto, o mapa $h$ é um difeomorfismo.

Lema B.2. Sejam $S$ uma superfície, $p \in S$ e $X: U \rightarrow X(U) \subseteq S$ uma parametrização de $S$ com $p \in X(U)$. Então,

$$
T_{p} S=(d X)_{X^{-1}(p)}\left(\mathbb{R}^{2}\right)
$$

Prova: Dado $w \in \mathbb{R}^{2}$, considere o segmento de reta $\beta:(-\varepsilon, \varepsilon) \rightarrow U$, para um $\varepsilon$ pequeno, tal que $\beta(t)=q+t \cdot w$, onde $q=X^{-1}(p)$. A curva $\beta$ verifica $\beta(0)=q$ e $\beta^{-1}(0)=w$. Então, se definimos $\alpha:(-\varepsilon, \varepsilon) \rightarrow S$ como $\alpha=X \circ \beta$, temos que $\alpha(0)=p$ e $\alpha^{\prime}(0)=(X \circ \beta)^{\prime}(0)=$ $(d X)_{q}(w)$. Então, $(d X)_{X^{-1}(p)}\left(\mathbb{R}^{2}\right) \subset T_{p} S$, com $\alpha$ tomando valores em $S$. Reciprocamente, considere um vetor $v$ tangente a $S$ em $p$. Por definição, existe uma curva $\alpha:(-\varepsilon, \varepsilon) \rightarrow S$, $\operatorname{com} \varepsilon>0$, tal que $\alpha(0)=p$ e $\alpha^{\prime}(0)=v$. Tomando $\varepsilon$ pequeno o suficiente, podemos supor, próximo da continuidade de $\alpha$, que $\alpha(-\varepsilon, \varepsilon)$ está contido em $X(U)$. Definimos uma curva em $U$ por $\beta=X^{-1} \circ \alpha$. Note que $\beta(0)=q$ e $\alpha=X \circ \beta$. Deste modo, $v=\alpha^{\prime}(0)=(X \circ \beta)^{\prime}(0)=$ $\left(d X_{q}\right)\left(\beta^{\prime}(0)\right)$, ou seja, qualquer vetor tangente a $S$ por $p$ encontra-se na imagem de $(d X)_{q}$.

Proposição B.1. Sejam $S$ uma superfície, $p \in \S, E, F, G$ são os coeficientes da Primeira Forma Fundamental na base na base $\left\{X_{u}, X_{v}\right\}$ de $T_{p} S$. Então

$$
\left\|X_{u} \times X_{v}\right\|=\left(E G-F^{2}\right)^{\frac{1}{2}}
$$

Prova: Lembre-se que, se $a, b, c$ e $d$ são vetores em $\mathbb{R}^{3}$, então

$$
(a \times b) \cdot(c \times d)=(a \cdot c)(b \cdot d)-(a \cdot d)(b \cdot c) .
$$

Aplicando este resultado para $\left\|X_{u} \times X_{v}\right\|^{2}=\left(X_{u} \times X_{v}\right) \cdot\left(X_{u} \times X_{v}\right)$, temos

$$
\left\|X_{u} \times X_{v}\right\|^{2}=\left(X_{u} \cdot X_{u}\right)\left(X_{v} \cdot X_{v}\right)-\left(X_{u} \cdot X_{v}\right)^{2}=E G-F^{2} .
$$




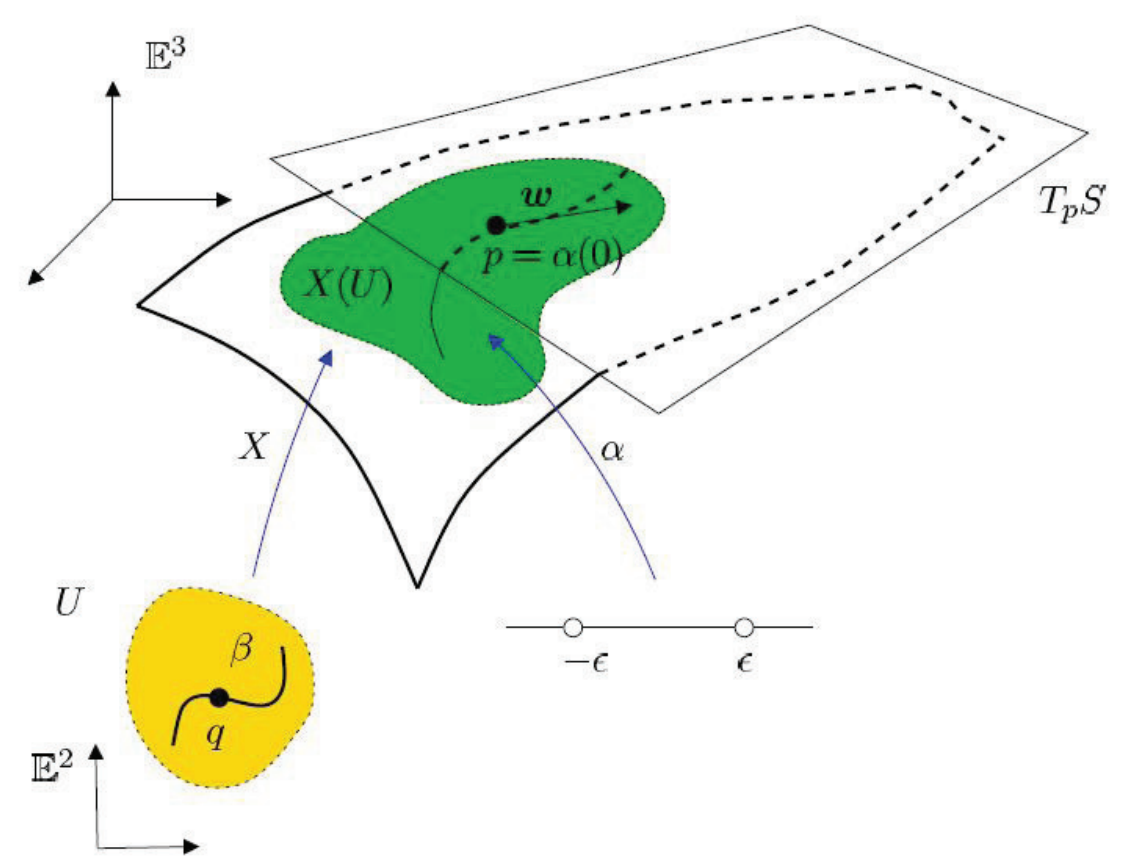

Figura C.1: Ilustração da prova do Lema B.2

Proposição B.2. Sejam E, F, G são os coeficientes da Primeira Forma Fundamental na base na base $\left\{X_{u}, X_{v}\right\}$ de $T_{p} S$ e ds é chamado elemento do comprimento do arco. Então, a expressão $d s^{2}=E d u^{2}+2 F d u d v+G d v^{2}$ não é alterada por uma reparametrização.

Prova: Sejam $S$ uma superfície, $X: U \rightarrow X(U) \subseteq S$ e $Y: V \rightarrow Y(V) \subseteq S$ parametrizações de $S$, e $\alpha:(-\varepsilon, \varepsilon) \rightarrow S$ uma curva regular de $S$ encontrando-se em $\Omega=X(U) \cap Y(V)$, onde $\varepsilon$ é um número positivo. Então

$$
\alpha(t)=X(u(t), v(t))=Y(\widetilde{u}(t), \widetilde{v}(t))
$$

para todo $t \in(-\varepsilon, \varepsilon)$. Note que $(\widetilde{u}(t), \widetilde{v}(t))=\left(Y^{-1} \circ X\right)(u(t), v(t))$. Se representamos o vetor tangente $\alpha^{\prime}(t)$ à curva $\alpha$ em $\alpha(t)$ com respeito a duas diferentes parametrizações de $S$, temos que

$$
\alpha^{\prime}(t)=X_{u} \frac{d u}{d t}+X_{v} \frac{d v}{d t}=Y_{\widetilde{u}} \frac{d \widetilde{u}}{d t}+Y_{\widetilde{v}} \frac{d \widetilde{v}}{d t}
$$

Agora, vamos escrever os coeficientes $E, F, G$ e $\widetilde{E}, \widetilde{F}, \widetilde{G}$ da Primeira Forma Fundamental de $S$ por $\alpha(t)$ com respeito a $X$ e $Y$, respectivamente. Primeiro, note que a mudança de coordenadas $Y^{-1} \circ X$ implica que

$$
X_{u}=Y_{\widetilde{u}} \frac{\partial \widetilde{u}}{\partial u}+Y_{\widetilde{v}} \frac{\partial \widetilde{v}}{\partial u} \quad \text { e } \quad X_{v}=Y_{\widetilde{u}} \frac{\partial \widetilde{u}}{\partial v}+Y_{\widetilde{v}} \frac{\partial \widetilde{v}}{\partial v}
$$


Então,

$$
\begin{aligned}
& E=\left\langle X_{u}, X_{u}\right\rangle_{\alpha(t)}=\widetilde{E}\left(\frac{\partial \widetilde{u}}{\partial u}\right)^{2}+2 \widetilde{F} \frac{\partial \widetilde{u}}{\partial u} \frac{\partial \widetilde{v}}{\partial u}+\widetilde{G}\left(\frac{\partial \widetilde{v}}{\partial u}\right)^{2} \\
& F=\left\langle X_{u}, X_{v}\right\rangle_{\alpha(t)}=\widetilde{E} \frac{\partial \widetilde{u}}{\partial u} \frac{\partial \widetilde{u}}{\partial v}+2 \widetilde{F}\left(\frac{\partial \widetilde{u}}{\partial u} \frac{\partial \widetilde{v}}{\partial v}+\frac{\partial \widetilde{u}}{\partial v} \frac{\partial \widetilde{v}}{\partial u}\right)+\widetilde{G} \frac{\partial \widetilde{v}}{\partial u} \frac{\partial \widetilde{v}}{\partial v} \\
& G=\left\langle X_{v}, X_{v}\right\rangle_{\alpha(t)}=\widetilde{E}\left(\frac{d \widetilde{u}}{d v}\right)^{2}+2 \widetilde{F} \frac{\partial \widetilde{u}}{\partial v} \frac{\partial \widetilde{v}}{\partial v}+\widetilde{G}\left(\frac{\partial \widetilde{v}}{\partial v}\right)^{2}
\end{aligned}
$$

Na matriz da Primeira Forma Fundamental, temos:

$$
\left(\begin{array}{cc}
E & F \\
F & G
\end{array}\right)=\left(\begin{array}{cc}
\frac{\partial \widetilde{u}}{\partial u} & \frac{\partial \widetilde{v}}{\partial u} \\
\frac{\partial \widetilde{u}}{\partial v} & \frac{\partial \widetilde{v}}{\partial v}
\end{array}\right)\left(\begin{array}{cc}
\widetilde{E} & \widetilde{F} \\
\widetilde{F} & \widetilde{G}
\end{array}\right)\left(\begin{array}{cc}
\frac{\partial \widetilde{u}}{\partial u} & \frac{\partial \widetilde{u}}{\partial v} \\
\frac{\partial \widetilde{v}}{\partial u} & \frac{\partial \widetilde{v}}{\partial v}
\end{array}\right)=J\left(Y^{-1} \circ X\right)^{t}\left(\begin{array}{cc}
\widetilde{E} & \widetilde{F} \\
\widetilde{F} & \widetilde{G}
\end{array}\right) J\left(Y^{-1} \circ X\right),
$$

onde $J\left(Y^{-1} \circ X\right)$ é a matriz Jacobiana do diferencial $\left(d\left(Y^{-1} \circ X\right)\right)_{(u, v)}$ de $(\widetilde{u}, \widetilde{v})=\left(Y^{-1} \circ\right.$ $X)(u, v)$.

Então,

$$
\left(\begin{array}{c}
\frac{d \widetilde{u}}{d t} \\
\frac{d \widetilde{v}}{d t}
\end{array}\right)=J\left(Y^{-1} \circ X\right)\left(\begin{array}{c}
\frac{d u}{d t} \\
\frac{d v}{d t}
\end{array}\right)
$$

A igualdade acima provém da equação matricial

$$
\begin{aligned}
d s^{2} & =\left(\begin{array}{ll}
d u & d v
\end{array}\right)\left(\begin{array}{cc}
E & F \\
F & G
\end{array}\right)\left(\begin{array}{c}
d u \\
d v
\end{array}\right) \\
& =\left(J\left(Y^{-1} \circ X\right)\left(\begin{array}{c}
d u \\
d v
\end{array}\right)\right)^{t}\left(\begin{array}{cc}
\widetilde{E} & \widetilde{F} \\
\widetilde{F} & \widetilde{G}
\end{array}\right)\left(\left(J\left(Y^{-1} \circ X\right)\left(\begin{array}{c}
d u \\
d v
\end{array}\right)\right)\right. \\
& =\left(\begin{array}{ll}
d \widetilde{u} & d \widetilde{v}
\end{array}\right)\left(\begin{array}{cc}
\widetilde{E} & \widetilde{F} \\
\widetilde{F} & \widetilde{G}
\end{array}\right)\left(\begin{array}{c}
d \widetilde{u} \\
d \widetilde{v}
\end{array}\right),
\end{aligned}
$$

que mostra que $d s$ é invariante com respeito à mudança de coordenadas $Y^{-1} \circ X$.

Proposição B.3. Sejam E, F, G são os coeficientes da Primeira Forma Fundamental na base na base $\left\{X_{u}, X_{v}\right\}$ de $T_{p} S$ e ds é chamado elemento do comprimento do arco. Então, a expressão

$$
\theta=\arccos \left(\frac{E \frac{d u}{d t} \frac{d v}{d s}+F\left(\frac{d u}{d t} \frac{d u}{d s}+\frac{d u}{d s} \frac{d v}{d t}\right)+G \frac{d v}{d t} \frac{d v}{d s}}{\left(E\left(\frac{d u}{d t}\right)^{2}+2 F \frac{d u}{d t} \frac{d v}{d t}+G\left(\frac{d v}{d t}\right)^{2}\right)^{\frac{1}{2}}\left(E\left(\frac{d u}{d s}\right)^{2}+2 F \frac{d u}{d s} \frac{d v}{d s}+G\left(\frac{d v}{d s}\right)^{2}\right)^{\frac{1}{2}}}\right)
$$

não é alterada por uma reparametrização.

Prova: Sejam $S$ uma superfície, $X: U \rightarrow X(U) \subseteq S$ e $Y: V \rightarrow Y(V) \subseteq S$ parametrizações de $S$ tal que $\Omega=X(U) \cap Y(V)$ é não vazio. Sejam $\alpha, \beta:(-\varepsilon, \varepsilon) \rightarrow S$ duas curvas regulares tais que

$$
\alpha(t)=X(u(t), v(t))=Y(\widetilde{u}(t), \widetilde{v}(t)) \quad \text { e } \quad \beta(s)=X(u(s), v(s))=Y(\widetilde{u}(s), \widetilde{s}(t)),
$$


para todo $t, s \in(-\varepsilon, \varepsilon)$. Suponha que $\alpha$ e $\beta$ encontram-se em $\Omega$ e que $\alpha(t)=\beta s$, para $t=t_{0}$ e $s=s_{0}$. Então

$$
(\widetilde{u}(t), \widetilde{v}(t))=\left(Y^{-1} \circ X\right)(u(t), v(t)) \quad \mathrm{e} \quad(\widetilde{u}(s), \widetilde{v}(s))=\left(Y^{-1} \circ X\right)(u(s), v(s)) .
$$

Temos que

$$
\begin{gathered}
\left(\begin{array}{c}
\frac{d \widetilde{u}}{d t} \\
\frac{d \widetilde{v}}{d t}
\end{array}\right)=J\left(Y^{-1} \circ X\right)\left(\begin{array}{c}
\frac{d u}{d t} \\
\frac{d v}{d t}
\end{array}\right),\left(\begin{array}{c}
\frac{d \widetilde{u}}{d s} \\
\frac{d \widetilde{v}}{d s}
\end{array}\right)=J\left(Y^{-1} \circ X\right)\left(\begin{array}{c}
\frac{d u}{d s} \\
\frac{d v}{d s}
\end{array}\right), \\
X_{u}=Y_{\widetilde{u}} \frac{\partial \widetilde{u}}{\partial u}+Y_{\widetilde{v}} \frac{\partial \widetilde{v}}{\partial u} \quad \text { e } \quad X_{v}=Y_{\widetilde{u}} \frac{\partial \widetilde{u}}{\partial v}+Y_{\widetilde{v}} \frac{\partial \widetilde{v}}{\partial v}
\end{gathered}
$$

Então,

$$
\begin{aligned}
& E=\left\langle X_{u}, X_{u}\right\rangle_{\alpha(t)}=\widetilde{E}\left(\frac{\partial \widetilde{u}}{\partial u}\right)^{2}+2 \widetilde{F} \frac{\partial \widetilde{u}}{\partial u} \frac{\partial \widetilde{v}}{\partial u}+\widetilde{G}\left(\frac{\partial \widetilde{v}}{\partial u}\right)^{2}, \\
& F=\left\langle X_{u}, X_{v}\right\rangle_{\alpha(t)}=\widetilde{E} \frac{\partial \widetilde{u}}{\partial u} \frac{\partial \widetilde{u}}{\partial v}+2 \widetilde{F}\left(\frac{\partial \widetilde{u}}{\partial u} \frac{\partial \widetilde{v}}{\partial v}+\frac{\partial \widetilde{u}}{\partial v} \frac{\partial \widetilde{v}}{\partial u}\right)+\widetilde{G} \frac{\partial \widetilde{v}}{\partial u} \frac{\partial \widetilde{v}}{\partial v}, \\
& G=\left\langle X_{v}, X_{v}\right\rangle_{\alpha(t)}=\widetilde{E}\left(\frac{d \widetilde{u}}{d v}\right)^{2}+2 \widetilde{F} \frac{\partial \widetilde{u}}{\partial v} \frac{\partial \widetilde{v}}{\partial v}+\widetilde{G}\left(\frac{\partial \widetilde{v}}{\partial v}\right)^{2} .
\end{aligned}
$$

onde $E, F, G$ e $\widetilde{E}, \widetilde{F}, \widetilde{G}$ são os coeficientes da Primeira Forma Fundamental de $S$ em $p=$ $\alpha\left(t_{0}\right)=\beta\left(t_{0}\right)$ com respeito a $X$ e $Y$, respectivamente. Substituindo $E, F$ e $G$ com o lado direito das equações acima no denominador da equação (C.1) e rearranjando os termos multiplicados por $\widetilde{E}, \widetilde{F}$ e $\widetilde{G}$, temos

$$
E \frac{d u}{d t} \frac{d u}{d s}+2 F\left(\frac{d u}{d t} \frac{d v}{d s}+\frac{d u}{d s} \frac{d v}{d t}\right)+G \frac{d v}{d t} \frac{d v}{d s}=\widetilde{E} \cdot \mu_{\widetilde{E}}+\widetilde{F} \cdot \mu_{\widetilde{F}}+\widetilde{G} \cdot \mu_{\widetilde{G}}
$$

onde

$$
\begin{gathered}
\mu_{\widetilde{E}}=\left(\frac{\partial \widetilde{u}}{\partial u}\right)^{2} \frac{d u}{d t} \frac{d u}{d s}+\frac{\partial \widetilde{u}}{\partial u} \frac{\partial \widetilde{v}}{\partial u}\left(\frac{d u}{d t} \frac{d v}{d s}+\frac{d u}{d s} \frac{d v}{d t}\right)+\left(\frac{\partial \widetilde{v}}{\partial u}\right)^{2} \frac{d v}{d t} \frac{d v}{d s}, \\
\mu_{\widetilde{F}}=2 \cdot\left(\frac{\partial \widetilde{u}}{\partial u} \frac{\partial \widetilde{v}}{\partial u}\right) \frac{d u}{d t} \frac{d u}{d s}+\left(\frac{\partial \widetilde{u}}{\partial u} \frac{\partial \widetilde{v}}{\partial v}+\frac{\partial \widetilde{u}}{\partial v} \frac{\partial \widetilde{v}}{\partial u}\right)\left(\frac{d u}{d t} \frac{d v}{d s}+\frac{d u}{d s} \frac{d v}{d t}\right)+2 \cdot\left(\frac{\partial \widetilde{u}}{\partial v} \frac{\partial \widetilde{v}}{\partial v}\right) \frac{d v}{d t} \frac{d v}{d s},
\end{gathered}
$$

$\mathrm{e}$

$$
\mu_{\widetilde{G}}=\left(\frac{\partial \widetilde{v}}{\partial u}\right)^{2} \frac{d u}{d t} \frac{d u}{d s}+\frac{\partial \widetilde{v}}{\partial u} \frac{\partial \widetilde{v}}{\partial v}\left(\frac{d u}{d t} \frac{d v}{d s}+\frac{d u}{d s} \frac{d v}{d t}\right)+\left(\frac{\partial \widetilde{v}}{\partial v}\right)^{2} \frac{d v}{d t} \frac{d v}{d s}
$$

Mas,

$$
\begin{aligned}
\mu_{\widetilde{E}} & =\left(\frac{\partial \widetilde{u}}{\partial u}\right)^{2} \frac{d u}{d t} \frac{d u}{d s}+\frac{\partial \widetilde{u}}{\partial u} \frac{\partial \widetilde{u}}{\partial v}\left(\frac{d u}{d t} \frac{d v}{d s}+\frac{d u}{d s} \frac{d v}{d t}\right)+\left(\frac{\partial \widetilde{u}}{\partial v}\right)^{2} \frac{d v}{d t} \frac{d v}{d s} \\
& =\left(\frac{\partial \widetilde{u}}{\partial u} \frac{d u}{d t}\right)\left(\frac{\partial \widetilde{u}}{\partial u} \frac{d u}{d s}\right)+\left(\frac{\partial \widetilde{u}}{\partial u} \frac{d u}{d t}\right)\left(\frac{\partial \widetilde{u}}{\partial v} \frac{d v}{d s}\right)+\left(\frac{\partial \widetilde{u}}{\partial u} \frac{d u}{d s}\right)\left(\frac{\partial \widetilde{u}}{\partial v} \frac{d v}{d t}\right)+\left(\frac{\partial \widetilde{u}}{\partial v} \frac{d v}{d t}\right)\left(\frac{\partial \widetilde{u}}{\partial v} \frac{d v}{d s}\right) \\
& =\left(\frac{\partial \widetilde{u}}{\partial u} \frac{d u}{d t}\right)\left(\frac{\partial \widetilde{u}}{\partial u} \frac{d u}{d s}+\frac{\partial \widetilde{u}}{\partial v} \frac{d v}{d s}\right)+\left(\frac{\partial \widetilde{u}}{\partial v} \frac{d v}{d t}\right)\left(\frac{\partial \widetilde{u}}{\partial u} \frac{d u}{d s}+\frac{\partial \widetilde{u}}{\partial v} \frac{d v}{d s}\right) \\
& =\left(\frac{\partial \widetilde{u}}{\partial u} \frac{d u}{d t}+\frac{\partial \widetilde{u}}{\partial v} \frac{d v}{d t}\right)\left(\frac{\partial \widetilde{u}}{\partial u} \frac{d u}{d s}+\frac{\partial \widetilde{u}}{\partial v} \frac{d v}{d s}\right) \\
& =\frac{d \widetilde{u}}{d t} \frac{d \widetilde{v}}{d s},
\end{aligned}
$$




$$
\begin{aligned}
\mu_{\widetilde{F}} & =2 \cdot\left(\frac{\partial \widetilde{u}}{\partial u} \frac{\partial \widetilde{v}}{\partial u}\right) \frac{d u}{d t} \frac{d u}{d s}+\left(\frac{\partial \widetilde{u}}{\partial u} \frac{\partial \widetilde{v}}{\partial v}+\frac{\partial \widetilde{u}}{\partial v} \frac{\partial \widetilde{v}}{\partial u}\right)\left(\frac{d u}{d t} \frac{d v}{d s}+\frac{d u}{d s} \frac{d v}{d t}\right)+2 \cdot\left(\frac{\partial \widetilde{u}}{\partial v} \frac{\partial \widetilde{v}}{\partial v}\right) \frac{d v}{d t} \frac{d v}{d s} \\
& =\left(\frac{\partial \widetilde{u}}{\partial u} \frac{d u}{d t}\right)\left(\frac{\partial \widetilde{v}}{\partial u} \frac{d u}{d s}\right)+\left(\frac{\partial \widetilde{v}}{\partial u} \frac{d u}{d s}\right)\left(\frac{\partial \widetilde{u}}{\partial u} \frac{d u}{d t}\right)+\left(\frac{\partial \widetilde{u}}{\partial u} \frac{d u}{d t}\right)\left(\frac{\partial \widetilde{v}}{\partial v} \frac{d v}{d s}\right)+\left(\frac{\partial \widetilde{u}}{\partial u} \frac{d u}{d s}\right)\left(\frac{\partial \widetilde{v}}{\partial v} \frac{d v}{d t}\right) \\
& +\left(\frac{\partial \widetilde{u}}{\partial v} \frac{d v}{d s}\right)\left(\frac{\partial \widetilde{v}}{\partial u} \frac{d u}{d t}\right)+\left(\frac{\partial \widetilde{u}}{\partial v} \frac{d v}{d t}\right)\left(\frac{\partial \widetilde{v}}{\partial u} \frac{d u}{d s}\right)+\left(\frac{\partial \widetilde{u}}{\partial v} \frac{d v}{d t}\right)\left(\frac{\partial \widetilde{v}}{\partial v} \frac{d v}{d s}\right)+\left(\frac{\partial \widetilde{v}}{\partial v} \frac{d v}{d t}\right)\left(\frac{\partial \widetilde{u}}{\partial v} \frac{d v}{d s}\right) \\
& =\left(\frac{\partial \widetilde{u}}{\partial u} \frac{d u}{d t}\right)\left(\frac{\partial \widetilde{v}}{\partial u} \frac{d u}{d s}+\frac{\partial \widetilde{v}}{\partial v} \frac{d v}{d s}\right)+\left(\frac{\partial \widetilde{u}}{\partial v} \frac{d v}{d t}\right)\left(\frac{\partial \widetilde{v}}{\partial u} \frac{d u}{d s}+\frac{\partial \widetilde{v}}{\partial v} \frac{d v}{d s}\right) \\
& =\left(\frac{\partial \widetilde{u}}{\partial u} \frac{d u}{d s}\right)\left(\frac{\partial \widetilde{v}}{\partial u} \frac{d u}{d t}+\frac{\partial \widetilde{v}}{\partial v} \frac{d v}{d t}\right)+\left(\frac{\partial \widetilde{u}}{\partial v} \frac{d v}{d s}\right)\left(\frac{\partial \widetilde{v}}{\partial u} \frac{d u}{d t}+\frac{\partial \widetilde{v}}{\partial v} \frac{d v}{d t}\right) \\
& =\left(\frac{\partial \widetilde{u}}{\partial u} \frac{d u}{d t}+\frac{\partial \widetilde{u}}{\partial v} \frac{d v}{d t}\right)\left(\frac{\partial \widetilde{v}}{\partial u} \frac{d u}{d s}+\frac{\partial \widetilde{v}}{\partial v} \frac{d v}{d s}\right)+\left(\frac{\partial \widetilde{u}}{\partial u} \frac{d u}{d s}+\frac{\partial \widetilde{u}}{\partial v} \frac{d v}{d s}\right)\left(\frac{\partial \widetilde{v}}{\partial u} \frac{d u}{d t}+\frac{\partial \widetilde{v}}{\partial v} \frac{d v}{d t}\right) \\
& =\frac{d \widetilde{u}}{d t} \frac{d \widetilde{v}}{d s}+\frac{d \widetilde{u}}{d s} \frac{d \widetilde{v}}{d t}
\end{aligned}
$$

e

$$
\begin{aligned}
\mu_{\widetilde{G}} & =\left(\frac{\partial \widetilde{v}}{\partial u}\right)^{2} \frac{d u}{d t} \frac{d u}{d s}+\frac{\partial \widetilde{v}}{\partial u} \frac{\partial \widetilde{v}}{\partial v}\left(\frac{d u}{d t} \frac{d v}{d s}+\frac{d u}{d s} \frac{d v}{d t}\right)+\left(\frac{\partial \widetilde{v}}{\partial v}\right)^{2} \frac{d v}{d t} \frac{d v}{d s} \\
& =\left(\frac{\partial \widetilde{v}}{\partial u} \frac{d u}{d t}\right)\left(\frac{\partial \widetilde{v}}{\partial u} \frac{d u}{d s}\right)+\left(\frac{\partial \widetilde{v}}{\partial u} \frac{d u}{d t}\right)\left(\frac{\partial \widetilde{v}}{\partial v} \frac{d v}{d s}\right)+\left(\frac{\partial \widetilde{v}}{\partial u} \frac{d u}{d s}\right)\left(\frac{\partial \widetilde{v}}{\partial v} \frac{d v}{d t}\right)+\left(\frac{\partial \widetilde{v}}{\partial v} \frac{d v}{d t}\right)\left(\frac{\partial \widetilde{v}}{\partial v} \frac{d v}{d s}\right) \\
& =\left(\frac{\partial \widetilde{v}}{\partial u} \frac{d u}{d t}\right)\left(\frac{\partial \widetilde{v}}{\partial u} \frac{d u}{d s}+\frac{\partial \widetilde{v}}{\partial v} \frac{d v}{d s}\right)+\left(\frac{\partial v}{\partial v} \frac{d v}{d t}\right)\left(\frac{\partial \widetilde{v}}{\partial u} \frac{d u}{d s}+\frac{\partial \widetilde{v}}{\partial v} \frac{d v}{d s}\right) \\
& =\left(\frac{\partial \widetilde{v}}{\partial u} \frac{d u}{d t}+\frac{\partial \widetilde{v}}{\partial v} \frac{d v}{d t}\right)\left(\frac{\partial \widetilde{v}}{\partial u} \frac{d u}{d s}+\frac{\partial \widetilde{v}}{\partial v} \frac{d v}{d s}\right) \\
& =\frac{d \widetilde{v}}{d t} \frac{d \widetilde{v}}{d s} .
\end{aligned}
$$

Então,

$$
E \frac{d u}{d t} \frac{d u}{d s}+F\left(\frac{d u}{d t} \frac{d v}{d s}+\frac{d u}{d s} \frac{d v}{d t}\right)+G \frac{d v}{d t} \frac{d v}{d s}=\widetilde{E} \frac{d \widetilde{u}}{d t} \frac{d \widetilde{u}}{d s}+\widetilde{F}\left(\frac{d \widetilde{u}}{d t} \frac{d \widetilde{v}}{d s}+\frac{d \widetilde{u}}{d s} \frac{d \widetilde{v}}{d t}\right)+\widetilde{G} \frac{d \widetilde{v}}{d t} \frac{d \widetilde{v}}{d s}
$$

Da prova da Preposição B.2, concluímos que

$$
\left(\begin{array}{ll}
d u & d v
\end{array}\right)\left(\begin{array}{cc}
E & F \\
F & G
\end{array}\right)\left(\begin{array}{l}
d u \\
d v
\end{array}\right)=\left(\begin{array}{cc}
d \widetilde{u} & d \widetilde{v}
\end{array}\right)\left(\begin{array}{cc}
\widetilde{E} & \widetilde{F} \\
\widetilde{F} & \widetilde{G}
\end{array}\right)\left(\begin{array}{c}
d \widetilde{u} \\
d \widetilde{v}
\end{array}\right) .
$$

Então,

$$
E\left(\frac{d u}{d t}\right)^{2}+2 F \frac{d u}{d t} \frac{d v}{d t}+G\left(\frac{d v}{d t}\right)^{2}=\widetilde{E}\left(\frac{d \widetilde{u}}{d t}\right)^{2}+2 \widetilde{F} \frac{d \widetilde{u}}{d t} \frac{d \widetilde{v}}{d t}+\widetilde{G}\left(\frac{d \widetilde{v}}{d t}\right)^{2}
$$

e

$$
E\left(\frac{d u}{d s}\right)^{2}+2 F \frac{d u}{d s} \frac{d v}{d s}+G\left(\frac{d v}{d s}\right)^{2}=\widetilde{E}\left(\frac{d \widetilde{u}}{d s}\right)^{2}+2 \widetilde{F} \frac{d \widetilde{u}}{d s} \frac{d \widetilde{v}}{d s}+\widetilde{G}\left(\frac{d \widetilde{v}}{d s}\right)^{2}
$$

o que significa que a equação (C.1) é igual a

$$
\theta=\arccos \left(\frac{\widetilde{E} \frac{d \widetilde{u}}{d t} \frac{d \widetilde{v}}{d s}+\widetilde{F}\left(\frac{d \widetilde{u}}{d t} \frac{d \widetilde{u}}{d s}+\frac{d \widetilde{u}}{d s} \frac{d \widetilde{v}}{d t}\right)+\widetilde{G} \frac{d \widetilde{v}}{d t} \frac{d \widetilde{v}}{d s}}{\left(\widetilde{E}\left(\frac{d \widetilde{u}}{d t}\right)^{2}+2 \widetilde{F} \frac{d \widetilde{u}}{d t} \frac{d \widetilde{v}}{d t}+\widetilde{G}\left(\frac{d \widetilde{v}}{d t}\right)^{2}\right)^{\frac{1}{2}}\left(\widetilde{E}\left(\frac{d \widetilde{u}}{d s}\right)^{2}+2 \widetilde{F} \frac{d \widetilde{u}}{d s} \frac{d \widetilde{v}}{d s}+\widetilde{G}\left(\frac{d \widetilde{v}}{d s}\right)^{2}\right)^{\frac{1}{2}}}\right) .
$$


Proposição B.4. A área de uma região de uma superfície uma não é mudada por uma reparametrização.

Prova: Sejam $S$ uma superfície, $X: U \rightarrow X(U) \subseteq S$ uma parametrização de $S$ definida em um subconjunto aberto $U$ de $\mathbb{E}^{2}$. Seja $D$ uma região do plano com $D \subset U$. Suponha que $Y: W \rightarrow Y(W) \subseteq S$ é outra parametrização de $S$ satisfazendo $X(D) \subset(X(U) \cap Y(W))$ não vazio. Podemos definir um difeomorfismo $h: X^{-1} \circ Y: Y^{-1}(\Omega) \rightarrow X^{-1}(\Omega)$. Então, se $h(\widetilde{u}, \widetilde{v})=(u, v)$, temos

$$
Y(\widetilde{u}, \widetilde{v})=X(u, v)
$$

Seja $\widetilde{D}$ um subconjunto de $W$ tal que $h(\widetilde{D})=D$. Então $h$ é um difiomorfismo, e $\widetilde{D}$ é uma região do plano com $\widetilde{D} \subset Y^{-1}(\Omega)$. Além disso, como $Y(\widetilde{u}, \widetilde{v})=(X \circ h)(\widetilde{u}, \widetilde{v})$ para todo $(\widetilde{u}, \widetilde{v}) \in Y^{-1}(\Omega)$, temos que

$$
(d Y)_{(\widetilde{u}, \widetilde{v})}=(d X)_{(h(\widetilde{u}, \widetilde{v})} \circ(d h)_{(\widetilde{u}, \widetilde{v})}=(d X)_{(u, v)} \circ(d h)_{(\widetilde{u}, \widetilde{v})} .
$$

Então,

$$
Y_{\widetilde{u}}=\frac{\partial u}{\partial \widetilde{u}} X_{u}+\frac{\partial v}{\partial \widetilde{u}} X_{v} \quad \text { e } \quad Y_{\widetilde{v}}=\frac{\partial u}{\partial \widetilde{v}} X_{u}+\frac{\partial v}{\partial \widetilde{v}} X_{v}
$$

onde $u(\widetilde{u}, \widetilde{v})$ e $v(\widetilde{u}, \widetilde{v})$ são componentes da função $h$. Usando as igualdades acima, temos que

$$
Y_{\widetilde{u}} \times Y_{\widetilde{v}}=\left(\frac{\partial u}{\partial \widetilde{u}} \frac{\partial v}{\partial \widetilde{v}}-\frac{\partial u}{\partial \widetilde{v}} \frac{\partial v}{\partial \widetilde{u}}\right)\left(X_{u} \times X_{v}\right)=\operatorname{det}(J(h)(\widetilde{u}, \widetilde{v})) \cdot\left(X_{u} \times X_{v}\right),
$$

onde $\operatorname{det}(J(h)(\widetilde{u}, \widetilde{v}))$ é o determinate de $J(h)(\widetilde{u}, \widetilde{v})$, que é a matriz Jacobiana de $d h(\widetilde{u}, \widetilde{v})$. Por isso, usando o Teorema de mudança de variáveis para integrais duplas,

$$
\begin{aligned}
\int_{\widetilde{D}}\left\|Y_{\widetilde{u}} \times Y_{\widetilde{v}}\right\| d \widetilde{u} d \widetilde{v} & =\int_{\widetilde{D}}|\operatorname{det}(J(h)(\widetilde{u}, \widetilde{v}))| \cdot\left\|X_{u} \times X_{v}\right\| d \widetilde{u} d \widetilde{v} \\
& =\int_{h(\widetilde{D})}\left\|X_{u} \times X_{v}\right\| d u d v \\
& =\int_{D}\left\|X_{u} \times X_{v}\right\| d u d v .
\end{aligned}
$$

Teorema B.3. Sejam $S_{1}$ e $S_{2}$ duas superfícies. Um difeomorfismo $f: S_{1} \rightarrow S_{2}$ é conformal se, e somente se, existe uma função $\lambda: S \rightarrow \mathbb{R}$ tal que, para cada $p \in S_{1}$ e para quaisquer dois vetores $v, w \in T_{p} S_{1}$, temos

$$
\left\langle(d f)_{p}(v),(d f)_{p}(w)\right\rangle_{f(p)}=\lambda(p) \cdot\langle v, w\rangle_{p}
$$


Prova: Sejam $\alpha: I \rightarrow S_{1}$ e $\widetilde{\alpha}: I \rightarrow S_{1}$ duas curvas regulares que se intersectam no ponto $p$ em $S_{1}$, onde podemos supor que $p=\alpha(t)=\widetilde{\alpha}(t)$, para $t=0$. O ângulo $\phi_{1}$ definido por $\alpha$ e $\widetilde{\alpha}$ em $p$ é dado por

$$
\cos \left(\phi_{1}\right)=\frac{\left\langle\alpha^{\prime}(0), \widetilde{\alpha}^{\prime}(0)\right\rangle_{p}}{\left\|\alpha^{\prime}(0)\right\|_{p} \cdot\left\|\widetilde{\alpha}^{\prime}(0)\right\|_{p}} .
$$

Como $f$ é um difeomorfismo, $f$ leva $\alpha$ e $\widetilde{\alpha}$ sobre as curvas regulares $\beta=f \circ \alpha: I \rightarrow S_{2}$ e $\widetilde{\beta}=f \circ \widetilde{\alpha}: I \rightarrow S_{2}$, com intersecção em $f(p)=f(\alpha(0))=\beta(0)=\widetilde{\beta}(0)$ e define um ângulo $\phi_{2}$, dado por

$$
\cos \left(\phi_{2}\right)=\frac{\left\langle\beta^{\prime}(0), \widetilde{\beta}^{\prime}(0)\right\rangle_{f(p)}}{\left\|\beta^{\prime}(0)\right\|_{f(p)} \cdot\left\|\widetilde{\beta}^{\prime}(0)\right\|_{f(p)}}
$$

Mas, $\beta^{\prime}(0)=(f \circ \alpha)^{\prime}(0)=\left((d f)_{p} \circ \alpha^{\prime}\right)(0)$ e $\widetilde{\beta}^{\prime}(0)=(f \circ \widetilde{\alpha})^{\prime}(0)=\left((d f)_{p} \circ \widetilde{\alpha}^{\prime}\right)(0)$. Então, podemos escrever $\cos \left(\phi_{2}\right)$ como

$$
\cos \left(\phi_{2}\right)=\frac{\left\langle\left((d f)_{p} \circ \alpha^{\prime}\right)(0),\left((d f)_{p} \circ \widetilde{\alpha}^{\prime}\right)(0)\right\rangle_{f(p)}}{\left\|\left((d f)_{p} \circ \alpha^{\prime}\right)(0)\right\|_{f(p)} \cdot\left\|\left((d f)_{p} \circ \widetilde{\alpha}^{\prime}\right)(0)\right\|_{f(p)}} .
$$

$\operatorname{Se}\left\langle(d f)_{p}(v),(d f)_{p}(w)\right\rangle_{f(p)}=\lambda(p) \cdot\langle v, w\rangle_{p}$, para todo $v, w \in T_{p} S_{1}$, então, se $v=\alpha^{\prime}(0)$ e $w=\widetilde{\alpha}^{\prime}(0)$, temos

$$
\cos \left(\phi_{2}\right)=\frac{\lambda(p) \cdot\left\langle\alpha^{\prime}(0), \widetilde{\alpha}^{\prime}(0)\right\rangle_{p}}{\lambda(p) \cdot\left\|\alpha^{\prime}(0)\right\|_{p} \cdot\left\|\widetilde{\alpha}^{\prime}(0)\right\|_{p}}=\frac{\left\langle\alpha^{\prime}(0), \widetilde{\alpha}^{\prime}(0)\right\rangle_{p}}{\left\|\alpha^{\prime}(0)\right\|_{p} \cdot\left\|\widetilde{\alpha}^{\prime}(0)\right\|_{p}}=\cos \left(\phi_{1}\right) .
$$

De fato, $f$ é conformal.

Reciprocamente, supomos que $f$ é conformal. Como todo vetor tangente a $S_{1}$ é um vetor tangente a uma curva em $S_{1}$, a seguinte igualdade deve garantir, para qualquer ponto $p \in S_{1}$ e para quaisquer dois vetores $v, w \in T_{p} S$,

$$
\frac{\langle v, w\rangle_{p}}{\|v\|_{p} \cdot\|w\|_{p}}=\frac{\left\langle(d f)_{p}(v),(d f)_{p}(w)\right\rangle_{f(p)}}{\left\|(d f)_{p}(v)\right\|_{f(p)} \cdot\left\|(d f)_{p}(w)\right\|_{f(p)}}
$$

Seja $\left\{v_{1}, v_{2}\right\}$ uma base ortonormal de $T_{p} S_{1}$ com respeito a $\langle,\rangle_{p}$. Denotamos $\left\langle(d f)_{p}(v),(d f)_{p}(w)\right\rangle_{f(p)}$ por $f^{*}\langle v, w\rangle_{f(p)}$, e

$$
\lambda=f^{*}\left\langle v_{1}, v_{1}\right\rangle_{f(p)}, \quad \mu=f^{*}\left\langle v_{1}, v_{2}\right\rangle_{f(p)} \quad \text { e } \quad \nu=f^{*}\left\langle v_{2}, v_{2}\right\rangle_{f(p)} .
$$

Se tomamos $v=v_{1}$ e $w=\cos (\phi) \cdot v_{1}+\sin (\phi) \cdot v_{2}$, então

$$
\begin{gathered}
\langle v, w\rangle_{p}=\cos (\phi) \cdot\left\langle v_{1}, v_{1}\right\rangle_{p}+\sin (\phi) \cdot\left\langle v_{1}, v_{2}\right\rangle_{p}=\cos (\phi) \cdot 1+\sin (\phi) \cdot 0, \\
\|v\|_{p}=\left(\left\langle v_{1}, v_{1}\right\rangle_{p}\right)^{\frac{1}{2}}=1
\end{gathered}
$$


e

$$
\begin{aligned}
\|w\|_{p} & =\left(\left\langle\cos (\phi) \cdot v_{1}+\sin (\phi) \cdot v_{2}, \cos (\phi) \cdot v_{1}+\sin (\phi) \cdot v_{2}\right\rangle_{p}\right)^{\frac{1}{2}} \\
& =\left(\cos (\phi)^{2} \cdot\left\langle v_{1}, v_{1}\right\rangle_{p}+2 \cdot \cos (\phi) \cdot \sin (\phi) \cdot\left\langle v_{1}, v_{2}\right\rangle_{p}+\sin (\phi)^{2} \cdot\left\langle v_{2}, v_{2}\right\rangle_{p}\right)^{\frac{1}{2}} \\
& =\left(\cos (\phi)^{2} \cdot 1+2 \cdot \cos (\phi) \cdot \sin (\phi) \cdot 0+\sin (\phi)^{2} \cdot 1\right)^{\frac{1}{2}} \\
& =\left(\cos (\phi)^{2}+\sin (\phi)^{2}\right)^{\frac{1}{2}} \\
& =1
\end{aligned}
$$

Assim,

$$
\frac{\langle v, w\rangle_{p}}{\|v\|_{p} \cdot\|w\|_{p}}=\frac{\cos (\phi)}{1 \cdot 1}=\cos (\phi)
$$

Por sua vez,

$$
\begin{gathered}
f^{*}\langle v, w\rangle_{f(p)}=\cos (\phi) \cdot f^{*}\left\langle v_{1}, v_{1}\right\rangle_{f(p)}+\sin (\phi) \cdot f^{*}\left\langle v_{1}, v_{2}\right\rangle_{f(p)}=\cos (\phi) \cdot \lambda+\sin (\phi) \cdot \mu \\
\left\|(d f)_{p}(v)\right\|_{f(p)}=\left(\left\langle(d f)_{p}\left(v_{1}\right),(d f)_{p}\left(v_{1}\right)\right\rangle_{f(p)}\right)^{\frac{1}{2}}=\left(f^{*}\left\langle v_{1}, v_{1}\right\rangle_{f(p)}\right)^{\frac{1}{2}}=\sqrt{\lambda}
\end{gathered}
$$

e

$$
\begin{aligned}
\left\|(d f)_{p}(w)\right\|_{f(p)} & =\left(\left\langle(d f)_{p}(w),(d f)_{p}(w)\right\rangle_{f(p)}\right)^{\frac{1}{2}} \\
& =\left(\left\langle(d f)_{p}\left(\cos (\phi) \cdot v_{1}+\sin (\phi) \cdot v_{2}\right),(d f)_{p}\left(\cos (\phi) \cdot v_{1}+\sin (\phi) \cdot v_{2}\right)\right\rangle_{f(p)}\right)^{\frac{1}{2}} \\
& =\left(\left\langle(d f)_{p}\left(\cos (\phi) \cdot v_{1}\right)+(d f)_{p}\left(\sin (\phi) \cdot v_{2}\right),(d f)_{p}\left(\cos (\phi) \cdot v_{1}\right)+\right.\right. \\
& \left.\left.+(d f)_{p}\left(\sin (\phi) \cdot v_{2}\right)\right\rangle_{f(p)}\right)^{\frac{1}{2}} \\
& =\left(\cos (\phi)^{2} \cdot\left\langle(d f)_{p}\left(v_{1}\right),(d f)_{p}\left(v_{1}\right)\right\rangle_{f(p)}+2 \cdot \cos (\phi) \cdot \sin (\phi)\left\langle(d f)_{p}\left(v_{1}\right),\right.\right. \\
& \left.\left.(d f)_{p}\left(v_{2}\right)\right\rangle_{f(p)}+\sin (\phi)^{2} \cdot\left\langle(d f)_{p}\left(v_{2}\right),(d f)_{p}\left(v_{2}\right)\right\rangle_{f(p)}\right)^{\frac{1}{2}} \\
& =\left(\cos (\phi)^{2} \cdot f^{*}\left\langle v_{1}, v_{1}\right\rangle_{f(p)}+2 \cdot \cos (\phi) \cdot \sin (\phi) \cdot f^{*}\left\langle v_{1}, v_{2}\right\rangle_{f(p)}+\right. \\
& \left.+\sin (\phi)^{2} \cdot f^{*}\left\langle v_{2}, v_{2}\right\rangle_{f(p)}\right)^{\frac{1}{2}} \\
& =\left(\cos (\phi)^{2} \cdot \lambda+2 \cdot \cos (\phi) \cdot \sin (\phi) \cdot \mu+\sin (\phi)^{2} \cdot \nu\right)^{\frac{1}{2}} .
\end{aligned}
$$

\section{Então,}

$$
\frac{\left\langle(d f)_{p}(v),(d f)_{p}(w)\right\rangle_{f(p)}}{\left\|(d f)_{p}(v)\right\|_{f(p)} \cdot\left\|(d f)_{p}(w)\right\|_{f(p)}}=\frac{\cos (\phi) \cdot \lambda+\sin (\phi) \cdot \mu}{\sqrt{\lambda \cdot\left(\cos (\phi)^{2} \cdot \lambda+2 \cdot \cos (\phi) \cdot \sin (\phi) \cdot \mu+\sin (\phi)^{2} \cdot \nu\right)}} .
$$

\section{Como}

$$
\frac{\langle v, w\rangle_{p}}{\|v\|_{p} \cdot\|w\|_{p}}=\frac{\left\langle(d f)_{p}(v),(d f)_{p}(w)\right\rangle_{f(p)}}{\left\|(d f)_{p}(v)\right\|_{f(p)} \cdot\left\|(d f)_{p}(w)\right\|_{f(p)}}
$$


temos

$$
\cos (\phi)=\frac{\cos (\phi) \cdot \lambda+\sin (\phi) \cdot \mu}{\sqrt{\lambda \cdot\left(\cos (\phi)^{2} \cdot \lambda+2 \cdot \cos (\phi) \cdot \sin (\phi) \cdot \mu+\sin (\phi)^{2} \cdot \nu\right)}} .
$$

Tomando $\phi=\frac{\pi}{2}$, temos $\mu=0$. Então,

$$
\cos (\phi)=\frac{\cos (\phi) \cdot \lambda}{\sqrt{\lambda \cdot\left(\cos (\phi)^{2} \cdot \lambda+\sin (\phi)^{2} \cdot \nu\right)}},
$$

o que implica que $\cos (\phi)^{2} \cdot \lambda+\sin (\phi)^{2} \cdot \nu=\lambda$, para todo $\phi \in \mathbb{R}$. Mas, então, temos $\nu=\lambda$.

Deste modo, se

$$
v=a \cdot v_{1}+b \cdot v_{2} \quad \text { e } \quad w=c \cdot v_{1}+d \cdot v_{2},
$$

para todo $a, b, c, d \in \mathbb{R}$, temos

$$
\begin{aligned}
f^{*}\langle v, w\rangle_{f(p)} & =a \cdot c \cdot f^{*}\left\langle v_{1}, v_{1}\right\rangle_{f(p)}+(a \cdot c+b \cdot d) \cdot f^{*}\left\langle v_{1}, v_{2}\right\rangle_{f(p)}+b \cdot d \cdot f^{*}\left\langle v_{2}, v_{2}\right\rangle_{f(p)} \\
& =\lambda \cdot(a \cdot c+b \cdot d) \\
& =\lambda \cdot\langle v, w\rangle_{p} .
\end{aligned}
$$

Teorema B.4. Sejam $S_{1}$ e $S_{2}$ duas superfícies. Um difeomorfismo $f: S_{1} \rightarrow S_{2}$ é equiareal se, e somente se, para qualquer parametrização $X: U \rightarrow X(U) \subseteq S_{1}$ e para cada $p \in X(U)$, os coeficientes, $E_{1}, F_{1}, G_{1}$ e $E_{2}, F_{2}, G_{2}$ da Primeira Forma Fundamental de $S_{1}$ e $S_{2}$ em p e $f(p)$ com respeito a $X$ e $f \circ X$, respectivamente, satisfaz

$$
E_{1} \cdot G_{1}-F_{1}^{2}=E_{2} \cdot G_{2}-F_{2}^{2}
$$

Prova: Sejam $X: U \rightarrow X(U) \subseteq S_{1}$ uma parametrização em $S_{1}, Y=f \circ X: U \rightarrow(f \circ$ $X)(U) \subseteq S_{2}$ uma parametrização em $S_{2}, D$ toda região em $\mathbb{E}^{2}$ com $D \subset U$. Sejam $E_{1}, F_{1}, G_{1}$ e $E_{2}, F_{2}, G_{2}$ os coeficientes da Primeira Forma Fundamental de $S_{1}$ e $S_{2}$, respectivamente. Se, para cada $p \in X(U), E_{1} \cdot G_{1}-F_{1}^{2}$ e $E_{2} \cdot G_{2}-F_{2}^{2}$ são iguais em $(u, v)=X^{-1}(p)$, então a Proposição B.1 implica que

$$
\begin{aligned}
\int_{D}\left\|X_{u} \times X_{v}\right\| d u d v & =\int_{D}\left(E_{1} \cdot G_{1}-F_{1}^{2}\right)^{\frac{1}{2}} d u d v \\
& =\int_{D}\left(E_{2} \cdot G_{2}-F_{2}^{2}\right)^{\frac{1}{2}} d u d v \\
& =\int_{D}\left\|Y_{u} \times Y_{v}\right\| d u d v .
\end{aligned}
$$

Reciprocamente, se

$$
\int_{D}\left\|X_{u} \times X_{v}\right\| d u d v=\int_{D}\left\|Y_{u} \times Y_{v}\right\| d u d v
$$


para toda região $D \subset U$, então $\left\|X_{u} \times X_{v}\right\|=\left\|Y_{u} \times Y_{v}\right\|$, para cada $(u, v) \in U$. Assim, pela Proposição B.1, temos

$$
\left(E_{1} \cdot G_{1}-F_{1}^{2}\right)^{\frac{1}{2}}=\left(E_{2} \cdot G_{2}-F_{2}^{2}\right)^{\frac{1}{2}} .
$$

Como $E_{1} \cdot G_{1}-F_{1}^{2}>0$ e $E_{2} \cdot G_{2}-F_{2}^{2}>0$, temos que $E_{1} \cdot G_{1}-F_{1}^{2}=E_{2} \cdot G_{2}-F_{2}^{2}$, para cada $(u, v) \in U$.

Teorema B.5. Sejam $S_{1}$ e $S_{2}$ duas superfícies. Um mapa suave $f: S_{1} \rightarrow S_{2}$ é uma isometria local se, e somente se,

$$
\langle v, w\rangle_{p}=\left\langle(d f)_{p}(v),(d f)_{p}(w)\right\rangle_{f(p)},
$$

para todo ponto p em $S_{1}$ e para todo par de vetores $v$ e $w$ em $T_{p} S_{1}$.

Prova: Se $\alpha$ é uma curva em $S_{1}$, então o comprimento do segmento de $\alpha$ em $\alpha\left(t_{0}\right)$ e $\alpha\left(t_{1}\right)$ é dado por

$$
\int_{t_{0}}^{t_{1}}\left(\left\langle\alpha^{\prime}(t), \alpha^{\prime}(t)\right\rangle_{\alpha(t)}\right)^{\frac{1}{2}} d t
$$

Se $\beta=f \circ \alpha$ é a imagem de $\alpha$ em $S_{2}$ inferior a $f$, então o comprimento correspondente ao segmento de $\beta$ é

$$
\int_{t_{0}}^{t_{1}}\left(\left\langle\beta^{\prime}(t), \beta^{\prime}(t)\right\rangle_{\beta(t)}\right)^{\frac{1}{2}} d t=\int_{t_{0}}^{t_{1}}\left(\left\langle(d f)_{\alpha(t)}\left(\alpha^{\prime}(t)\right),(d f)_{\alpha(t)}\left(\alpha^{\prime}(t)\right)\right\rangle_{\beta(t)}\right)^{\frac{1}{2}} .
$$

$\mathrm{Se}$

$$
\langle v, w\rangle_{p}=\left\langle(d f)_{p}(v),(d f)_{p}(w)\right\rangle_{f(p)},
$$

para todo $p$ em $S_{1}$ e para todo $v$ e $w$ em $T_{p} S_{1}$, então, escrevendo $p=\alpha(t)$ e $v=w=\alpha^{\prime}(t)$, temos

$$
\int_{t_{0}}^{t_{1}}\left(\left\langle(d f)_{\alpha(t)}\left(\alpha^{\prime}(t)\right),(d f)_{\alpha(t)}\left(\alpha^{\prime}(t)\right)\right\rangle_{\beta(t)}\right)^{\frac{1}{2}}=\int_{t_{0}}^{t_{1}}\left(\left\langle\alpha^{\prime}(t), \alpha^{\prime}(t)\right\rangle_{\alpha(t)}\right)^{\frac{1}{2}} d t
$$

o que significa que

$$
\int_{t_{0}}^{t_{1}}\left(\left\langle\beta^{\prime}(t), \beta^{\prime}(t)\right\rangle_{\beta(t)}\right)^{\frac{1}{2}} d t=\int_{t_{0}}^{t_{1}}\left(\left\langle\alpha^{\prime}(t), \alpha^{\prime}(t)\right\rangle_{\alpha(t)}\right)^{\frac{1}{2}} d t
$$

e, deste modo, $\alpha$ e $\beta=f \circ \alpha$ tem o mesmo tamanho.

Reciprocamente, se $f: S_{1} \rightarrow S_{2}$ é uma isometria local, então

$$
\int_{t_{0}}^{t_{1}}\left(\left\langle\beta^{\prime}(t), \beta^{\prime}(t)\right\rangle_{\beta(t)}\right)^{\frac{1}{2}} d t=\int_{t_{0}}^{t_{1}}\left(\left\langle\alpha^{\prime}(t), \alpha^{\prime}(t)\right\rangle_{\alpha(t)}\right)^{\frac{1}{2}} d t
$$


para toda curva $\alpha$ em $S_{1}$ e toda curva $\beta=f \circ \alpha$ em $S_{2}$. Então, os integrantes devem ser o mesmo, isto é,

$$
\left\langle\beta^{\prime}(t), \beta^{\prime}(t)\right\rangle_{\beta(t)}=\left\langle\alpha^{\prime}(t), \alpha^{\prime}(t)\right\rangle_{\alpha(t)} .
$$

Mas, como todo vetor tangente $v$ a $S_{1}$ é um vetor tangente de alguma curva em $S_{1}$, temos

$$
\langle v, v\rangle_{p}=\left\langle(d f)_{p}(v),(d f)_{p}(v)\right\rangle_{f(p)},
$$

para todo ponto $p$ em $S_{1}$ e todo vetor $v$ em $T_{p} S_{1}$. Finalmente, como $\langle\cdot, \cdot\rangle_{p}$ e $\left\langle(d f)_{p}(\cdot),(d f)_{p}(\cdot)\right\rangle_{f(p)}$ são formas bi-linear simétrica, temos que $\langle v+w, v+w\rangle_{p}$ e $\left\langle(d f)_{p}(v+w),(d f)_{p}(v+w)\right\rangle_{f(p)}$ são iguais a

$$
\langle v, v\rangle_{p}+2 \cdot\langle v, w\rangle_{p}+\langle w, w\rangle_{p}
$$

e

$$
\left\langle(d f)_{p}(v),(d f)_{p}(v)\right\rangle_{f(p)}+2 \cdot\left\langle(d f)_{p}(v),(d f)_{p}(w)\right\rangle_{f(p)}+\left\langle(d f)_{p}(w),(d f)_{p}(w)\right\rangle_{f(p)},
$$

respectivamente, para quaisquer dois vetores $v$ e $w$ em $T_{p} S_{1}$. Mas, por hipótese, sabemos que

$$
\begin{aligned}
\langle v, v\rangle_{p} & =\left\langle(d f)_{p}(v),(d f)_{p}(v)\right\rangle_{f(p)}, \\
\langle w, w\rangle_{p} & =\left\langle(d f)_{p}(w),(d f)_{p}(w)\right\rangle_{f(p)}
\end{aligned}
$$

e

$$
\langle v+w, v+w\rangle_{p}=\left\langle(d f)_{p}(v+w),(d f)_{p}(v+w)\right\rangle_{f(p)}
$$

Portanto,

$$
2 \cdot\langle v, w\rangle_{p}=2 \cdot\left\langle(d f)_{p}(v),(d f)_{p}(w)\right\rangle_{f(p)} \Rightarrow\langle v, w\rangle_{p}=\left\langle(d f)_{p}(v),(d f)_{p}(w)\right\rangle_{f(p)} .
$$

Teorema B.1. Sejam $S_{1}$ e $S_{2}$ duas superfícies. Então, um mapa suave $f: S_{1} \rightarrow S_{2}$ é uma isometria local se, e somente se, para qualquer parametrização $X: U \rightarrow X(U) \subseteq S_{1}$ e para todo ponto $p \in X(U)$, a Primeira Forma Fundamental de $S_{1}$ e $S_{2}$ em p e $f(p)$ com respeito a $X$ e $f \circ X$, respectivamente, são o mesmo.

Prova: Sejam $X: U \rightarrow X(U) \subseteq S_{1}$ uma parametrização de $S_{1}$, e $Y=f \circ X: U \rightarrow$ $(f \circ X)(U) \subseteq S_{2}$ uma parametrização de $S_{2}$. Denotamos por $E_{1}, F_{1}, G_{1}$ e $E_{2}, F_{2}, G_{2}$ os coeficientes da Primeira Forma Fundamental de $S_{1}$ e $S_{2}$ com respeito a $X$ e $Y$, respectivamente. Por definição, temos que

$$
E_{1}(u, v)=\left\langle\frac{\partial X}{\partial u}(u, v), \frac{\partial X}{\partial u}(u, v)\right\rangle_{p} \quad \text { e } \quad E_{2}(u, v)=\left\langle\frac{\partial Y}{\partial u}(u, v), \frac{\partial Y}{\partial u}(u, v)\right\rangle_{f(p)}
$$




$$
F_{1}(u, v)=\left\langle\frac{\partial X}{\partial u}(u, v), \frac{\partial X}{\partial v}(u, v)\right\rangle_{p} \quad \text { e } \quad F_{2}(u, v)=\left\langle\frac{\partial Y}{\partial u}(u, v), \frac{\partial Y}{\partial v}(u, v)\right\rangle_{f(p)}
$$

e

$$
G_{1}(u, v)=\left\langle\frac{\partial X}{\partial v}(u, v), \frac{\partial X}{\partial v}(u, v)\right\rangle_{p} \quad \text { e } \quad G_{2}(u, v)=\left\langle\frac{\partial Y}{\partial v}(u, v), \frac{\partial Y}{\partial v}(u, v)\right\rangle_{f(p)}
$$

$\operatorname{com} p=X_{1}(u, v)$, para todo $(u, v) \in U$. Como $Y=f \circ X$, temos

$$
\begin{aligned}
\left\langle\frac{\partial Y}{\partial u}(u, v), \frac{\partial Y}{\partial u}(u, v)\right\rangle_{f(p)} & =\left\langle(d f)_{p}\left(\frac{\partial X}{\partial u}\right)(u, v),(d f)_{p}\left(\frac{\partial X}{\partial u}\right)(u, v)\right\rangle_{f(p)} \\
\left\langle\frac{\partial Y}{\partial u}(u, v), \frac{\partial Y}{\partial v}(u, v)\right\rangle_{f(p)} & =\left\langle(d f)_{p}\left(\frac{\partial X}{\partial u}\right)(u, v),(d f)_{p}\left(\frac{\partial X}{\partial v}\right)(u, v)\right\rangle_{f(p)}
\end{aligned}
$$

e

$$
\left\langle\frac{\partial Y}{\partial v}(u, v), \frac{\partial Y}{\partial v}(u, v)\right\rangle_{f(p)}=\left\langle(d f)_{p}\left(\frac{\partial X}{\partial v}\right)(u, v),(d f)_{p}\left(\frac{\partial X}{\partial v}\right)(u, v)\right\rangle_{f(p)} .
$$

Se $f$ é uma isometria local, então

$$
\langle v, w\rangle_{p}=\left\langle(d f)_{p}(v),(d f)_{p}(w)\right\rangle_{f(p)}
$$

para todo ponto $p$ em $S_{1}$ e para quaisquer dois vetores, $v$ e $w$ em $T_{p} S_{1}$. Isso significa que

$$
\begin{aligned}
& E_{2}(u, v)=\left\langle\frac{\partial Y}{\partial u}(u, v), \frac{\partial Y}{\partial u}(u, v)\right\rangle_{f(p)}=\left\langle\frac{\partial X}{\partial u}(u, v), \frac{\partial X}{\partial u}(u, v)\right\rangle_{p}=E_{1}(u, v), \\
& F_{2}(u, v)=\left\langle\frac{\partial Y}{\partial u}(u, v), \frac{\partial Y}{\partial v}(u, v)\right\rangle_{f(p)}=\left\langle\frac{\partial X}{\partial u}(u, v), \frac{\partial X}{\partial v}(u, v)\right\rangle_{p}=F_{1}(u, v), \\
& G_{2}(u, v)=\left\langle\frac{\partial Y}{\partial v}(u, v), \frac{\partial Y}{\partial v}(u, v)\right\rangle_{f(p)}=\left\langle\frac{\partial X}{\partial v}(u, v), \frac{\partial X}{\partial v}(u, v)\right\rangle_{p}=G_{1}(u, v),
\end{aligned}
$$

e

e, portanto, as superfícies $S_{1}$ e $S_{2}$ tem a mesma forma fundamental.

Reciprocamente, se as superfícies $S_{1}$ e $S_{2}$ tem a mesma forma fundamental, então as três identidades acima se mantem, o que significa que

$$
\langle v, w\rangle_{p}=\left\langle(d f)_{p}(v),(d f)_{p}(w)\right\rangle_{f(p)}
$$

toda vez que os vetores tangentes $v$ e $w$ são iguais a $\frac{\partial X}{\partial u}$ ou $\frac{\partial X}{\partial v}$. Mas, como estes dois vetores formam uma base de $T_{p} S_{1}$ e, como $\langle\cdot, \cdot\rangle_{p}$ é uma forma bilinear, temos $\langle v, w\rangle_{p}=\left\langle(d f)_{p}(v),(d f)_{p}(w)\right\rangle_{f(p)}$, para todo $v, w \in T_{p} S_{1}$. 


\section{Referências Bibliográficas}

Andreani, R.; Birgin, E. G.; Martínez, J. M.; Schuverdt, M. L. On augmented lagrangian methods with general lower-level constraints. SIAM Journal on Optimization, 18, 1286-1309, 2007.

Andreani, R.; Martínez, J. M.; Schuverdt, M. L. The constant positive linear dependence condition of qi and wei implies the quasinormality constraint qualification. 2004.

Andretta, M.; Birgin, E. G.; Martínez., J. M. Practical active-set euclidian trust-region method with spectral projected gradients for bound-constrained minimization. Optimization, 54, 305325, 2005.

Birgin, E. G.; Castillo, R.; MArtínez, J. Numerical comparison of augmented lagrangian algorithms for nonconvex problems. Computational Optimization and Applications, 31, 31-56, 2005.

Birgin, E. G.; Martínez, J. M. Large-scale active-set box-constrained optimization method with spectral projected gradients. Computational Optimization and Applications, 23, 101-125, 2002.

Birgin, E. G.; Martínez, J. M.; Raydan, M. Nonmonotone spectral projected gradient methods on convex sets. SIAM Journal on Optimization, 10, 1196-1211, 2000.

BLOCH, E. D. A first course in geometric topology and differential geometry. 3. Birkahäuser, 1997.

Bongartz, I.; Conn, A. R.; Gould, N. I. M.; Toint, P. L. Cute: constrained and unconstrained testing environment. ACM Transactions on Mathematical Software, 23, 123 160, 1995.

DO CARMo, M. P. Elementos de geometria diferencial. 2. Rio de Janeiro: Ao Livro Técnico S.A., 1971. 
Do Carmo, M. P. Differential geometry of curves and surfaces. 3. Prentice-Hall, 1976.

Degener, P.; Meseth, J.; Klein, R. An adaptable surface parameterization method. 12th International Meshing Roundtable, 201-213, 2003.

FIELD, D. A. Laplacian smoothing and delaunay triangulations. Communications in Appied Numerical Methods, 4, 709-712, 1988.

FitzPATRICK, P. M. Advanced calculus. 3, 3.1, 3.6, 2 . American Mathematical Society, 2006.

Frey, P. J.; GeOrGe, P.-L. Mesh generation application to finite elements. Hermes Science Publishing, 2000.

GALLIER, J. Geometric methods and applications for computer science and engineering, 38 of TAM 3. 2. New York: Sprinter, 2011.

GRAY, J. On the history of the riemann mapping theorem, 34 4.1. Rendiconti del Circolo Matematico di Palermo, 47-94, 1994.

Gross, J. L.; Tucker, T. W. Topological graph theory. Mineola, NY, USA: Dover Publications, 2001.

Guibas, L.; Stolfi, J. Primitives for the manipulation of general subdivisions and the computation of voronoi diagrams., 4. ACM Transactions on Graphics, 74-123 , 1985.

Hestenes, M. R. Multiplier and gradient methods. Journal of Otimization Theory and Applications, 4, 303-320, 1969.

Lizier, M. A. S.; Siqueira, M.; II, J. D.; Silva, C. T.; Nonato, L. G. Template-based remeshing for image decomposition. IEEE Proceedings of Sibgrapi'10, 1-8, 2010.

LOHNER, R.; MORGAn, K.; ZIENKIEWICZ, O. Adaptive grid refinement for the compressible euler equations. Wiley, 281-297, 1987.

MCGuire, M. The half-edge data structure. Último acesso: 04/01/2012, 2000.

Disponível em http://www.flipcode.com/archives/The_Half-Edge_ Data_structure.shtml

Moré, J. J.; Sorensen, D. C. Computing a trust region step. SIAM Journal on Scientific and Statistical Computing, 4, 553 - 572, 1983.

Powell, M. J. D. A method for nonlinear constraints in minimization problems Academic Press, 283-298, 1969.

PReSSLEY, A. Elementary differential geometry. 3,2 . New York: SUMS. Springer, 2010. 
QI, L.; WEI, Z. On the constant positive linear dependence condition and its application to sqp methods. SIAM Journal on Optimization, 10, 963981, 2000.

RockAfEllar, R. T. The multiplier method of hestenes and powell applied to convex programming. Journal of Otimization Theory and Applications, 12, 555-562, 1973.

SCHNEIDER, P. J.; EBERLY, D. H. Geometric tools for computer graphics. San Francisco, USA: Elsevier Science, 171-189, 2003.

Siqueira, M.; Xu, D.; Gallier, J.; Nonato, L. G.; Morera, D. M.; Velho, L. A new construction of smooth surfaces from triangle meshes using parametric pseudo-manifolds. Journal Computers and Graphics, 33, 331-340, 2009.

TANGO Project: Trustable algorithms for nonlinear general optimization. 2005.

Disponível em http://www.ime.usp.br/ egbirgin/tango/ 